\title{
Dynamics of wave fluctuations in the homogeneous Yang-Mills condensate
}

\author{
Roman Pasechnik, ${ }^{a}$ George Prokhorov ${ }^{b}$ and Grigory Vereshkov ${ }^{b, 1}$ \\ ${ }^{a}$ Theoretical High Energy Physics, Department of Astronomy and Theoretical Physics, \\ Lund University, Sölvegatan 14A, SE 223-62 Lund, Sweden \\ ${ }^{b}$ Research Institute of Physics, Southern Federal University, \\ 344090 Rostov-on-Don, Russian Federation \\ E-mail: Roman.Pasechnik@thep.lu.se, George.Prokhorov@thep.lu.se, \\ gveresh@gmail.com
}

ABSTRACT: In the present work, the Yang-Mills (YM) quantum-wave excitations of the classical homogeneous YM condensate have been studied in quasi-classical approximation. The formalism is initially formulated in the Hamilton gauge and is based upon canonical quantisation in the Heisenberg representation. This canonical framework is then extended and related to YM dynamics in arbitrary gauge and symmetry group containing at least one SU(2) subgroup. Such generic properties of the interacting YM system as excitation of longitudinal wave modes and energy balance between the evolving YM condensate and waves have been established. In order to prove these findings, the canonical quasi-classical YM system "waves + condensate" in the pure simplest $\mathrm{SU}(2)$ gauge theory has been thoroughly analysed numerically in the linear and next-to-linear approximations in the limit of small wave amplitudes. The effective gluon mass dynamically generated by wave self-interactions in the gluon plasma has been derived. A complete set of equations of motion for the YM "condensate + waves" system accounting for second- and third-order interactions between the waves has been obtained. In the next-to-linear approximation in waves we have found that due to interactions between the YM waves and the YM condensate, the latter looses its energy leading to the growth of amplitudes of the YM wave modes. A similar effect has been found in the maximally-supersymmetric $\mathcal{N}=4$ Yang-Mills theory as well as in two-condensate SU(4) model. Possible implications of these findings to Cosmology and gluon plasma physics have been discussed.

Keywords: Nonperturbative Effects, Gauge Symmetry, Solitons Monopoles and Instantons, Integrable Field Theories

ARXIV EPRINT: 1307.5695

\footnotetext{
${ }^{1}$ Also at Institute for Nuclear Research of Russian Academy of Sciences, 117312 Moscow, Russian Federation.
} 


\section{Contents}

1 Introduction $\quad 2$

2 Overview of Yang-Mills theory in the Hamilton gauge 6

2.1 Degenerate Yang-Mills system 6

2.2 Non-degenerate Yang-Mills system: "condensate + waves" 8

3 Yang-Mills theory in an arbitrary gauge $\quad 9$

$\begin{array}{ll}3.1 & \text { Degenerate Yang-Mills system }\end{array}$

3.2 Non-degenerate Yang-Mills system: "condensate + waves" 12

$\begin{array}{lll}3.3 & \text { Non-local gauge transformations } & 13\end{array}$

4 Yang-Mills dynamics in the Hamilton gauge: one condensate model 15

$\begin{array}{lll}4.1 & \text { Free condensate case } & 15\end{array}$

4.2 Condensate and wave dynamics in the linear approximation 16

4.2.1 First-order Yang-Mills equations of motion 16

4.2.2 Longitudinal Yang-Mills wave modes: free vs interacting case $\quad 19$

$\begin{array}{ll}\text { 4.2.3 Evolution of the Yang-Mills wave modes } & 19\end{array}$

4.3 "Back reaction" of the waves to the condensate 21

4.4 Higher-order corrections 24

5 Dynamics of the Yang-Mills plasma $\quad 25$

5.1 Effective gluon mass from wave self-interactions 26

5.2 Plasma equations with second- and third-order wave self-interactions 29

5.3 Equations for condensate and waves with all wave self-interaction terms up $\begin{array}{ll}\text { to } g^{2} \text { order } & 31\end{array}$

6 Condensate and waves in a supersymmetric Yang-Mills theory 32

7 Cosmological evolution of the Yang-Mills condensate 35

$\begin{array}{lll}7.1 & \text { Free condensate case } & 35\end{array}$

7.2 Quasi-free condensate and vacuum polarisation effects 36

7.3 Super-Yang-Mills condensate decay in quasi-linear approximation 38

8 An overview of the two-condensate SU(4) model 39

$\begin{array}{lll}9 & \text { Summary } & 41\end{array}$

A The method of infinitesimal parameter 43

B Canonical quantization of the Yang-Mills wave modes 45 


\section{Introduction}

A consistent dynamical theory of the non-perturbative Yang-Mills (YM) vacuum, responsible e.g. for the spontaneous chiral symmetry breaking and color confinement phenomena in quantum chromodynamics (QCD) [1-4], has not yet been created. This is indeed one of the biggest theoretical challenges of modern quantum field theory which has a large variety of important implications. For example, self-interacting YM fields play an important role in yet poorly known quark-gluon plasma dynamics at high and low temperatures, including the problem of QCD phase transition and QCD dynamics at large distances. The YM fields are expected to play an important role in pre-equilibrium evolution at early times of relativistic heavy-ion collisions when a Bose condensation of gluons could develop [5-7]. Also, the non-Abelian gauge fields dynamics have important applications in early Universe which are being intensively studied in many different aspects, in particular, in the context of the Dark Energy [8-12] and the non-Abelian fields driven inflation ("gaugeflation") $[13,14]$. Moreover, the modern Dark Energy can be in principle generated by the quasi-classical gravity corrections to the QCD vacuum energy $[15,16]$. Very recently, it was understood that the unknown non-perturbative dynamics of the quantum-topological and quantum-wave modes of the YM vacuum could also be responsible for (partial or complete) compensation of the QCD instanton vacuum energy to the ground state energy of the Universe at macroscopic length scales and thus may be tightly related the colour confinement phenomenon $[16,17]$.

The major goal of the present paper is to study dynamical properties of the spatiallyinhomogeneous wave modes propagating in the spatially-homogeneous YM condensate incorporating interactions between these two components in the simplest pure YM SU(2) theory. Note, the YM wave modes are interpreted as particles after a quantisation procedure giving rise to the ultra-relativistic YM plasma, and our purpose is to study the plasma properties consistently taking into account its interactions with the YM condensate considered as a non-trivial background.

We consider the simplest situation when $\mathrm{SU}(2)$ is the complete gauge symmetry group. Note, the equations of motion of such a theory are form-invariant w.r.t. SU(2) gauge transformations whereas their solutions are not required to have exactly same symmetry as the equations. This situation is called spontaneous symmetry breaking (SSB). The appearance of the homogeneous and isotropic YM condensate is a specific case of the SSB. Namely, the condensate by itself is not gauge invariant, therefore, breaks the initial symmetry of the Lagrangian $\mathrm{SU}(2) \otimes \mathrm{SO}(3)$ down to the global $\mathrm{SO}(3)$ group of spatial 3-rotations.

The basic new result of our study is observation that the interactions between the small YM waves and large condensate in a simple SU(2) YM theory trigger a significant energy transfer in one particular direction, namely, from the condensate to the wave modes. Such a specific energy swap effect between the two YM subsystems may have serious consequences to the theory of non-perturbative YM vacuum and, in particular, may have important phenomenological implications e.g. in the theory of QCD phase transition in early Universe and in particle production mechanisms in the hot cosmological plasma. One 
may further extend this first simple analysis incorporating effects of mutual interactions between different wave modes. Certainly, the latter should be taken into account in a complete theory of ultra-relativistic gluon plasma.

To start with, we have constructed the exact quasi-classical equations for the wave modes and the condensate in the Hamilton gauge, and investigated them in the linear approximation (in small wave amplitudes limit). In this case, the equations of motion have a characteristic form of Mathieu equations having certain regions of parametric resonance instability which leads to an increase of amplitude of the waves. As was argued above, the constraints written for the system of interacting homogeneous YM condensate and inhomogeneous waves do not allow to exclude the longitudinal modes, such that these extra d.o.f.'s acquire their own dynamical properties due to interactions between the two subsystems.

As a consequence of energy conservation, an increase of the YM waves energy reflected in a corresponding increase of their amplitudes has to be accompanied by a corresponding decrease of the YM condensate energy. In particular, this fact must be taken into account in derivation of the next-to-linear condensate equation of motion where the "back reaction" effect of the wave modes to the condensate is consistently incorporated. The numerical analysis of the resulting system of equations has indeed revealed the energy swap effect satisfying the energy conservation: a decrease of the condensate energy is exactly compensated by an increase of energy attributed to the wave modes, which is an important test of our calculations.

In addition, equations of motion for pure gluon plasma (without the YM condensate) in the $\mathrm{SU}(3)$ gauge theory have been derived. It turned out that the third-order interaction terms between wave modes alone lead to excitation of a local dynamical (constituent) gluon mass whose expression has been obtained in a simple renormalized form and is shown to be consistent with the corresponding thermodynamic prediction. An inclusion of the secondorder interactions leads to dynamical mass terms in a time non-local form. Also, the exact integro-differential equations for the condensate and waves evolution incorporating both second- and third-order interaction terms in waves have been obtained in the $\mathrm{SU}(2)$ theory and can further be studied numerically which is a subject of future studies.

Note, a generalisation of these studies to the SU(3) theory appears to be conceptually straightforward but technically much more involved since it incorporates (i) three overlapping condensates which are thus expected to interact strongly with each other and (ii) a larger variety of the wave modes compared to the $\mathrm{SU}(2)$ case, and this analysis should be eventually performed. In this work, we have considered the gluon plasma dynamics in the $\mathrm{SU}(3)$ neglecting the contributions from the overlapping condensates. The next non-trivial example is the $\mathrm{SU}(4)$ theory which contains two non-overlapping gauge $\mathrm{SU}(2)$ groups and hence gives rise to two non-interacting condensates to the leading order in waves. Such a model has been discussed in some major details in this work. Note, that in the SU(4) toy-model we have disregarded all possible overlapping condensates in order to consider some basic features of heterogenic multi-condensate systems although a more realistic study involving overlapping condensates is necessary.

We have looked also into the question of gauge dependence of the YM condensate and waves. In fact, the YM condensate can be consistently extracted only in the Hamilton 
gauge where $A_{0}(x)=0$. Departing from this statement, we have proposed a new method which allows to extract the condensate and waves in arbitrary gauge and connect them to the condensate and waves evaluated in the Hamilton gauge. In short, for non-degenerate YM "condensate + waves" systems it is possible to establish a firm connection between the canonical results in the Hamilton gauge and those in arbitrary gauge by absorbing the gauge dependence (an infinitesimal depart from the Hamilton gauge is encoded by the gauge-fixing function $\left.\delta \theta\left(x^{\mu}\right)\right)$ into a coordinate transformation from $x^{\mu}$ to new abstract coordinates $z^{\mu}=z^{\mu}\left(x^{\mu}, \theta\left(x^{\mu}\right)\right)$. In new coordinates, the YM field $A_{\mu}(z)$ in arbitrary gauge is constrained in the same way as is typically done in the Hamilton gauge, i.e. $A_{0}(z)=0$, whereas $z^{\mu}$ absorbs the information about an underlined gauge choice. Then, the YM condensate and waves are consistently defined in such $z$-space and then transformed back to usual space-time. This approach has allowed to define the condensate in arbitrary gauge as a functional of the YM solution in the Hamilton gauge and the gauge-fixing function. This therefore justifies the rest of our studies of YM dynamics performed in the Hamilton gauge which is the most convenient in the present setting.

Further, we have generalised our study to the maximally $(\mathcal{N}=4)$ supersymmetric YM (SYM) theory (see e.g. ref. [25]). As one of the specific features of this theory is its conformality such that its $\beta$-function disappears (i.e. the coupling constant does not acquire radiative corrections and therefore does not run), which significantly simplifies our calculations. The $\mathcal{N}=4$ SYM theory includes four different fermion fields, three scalar and pseudo-scalar fields. We have shown, both numerically and analytically, that interactions of supersymmetric wave modes with the YM condensate lead to a similar energy swap effect from the YM condensate to the (pseudo)scalar wave modes as it was earlier observed for the vector wave modes in $\mathrm{SU}(2)$ gauge theory. These findings strongly suggest that the observed dynamics in energy balance of the interacting YM system ("wave + condensate") is a general phenomenon and specific property inherent to YM dynamics. Inclusion of coloured fermion modes into our quasi-classical analysis is relevant for particle production mechanisms in early Universe and will be done elsewhere.

Finally, we have looked into conformal time dynamics of the free YM condensate (without waves) on expanding background of the Friedmann Universe. A non-linear oscillatory behaviour of the condensate fluctuations contributing to the radiation-type matter has turned out to be unstable w.r.t. quantum radiative corrections. So we have incorporated the leading vacuum polarisation effects at one-loop and considered such a log-corrected quasi-free condensate dynamics. As a result, the condensate contribution to the energy density in this case becomes constant in time instead (and positive!) which is in accordance with the vacuum equation of state and can be used to eliminate large negative topological vacua contributions possibly providing a smallness of the observed cosmological constant. In order to investigate the latter possibility one should further incorporate the wave contributions together with the vacuum polarisation effects.

One should notice the following important point. A specific character of the considered SSB mechanism by means of the YM condensate compared to the traditional versions of the SSB (e.g. the quark-gluon condensate in spontaneous chiral symmetry breaking of the Higgs condensate in spontaneous electroweak symmetry breaking) is as follows. In other 
known versions one deals with the SSB of the vacuum which remains Lorentz-invariant, i.e. stationary in time and spatially-homogeneous and isotropic in 3-space with energymomentum tensor invariant w.r.t. Lorentz transformations (the well-known $\Lambda$-term). In our case, the YM condensate in its present form (i.e. without including vacuum polarisation effects) is not Lorentz-invariant due to a non-stationary character of the corresponding energy momentum tensor (it behaves as a radiation-type matter as the Universe expands).

As was mentioned above, it turned out that an inclusion of the vacuum polarisation effects dramatically change the time dependence of the YM condensate and its energy density - the latter becomes stationary in time and emerges as an extra constant (positive) contribution to the Lorentz-invariant $\Lambda$-term (i.e. satisfies to the vacuum equation of state $p=-\varepsilon$ ) although the condensate $U$ by itself is non-stationary which is therefore an instanton-type solution (This is due to the fact that the equations of motion for the free condensate are not form-invariant under small quantum fluctuations.) So, the Lorentz-invariance of the energy-momentum tensor of such a quasi-free condensate $U(t)$ is restored by the vacuum polarisation effects. For this very first study, the latter argument motivates our simplest choice of initially free Lorentz non-invariant YM condensate (with no vacuum polarisation incorporated) which is spatially-homogeneous and isotropic. At the next step, it would be important to incorporate vacuum polarisation and the wave dynamics on the same footing as it may give an access to topological effects, e.g. to a dynamical compensation of the topological and condensate excitations. Note, we are focused now on the simplest version of the SSB, but the YM condensate can, in principle, be spatially-inhomogeneous and at the same time not possess a wave structure (i.e. not be a superposition of waves). An analysis of non-homogeneous condensates could also be a good point for future studies.

Note also that if the ground state does not have a space-time symmetry of the initial theory (likewise, our free YM condensate is not Lorentz-invariant) then the conditions of the Goldstone theorem do not apply to this case of SSB. Therefore, there are no arguments to expect an appearance of the massless Goldstone modes after the SSB in this case. However, instead of the Goldstone effect we observe another interesting effect: the SSB in our case leads to a reconstruction of the excitations spectrum such that the underlined degrees of freedom of the initial YM theory (YM potentials) get reclassified into a new set of physical 3-tensor, 3-vector and 3-scalar d.o.f.'s. whose dynamics has been studied here.

The paper is organised as follows. In section 2, the generic properties of the YM dynamics in both degenerate (without the condensate) and non-degenerate interacting YM system "condensate + waves" in the Hamilton gauge have been discussed. Section 3 is devoted to a formulation of the interacting YM theory in arbitrary gauge and its important relation to the canonical framework developed in subsequent sections. Section 4 contains a thorough analytical and numerical analysis of the $\mathrm{SU}(2)$ "condensate + waves" system in the Hamilton gauge in the limit of small wave amplitudes compared to the condensate in zeroth, quasi-linear and next-to-linear approximations in wave modes. In section 5 we have discussed physics of the gluon plasma (without the condensate) and dynamical gluon mass generation due to wave self-interactions in different orders as well as the exact YM equations of motion of the "condensate + waves" system accounting for all the higher- 
order interaction terms. In section 6 we apply the quasi-classical approach to the $\mathcal{N}=4$ conformal Super-Yang-Mills theory with the condensate. Section 7 contains a thorough discussion of cosmological implications of the YM condensate with and without the vacuum polarisation effects as well as discusses typical condensate decay time scales due to condensate-wave interactions in quasi-linear approximation. Section 8 provides an overview of the simplest two-condensate model based upon SU(4) gauge theory. A few concluding remarks were made in section 9. Appendix A is devoted to details of the Hamilton formulation of a degenerate YM theory where the YM propagator has been derived by using the method of infinitesimal parameter. Finally, canonical quantisation of the YM wave modes in the classical YM condensate has been performed in appendix B as a consistency check of the quasi-classical framework developed in this work.

\section{Overview of Yang-Mills theory in the Hamilton gauge}

\subsection{Degenerate Yang-Mills system}

Let us remind a few important basics of the classical degenerate YM theory useful for the forthcoming analysis below. Consider first the simplest non-Abelian SU(2) gauge symmetry group. The gauge invariant Lagrangian of the corresponding YM field is

$$
\mathcal{L}=-\frac{1}{4} F_{\mu \nu}^{a} F_{a}^{\mu \nu}
$$

where

$$
F_{\mu \nu}^{a}=\partial_{\mu} A_{\nu}^{a}-\partial_{\nu} A_{\mu}^{a}+g e^{a b c} A_{\mu}^{b} A_{\nu}^{c}
$$

is the YM stress tensor with isotopic $a=1,2,3$ and Lorentz $\mu, \nu=0,1,2,3$ indices. So in the degenerate case there are twelve equations of motion

$$
\partial^{\mu} F_{\mu \nu}^{a}-g F_{\mu \nu}^{b} e_{a b c} A_{c}^{\mu}=0
$$

Consider first the simplest (ghost-free) Hamilton gauge. Note, the Hamilton gauge is the only known gauge which enables us to formulate the YM theory in the Heisenberg representation consistently beyond the perturbation theory (for more details, see e.g. ref. $[18,26])$. Indeed, the Heisenberg representation is the most useful and appropriate one in the analysis of real-time evolution of the homogeneous YM condensate. Imposing the Hamilton gauge condition

$$
A_{0}^{a}=0,
$$

one ends up with nine equations of motion

$$
\partial_{0} F_{0 k}^{a}=\partial_{i} F_{i k}^{a}-g F_{i k}^{b} e_{a b c} A_{i}^{c}
$$

and three additional constraint equations in the form of first integrals of motion

$$
\partial^{0}\left(\partial^{i} F_{i 0}^{a}-g F_{i 0}^{b} e_{a b c} A_{c}^{i}\right)=0,
$$


such that the total time derivative can be eliminated in the non-gauged (before the gauge is fixed) case, i.e.

$$
\partial^{i} F_{i 0}^{a}-g F_{i 0}^{b} e_{a b c} A_{c}^{i}=0 .
$$

It is worth to mention shortly a few important aspects of the YM quantum theory in the Hamilton gauge. Typically, such a theory is formulated in terms of the functional (or path) integral [18]. After introducing the Hamilton gauge into the path integral for the YM field, one defines the $S$-matrix and the YM propagator. As one of the attractive features of the Hamilton gauge, the asymptotic states of such $S$-matrix automatically contain transverse modes only. Then, the YM propagator is defined as a Green function of the equations of motion in the Hamilton gauge. The longitudinal mode gives a certain contribution to the YM propagator while it naturally disappears in the asymptotic states.

Below we adopt the following theoretically consistent and pragmatic approach which has certain methodological advantages in the analysis of interacting YM "waves + condensates" systems compared to the standard path integral formulation.

- Firstly, following to the Bohr's correspondence principle one starts with the pure YM Lagrangian in the Hamilton gauge and writes down the Lagrange equations of motion in the operator form;

- Secondly, since in the Hamilton gauge the zeroth component of the YM field $A_{0}$ is absent in the YM Lagrangian, there are no explicit constraint equations in the corresponding system of Lagrange equations. Such equations of constraint can be instead obtained as integrals of motion of the Lagrange equations;

- Thirdly, starting from the Lagrange formulation of the YM theory one easily turns to the Hamilton (or canonical) formulation and the canonical quantisation procedure.

Let us discuss the third point of the above scheme in more detail. In the case of free YM field (without its interactions with the condensate), the free (non-interacting) longitudinal YM mode does not have any proper frequency and dispersion, thus this mode is aperiodic in Minkowski space without the presence of YM condensate. Such a property has the following three important consequences: (1) the free longitudinal mode does not contribute to the Hamiltonian; (2) it is impossible to calculate its contribution to the YM propagator as a vacuum expectation value of the time-ordered YM field operator product; (3) there is a related issue with canonical quantisation. The first consequence is one of the advantages of the Hamilton gauge which automatically excludes any non-physical degrees of freedom from the YM Hamiltonian. The other two consequences point out to the fact that the YM theory in the Hamilton gauge cannot be constructed according to standard algorithms of a non-degenerate field theory.

One could advise a simple alternative and methodologically elegant way to resolve the latter issue: for a YM system without the presence of a homogeneous YM condensate one can introduce extra "virtual" infinitesimal terms (proportional to a single infinitesimal parameter $\xi$ ) into the YM Lagrangian in the Hamilton gauge. These terms can be chosen in such a way that the longitudinal mode acquires a small dispersion proportional to the 
small parameter $\xi$. Further, this enables to perform the canonical quantisation procedure in a standard way and to calculate a contribution of this mode to the YM propagator as a vacuum expectation value of the time-ordered YM field operator product. In the end, the infinitesimal parameter can be safely turned to zero $\xi \rightarrow 0$ leading to exactly the same $S$ matrix as the one defined in the framework of standard path integral formulation. Such an approach therefore leads to theoretically consistent results and enables one to work in the scheme described above. The method of infinitesimal parameter is described in appendix A in more detail.

\subsection{Non-degenerate Yang-Mills system: "condensate + waves"}

Let us now consider an interacting non-degenerate YM system "condensate + wave" in the pure $\mathrm{SU}(2)$ gauge theory. Here, the situation is different from the degenerate case described above in section 2.1. Namely, as will be explicitly shown below in section 4.2.2 there is a new effect of dynamical generation of the longitudinal plasma waves as collective excitations of macroscopic medium (e.g. condensate) also known as plasmons. The latter effect is well-known in physics of ordinary plasma $[20,21]$ and the quark-gluon plasma $[22,23]$ (see also ref. [24] and references therein). The longitudinal YM waves acquire both proper frequency (proportional to density of the medium) and dispersion (proportional to thermal wave velocity squared) and thus should be properly taken into consideration.

Now consider how the homogeneous YM condensate can be extracted from the YM field in the Hamilton gauge. It has been demonstrated in refs. [8-10] that due to isomorphism of the isotopic $\mathrm{SU}(2)$ group and the $\mathrm{SO}(3)$ group of spatial 3-rotations, the unique (up to rescaling) SU(2) YM configuration can be parameterized in terms of a scalar time-dependent spatially-homogeneous field. Indeed, such an isomorphism enables one to introduce a mixed space-isotopic orthonormal basis $e_{i}^{a}$ in which the YM vector field $A_{\mu}^{a}$ transforms into a tensor field $A_{i k}$ with two spatial indices ${ }^{1} i$ and $k$ as follows

$$
e_{i}^{a} A_{k}^{a}=A_{i k}, \quad e_{i}^{a} e_{k}^{a}=\delta_{i k}, \quad e_{i}^{a} e_{i}^{b}=\delta_{a b} .
$$

Then, the resulting spacial tensor $A_{i k}$ can be separated into two parts

$$
A_{i k}(t, \vec{x})=\delta_{i k} U(t)+\widetilde{A}_{i k}(t, \vec{x}),
$$

Here, the first spatially-homogeneous time-dependent scalar field $U(t)$ corresponds to the YM condensate and can be found as an average over a substantially large 3-space domain $\Omega \rightarrow \infty$

$$
U(t) \equiv \frac{1}{3} \delta_{i k}\left\langle A_{i k}(t, \vec{x})\right\rangle_{\vec{x}}, \quad\left\langle A_{i k}(t, \vec{x})\right\rangle_{\vec{x}}=\frac{\int_{\Omega} d^{3} x A_{i k}(t, \vec{x})}{\int_{\Omega} d^{3} x},
$$

In what follows, we consistently relate such an average procedure over 3 -spacial domain $\langle\ldots\rangle_{\vec{x}}$ with the average over the state vector $\langle\ldots\rangle$

$$
\langle\ldots\rangle \equiv\langle\Psi|\ldots| \Psi\rangle \sim\langle\ldots\rangle_{\vec{x}} .
$$

\footnotetext{
${ }^{1}$ For the spacial components, one does not distinguish upper and lower indices while repeated indices are summed up by default.
} 
It is important to notice that the condensate effectively removes the degeneracy of the underlined YM theory reducing the initial $\mathrm{SU}(2) \otimes \mathrm{SO}(3)$ symmetry of the YM Lagrangian to the global $\mathrm{SO}(3)$ symmetry of spacial rotations. The second quantum-wave part

$$
\widetilde{A}_{i k}=\widetilde{A}_{i k}(t, \vec{x}) \equiv A_{i k}(t, \vec{x})-\left\langle A_{i k}(t, \vec{x})\right\rangle_{\vec{x}}
$$

is spatially-inhomogeneous and describes motion of YM quanta, namely, physical particles after quantisation. It turns out that due to interactions between the YM condensate and waves, the longitudinal d.o.f.'s which is unphysical in the degenerate theory are parametrically excited and must be treated as physical ones.

Connections between the YM condensate and classical electric $E$ and magnetic $H$ fields can be easily derived, by definition of the YM potentials, e.g.

$$
\partial_{0} U=-\frac{1}{3}\left\langle E_{k k}\right\rangle, \quad U^{2}=\frac{1}{3 g}\left\langle H_{k k}\right\rangle,
$$

such that the condensate emerges as a mixture of $E$ and $H$ components. Analogous connections between the wave modes and the fields $E$ and $H$ can be obtained in a similar way.

Note, the above procedure remains valid also for the $\mathrm{SU}(3)$ gauge theory which however is much more complicated and contains three different but overlapping $\mathrm{SU}(2)$ subgroups isomorphic to $\mathrm{SO}(3)$. This situation potentially gives rise to three strongly interacting condensates, and needs to be analyzed separately. In a generic $\mathrm{SU}(N)$ model with $N>3$ the number of non-overlapping and overlapping $\mathrm{SU}(2)$ subgroups can be even larger which leads to formation of the heterogenic system of a few YM condensates. The simplest case of such a heterogenic system with two non-overlapping condensates is the $\mathrm{SU}(4)$ gauge theory. The latter has been discussed below in section 8 without an account for overlapping condensates.

\section{Yang-Mills theory in an arbitrary gauge}

In the previous section, we have discussed generic properties of the YM condensate theory in the Hamilton gauge and in isotopic $\mathrm{SU}(2)$ symmetry. Now we turn to an algorithm which allow us to describe YMC dynamics and YM waves in arbitrary gauge in connection to that known in the Hamilton gauge. Here, we consider the quasi-classical limit of the YM theory so at this point we do not discuss issues with ghost fields which appear via radiative corrections in a proper quantum formulation.

\subsection{Degenerate Yang-Mills system}

Consider for simplicity a non-Abelian gauge group $\mathrm{SU}(N), N=2,3$ with a set of generators $T_{a}$, which contains one isotopic $\mathrm{SU}(2)$ subgroup. In a degenerate theory, the corresponding YM field operator $A_{\mu} \equiv T^{a} A_{\mu}^{a},\left\langle A_{\mu}\right\rangle_{\vec{x}}=0$ with four Lorentz components $\mu=0,1,2,3$ contains only two physical components which can be identified transverse polarizations of the respective quanta. Recall, the local gauge transformation condition and the covariant 
derivative of the degenerate theory are given by

$$
\begin{aligned}
A_{\mu}^{(\theta)} & =G A_{\mu}^{\left(\theta_{0}\right)} G^{-1}+\frac{i}{g}\left(\partial_{\mu} G\right) G^{-1}, & D_{\mu} & =\hat{\partial}_{\mu}+i g A_{\mu}^{\left(\theta_{0}\right)}, \\
G \equiv G(x) & =\exp [i g \delta \theta(x)] \simeq \mathrm{I}+i g \delta \theta(x)+\ldots, & \delta \theta(x) & =T^{a} \delta \theta^{a}(x),
\end{aligned}
$$

The initial gauge $\mathrm{SU}(N)$ symmetric YM Lagrangian

$$
\mathcal{L}=-\frac{1}{2} \operatorname{Tr}\left[F_{\mu \nu} F^{\mu \nu}\right], \quad F_{\mu \nu} \equiv-\frac{1}{i g}\left[D_{\mu}, D_{\nu}\right]=\partial_{\mu} A_{\nu}-\partial_{\nu} A_{\mu}+g\left[A_{\mu}, A_{\nu}\right],
$$

is manifestly invariant under the above gauge transformation condition which takes the following infinitesimal form

$$
A_{\mu}^{(\theta)}=A_{\mu}^{\left(\theta_{0}\right)}+i g\left[\delta \theta, A_{\mu}^{\theta_{0}}\right]-\partial_{\mu} \delta \theta .
$$

Above, $A_{\mu}^{\left(\theta_{0}\right)}$ is the YM field in a given reference gauge. In what follows, it will be convenient to choose a simple reference gauge where the YM solutions of the non-degenerate theory are assumed to be known. So whenever it is reasonable we will use the simplest Hamilton gauge $\theta_{0} \equiv \theta_{H}$ as our reference gauge assuming that the corresponding solutions are known.

As was advocated above in the Hamilton gauge, due to isomorphism of the local isotopic $\mathrm{SU}(2)$ (sub)group and the global SO(3) group of spatial 3-rotations, the YM quanta condensation can effectively induce a homogeneous classical background field, the YM condensate, whose vacuum expectation value is non-trivial. As was discussed above, the condensate removes the degeneracy of the underlined YM theory and effectively breaks the symmetry of initial YM Lagrangian down to the $\mathrm{SO}(3)$ rotation symmetry. In this case, besides two physical transversely polarised modes one extra physical wave d.o.f. gets parametrically excited in the YM condensate and can be associated with the longitudinally polarised plasma mode. Therefore, we effectively end up with three independent YM wave operators $\widetilde{A}_{k}(t, \vec{x})$ where index $k$ was associated with three spacial components in the Hamilton gauge. In what follows, we consider a non-degenerate theory and rely on the fact that the number of physical modes $\widetilde{A}_{k}^{a}(t, \vec{x})$ is equal to $3\left(N^{2}-1\right)$ and gauge-independent which allows us to determine the YM condensate dynamics in an analogical way to that in the Hamilton gauge.

In order to construct a consistent theory of the interacting system "condensate + waves" in arbitrary gauge let us introduce an abstract Minkowski 4-space with coordinates depending on the gauge-fixing function $\delta \theta$, i.e.

$$
z_{\alpha}=z_{\alpha}(t, \vec{x} ; \delta \theta(t, \vec{x})) \equiv\{\tau, \vec{z}\}, \quad \alpha=0,1,2,3,
$$

which are related to usual space-time coordinates $x_{\mu}=\{t, \vec{x}\}$ as

$$
d z_{\alpha}=\varepsilon_{\alpha}^{\mu} d x_{\mu}, \quad \varepsilon_{\alpha}^{\mu}=\frac{\partial z_{\alpha}}{\partial x_{\mu}}, \quad \varepsilon_{\mu \alpha}^{-1}=\frac{\partial x_{\mu}}{\partial z^{\alpha}} .
$$

The new Lorentz-covariant coordinate transformation matrix $\varepsilon_{\alpha}^{\mu}$ is normalized as

$$
\varepsilon_{\alpha}^{\mu}=\varepsilon_{\alpha}^{\mu}(\tau(t, \vec{x} ; \delta \theta), \vec{z}(t, \vec{x} ; \delta \theta)), \quad \varepsilon_{\alpha}^{\mu} \varepsilon_{\mu \beta}^{-1}=g_{\alpha \beta}, \quad \varepsilon_{\mu}^{\alpha}\left(\varepsilon_{\nu \alpha}\right)^{-1}=g_{\mu \nu} .
$$


In what follows, we call such an abstract $4 \mathrm{D}$ space as the $z$-space. In general, the basis transformation matrix $\varepsilon_{\mu \alpha}$ depends on the gauge-fixing function $\delta \theta=\delta \theta(t, \vec{x})$ and can therefore effectively absorb all the information about the underlined gauge choice. In particular, in the Hamilton gauge corresponding to $\theta \rightarrow \theta_{H}$ it takes a simple form

$$
\varepsilon_{\mu \alpha}=\delta_{\mu \alpha},
$$

which means that $z$-space simply coincides with usual coordinate 4 -space in the Hamilton gauge

$$
\lim _{\delta \theta \rightarrow 0} z_{\alpha}(t, \vec{x} ; \delta \theta)=x_{\alpha}
$$

Let us now define the $z$-space projections of the YM field $A_{\mu}^{(\theta)}$ and the stress tensor $F_{\mu \nu}^{(\theta)}$ in a given gauge as follows

$$
A_{\alpha}^{(\theta)}(\tau, \vec{z}) \equiv\left(\varepsilon_{\alpha}^{\mu}\right)^{-1} A_{\mu}^{(\theta)}, F_{\alpha \beta}^{(\theta)}(\tau, \vec{z}) \equiv\left(\varepsilon_{\alpha}^{\mu}\right)^{-1}\left(\varepsilon_{\beta}^{\nu}\right)^{-1} F_{\mu \nu}^{(\theta)}=\delta_{\alpha} A_{\beta}^{(\theta)}-\delta_{\beta} A_{\alpha}^{(\theta)}+g\left[A_{\alpha}^{(\theta)}, A_{\beta}^{(\theta)}\right],
$$

where

$$
\delta_{\alpha} \equiv \frac{\partial}{\partial z^{\alpha}}=\varepsilon_{\mu \alpha}^{-1} \partial^{\mu},
$$

The equations of motion in $z$-space

$$
\delta^{\alpha} F_{\alpha \beta}^{(\theta)}(\tau, \vec{z})+i g\left[A^{\alpha(\theta)}(\tau, \vec{z}), F_{\alpha \beta}^{(\theta)}(\tau, \vec{z})\right]=0,
$$

are covariant w.r.t. local gauge transformations of the YM field from a chosen reference gauge $\theta^{\prime}$ to a given gauge $\theta$ in infinitesimal form

$$
A_{\alpha}^{(\theta)}(\tau, \vec{z})=A_{\alpha}^{\left(\theta^{\prime}\right)}(\tau, \vec{z})+i g\left[\delta \theta, A_{\alpha}^{\left(\theta^{\prime}\right)}(\tau, \vec{z})\right]-\delta_{\alpha} \delta \theta .
$$

The particular gauge null-transform to/from the Hamilton gauge $\theta^{\prime}=\theta_{H}$

$$
\delta \theta_{[0]}=C \exp \left[i g \int A_{k}(t, \vec{x}) d x_{k}\right] .
$$

corresponds to a closed loop in the space of gauge configurations such that the gauge field transforms to itself. In what follows, we assume that $\delta \theta \neq \delta \theta_{[0]}$ unless noted otherwise. For finite transformation, one writes consequently

$$
A_{\alpha}^{(\theta)}(\tau, \vec{z})=\frac{1}{i g} G\left(\hat{\delta}_{\alpha}+i g A_{\alpha}^{\left(\theta^{\prime}\right)}(\tau, \vec{z})\right) G^{-1} .
$$

At the next step, in a complete analogy with the Hamilton gauge the zeroth component of the gauge field in $z$-space is required to be zeroth in any gauge, i.e.

$$
A_{0}^{(\theta)}(\tau(t, \vec{x} ; \delta \theta), \vec{z}(t, \vec{x} ; \delta \theta))=0, \quad \delta \theta=\delta \theta(t, \vec{x}),
$$

such that the other three components $A_{k}^{(\theta)}(\tau, \vec{z}), k=1,2,3$ should be identified with physical YM operators. Starting from the Hamilton gauge as our reference gauge, the latter 
identification allows to consider a coordinate transformation of usual $4 \mathrm{D} x$-space to a new $4 \mathrm{D} z$-space as a gauge transformation, i.e.

$$
A_{k}^{(\theta)}(\tau(t, \vec{x} ; \delta \theta), \vec{z}(t, \vec{x} ; \delta \theta))=A_{k}^{\left(\theta_{H}\right)}(t, \vec{x}) \equiv A_{k}(t, \vec{x}), \quad \delta \theta=\delta \theta(t, \vec{x}),
$$

which provides a connection between the coordinate transformation matrix $\varepsilon_{\mu \alpha}$ and the gauge fixing function $\delta \theta$. Indeed, using this relation in eq. (3.10) one obtains the following constraint equations

$$
\left(\varepsilon_{\alpha}^{\mu}\right)^{-1} \partial_{\mu} \delta \theta(t, \vec{x})=-i g\left[\delta \theta(t, \vec{x}),\left(\varepsilon_{\alpha}^{k}\right)^{-1} A_{k}(t, \vec{x})\right]-\left(\delta_{\alpha}^{k}+\left(\varepsilon_{\alpha}^{k}\right)^{-1}\right) A_{k}(t, \vec{x}),
$$

in terms of the YM solution in the Hamilton gauge $A_{k}(t, \vec{x})$ in the Hamilton gauge. The relations (3.13) then determine the coordinate transformation matrix $\varepsilon_{\mu \alpha}$ and hence the complete YM solution in arbitrary gauge $A_{\mu}^{(\theta)}$ as functionals of the gauge fixing function $\delta \theta(t, \vec{x})$ and the YM solution in the Hamilton gauge $A_{k}(t, \vec{x})$, namely

$$
\bar{\varepsilon}_{\mu \alpha}(t, \vec{x})=\varepsilon_{\mu \alpha}\left[A_{k}(t, \vec{x}) ; \delta \theta(t, \vec{x})\right], \quad A_{\mu}^{(\theta)}(t, \vec{x})=-A_{k}(t, \vec{x}) \bar{\varepsilon}_{\mu k}(t, \vec{x}) .
$$

Therefore, the YM solution in arbitrary gauge is expressed in terms of the canonical solution in the Hamilton gauge and the transformation matrix $\bar{\varepsilon}_{\mu \alpha}(t, \vec{x})$ which absorbs all the information about the underlined gauge transformation.

\subsection{Non-degenerate Yang-Mills system: "condensate + waves"}

Based upon a direct analogy of the YM field consideration in ordinary space-time and in the Hamilton gauge made in the previous section, isomorphism of the global $\mathrm{SO}(3)$ symmetry group of 3-rotations in $z$-space and local $\mathrm{SU}(2)$ subgroup of $\mathrm{SU}(N), N=2,3$ gauge group enables us to define the YM condensate and YM waves in arbitrary gauge. Indeed, by employing the mixed space-isotopic orthonormal basis $e_{i}^{a}$ defined in eq. (2.7) one writes for the physical YM operators in $z$-space

$$
A_{k}^{(\theta)}(\tau, \vec{z}) \equiv T^{a} A_{k}^{a(\theta)}(\tau, \vec{z})=\mathcal{T}_{i} A_{i k}^{(\theta)}(\tau, \vec{z}),
$$

where

$$
\mathcal{T}_{i} \equiv T^{a} e_{i}^{a}, \quad A_{i k}^{(\theta)}(\tau, \vec{z})=A_{i k}(t, \vec{x})=\delta_{i k} W^{(\theta)}(t)+\widetilde{A}_{i k}^{(\theta)}(t, \vec{x})
$$

in terms of the homogeneous YM condensate $W^{(\theta)}(t)$ and non-homogeneous YM waves $\widetilde{A}_{i k}^{(\theta)}(t, \vec{x})$ which coincide with $U(t)$ and $\widetilde{A}_{i k}(t, \vec{x})$ in the Hamilton gauge limit, respectively, corresponding to $\delta \theta \rightarrow \delta \theta_{[0]}$. The time-dependent scalar field $W^{(\theta)}(t)$ is defined normal way as an average over a large $z$-spacial domain $\Theta \rightarrow \infty$ at whose boundary all the fields (more precisely, wave excitations) are vanishingly small

$$
W^{(\theta)}(t) \equiv \frac{1}{3} \delta_{i k}\left\langle A_{i k}^{(\theta)}(\tau, \vec{z})\right\rangle_{\vec{z}}, \quad\left\langle A_{i k}^{(\theta)}(\tau, \vec{z})\right\rangle_{\vec{z}}=\frac{\int_{\Theta} d^{3} z A_{i k}^{(\theta)}(\tau, \vec{z})}{\int_{\Theta} d^{3} z}=\frac{\int_{\Omega} d^{3} x|\bar{\varepsilon}| A_{i k}(t, \vec{x})}{\int_{\Omega} d^{3} x|\bar{\varepsilon}|}
$$


where $A_{i k}(t, \vec{x})$ is the known YM solution in the Hamilton gauge

$$
A_{i k}(t, \vec{x})=\delta_{i k} U(t)+\widetilde{A}_{i k}(t, \vec{x}),
$$

and the Jacobian of the 3-volume element transformation is

$$
|\bar{\varepsilon}| \equiv\left|\operatorname{det}\left\{\bar{\varepsilon}_{m}^{l}(t, \vec{x})\right\}\right|=\left|\frac{\partial\left(z_{1}, z_{2}, z_{3}\right)}{\partial\left(x_{1}, x_{2}, x_{3}\right)}\right| .
$$

The YM condensate in arbitrary gauge finally reads

$$
W^{(\theta)}(t)=\frac{1}{3} \delta_{i k}\left\langle A_{i k}(t, \vec{x})\right\rangle_{\vec{z}}=U(t)+\frac{1}{3} \delta_{i k}\left\langle\widetilde{A}_{i k}(t, \vec{x})\right\rangle_{\vec{z}} \neq U(t), \quad \delta \theta \neq \delta \theta_{[0]},
$$

where the last term vanishes in the Hamilton gauge $\delta \theta \rightarrow \delta \theta_{[0]}$ corresponding to $|\bar{\varepsilon}| \rightarrow 1$. The physical quantum-wave components are then given by

$$
\widetilde{A}_{i k}^{(\theta)}(t, \vec{x}) \equiv A_{i k}(t, \vec{x})-\delta_{i k} W^{(\theta)}(t) \neq \widetilde{A}_{i k}(t, \vec{x}), \quad \delta \theta \neq \delta \theta_{[0]} .
$$

Therefore, in this formulation the quasi-classical YM theory in an arbitrary gauge can be constructed in analogous way to that in the Hamilton gauge. The latter analogy allows to extract the YM condensate and to study its properties from the universal perspectives. The suggested formulation establishes a tight connection between the YM condensate and waves in the Hamilton gauge and those in an arbitrary gauge. So in the coming sections we will be focused primarily on analysis of the YM dynamics in the Hamilton gauge and its extension to an arbitrary gauge is now conceptually straightforward.

\subsection{Non-local gauge transformations}

As a result of the above discussion, we observe that despite the initial field $A_{i k}^{(\theta)}(t, \vec{z})$ in the $z$-space coincides with the one in the Hamilton gauge in $A_{i k}(t, \vec{x})$ in the $x$-space by construction, the corresponding separate $\mathrm{YM}$ condensates and wave contributions do not coincide. Indeed, an extra weighting factor $|\bar{\varepsilon}(t, \vec{x})|$ appears in the average (3.20) such that it may lead to the appearance of additional contributions to the condensate $U(t)$ from nonvanishing averages of higher powers of the waves $\widetilde{A}_{i k}(t, \vec{x})$. This is a direct consequence of the fact that the gauge symmetry is dynamically broken by the condensation of YM quanta. Since the total energy of the interacting system "condensate + waves" is conserved, this means that the gauge fixing operator $\delta \theta$ acquires a dynamical role and describes possible energy exchanges between the YM condensate and waves, and in this case may play a role of the evolution operator.

Interestingly enough, this effect, has a close analogy to the real time evolution of the YM system in the fixed Hamilton gauge where energy of the YM condensate is dynamically transferred into the waves in the quasi-classical limit of small wave amplitudes as will be shown below. Let us pay a closer attention to a possible connection between the "gauge evolution" in abstract $z$-space and real time evolution of the non-degenerate "condensate + waves" system in usual space-time.

Consider a YM field in $z$-space fixed by constraints (3.13) in a given gauge $\theta \neq \theta_{H}$, i.e.

$$
A_{0}^{(\theta)}(z[\delta \theta(x)]) \equiv 0, \quad A_{k}^{(\theta)}(z[\delta \theta(x)])=A_{k}(x), \quad \delta \theta \neq \delta \theta_{[0]},
$$


where $z=\{\tau, \vec{z}\}$ and $x=\{t, \vec{x}\}$. Then, define another gauge $\theta^{\prime} \neq \theta, \theta^{\prime} \neq \theta_{H}$ such that the infinitesimal gauge transformation $\theta \rightarrow \theta^{\prime}$ takes the following generic non-local form

$$
A_{k}^{(\theta)}(z[\delta \theta])=A_{k}^{\left(\theta^{\prime}\right)}\left(z\left[\delta \theta^{\prime}\right]\right)+i g\left[\gamma\left[\delta \theta, \delta \theta^{\prime}\right], A_{k}^{\left(\theta^{\prime}\right)}\left(z\left[\delta \theta^{\prime}\right]\right)\right]-\delta_{k} \gamma\left[\delta \theta, \delta \theta^{\prime}\right],
$$

where $\delta \theta$ and $\delta \theta^{\prime}$ denote the gauge transforms corresponding to $\theta_{H} \rightarrow \theta$ and $\theta_{H} \rightarrow \theta^{\prime}$, respectively, and the non-local operator $\gamma\left[\delta \theta, \delta \theta^{\prime}\right]$ describes the gauge transform $\theta \rightarrow \theta^{\prime}$ with boundary condition

$$
\gamma[\delta \theta, \delta \theta] \equiv \delta \theta=C \exp \left[i g \int A_{k}^{(\theta)}(z[\delta \theta]) d z_{k}\right] .
$$

Following to the scheme above, one notices that the gauge transformations $\delta \theta$ and $\delta \theta^{\prime}$ are equivalent to coordinate transforms

$$
x \rightarrow z \equiv z[\delta \theta], \quad x \rightarrow z^{\prime} \equiv z\left[\delta \theta^{\prime}\right],
$$

respectively. Absorbing the gauge dependence into the $z$-coordinate transformation as usual

$$
A_{k}^{(\theta)}(z[\delta \theta(x)])=A_{k}^{\left(\theta^{\prime}\right)}\left(z\left[\delta \theta^{\prime}(x)\right]\right)=A_{k}(x),
$$

one observes that

$$
\varepsilon_{\mu k}^{-1}\left[\delta \theta^{\prime}(x)\right] \partial^{\mu} \gamma\left[\delta \theta(x), \delta \theta^{\prime}(x)\right]=i g\left[\gamma\left[\delta \theta(x), \delta \theta^{\prime}(x)\right], A_{k}(x)\right], \quad \varepsilon_{\mu k}^{-1}\left[\delta \theta^{\prime}(x)\right]=\frac{\partial x_{\mu}}{\partial z_{k}^{\prime}},
$$

which is valid for any $\delta \theta$ and $\delta \theta^{\prime}$. At last, for a given pair of non-intersecting nontrivial gauges

$$
\delta \theta^{\prime}(x) \neq \delta \theta(x)
$$

one could always find two different space-time points $x$ and $x^{\prime}$ for which

$$
\delta \theta^{\prime}(x)=\delta \theta\left(x^{\prime}\right)
$$

is satisfied. In this case, the functional relation (3.25) finally transforms to

$$
\varepsilon_{\mu k}^{-1}\left[A_{k}\left(x^{\prime}\right) ; \delta \theta\left(x^{\prime}\right)\right] \partial^{\mu} \gamma\left[\delta \theta(x), \delta \theta\left(x^{\prime}\right)\right]=i g\left[\gamma\left[\delta \theta(x), \delta \theta\left(x^{\prime}\right)\right], A_{k}(x)\right],
$$

where $\varepsilon_{\mu k}^{-1}$ has been found earlier in eq. (3.14). We therefore notice that the gauge evolution between two points $\theta$ and $\theta^{\prime}$ in space of non-equivalent gauge configurations driven by the non-local operator $\gamma\left[\delta \theta, \delta \theta^{\prime}\right]$ can be unambiguously mapped onto real physical motion between two space-time points $x$ and $x^{\prime}$ related via (3.26) in a fixed gauge $\theta$ whose evolution operator has the same form $\gamma\left[\delta \theta(x), \delta \theta\left(x^{\prime}\right)\right]$. Indeed, in the considered non-degenerate theory the values of the YM field in the Hamilton gauge defined in two different space-time points $A_{k}(x)$ and $A_{k}\left(x^{\prime}\right)$ have turned out to be related via the operator $\gamma$ which therefore acquires a dynamical role. A detailed development of this concept goes significantly beyond the scope of the present paper and will be done elsewhere. Instead, we turn to analysis of the YM condensate and waves in the Hamilton gauge. 


\section{Yang-Mills dynamics in the Hamilton gauge: one condensate model}

Below in this section as the very first step we consider the simplest one-condensate model in $\mathrm{SU}(2)$ gauge theory analysing consequently its dynamics in different limiting cases and approximations.

\subsection{Free condensate case}

To the zeroth order in small waves $\left|\widetilde{A}_{i k}\right| \ll|U|$, the representation (2.8) enables us to rewrite the Hamiltonian and YM equation of motion (2.4) for the real-time evolution of the YM condensate alone, $U=U(t)$. Namely,

$$
\mathcal{H}_{\mathrm{YM}} \simeq \mathcal{H}_{\mathrm{YMC}}=\frac{3}{2}\left[\left(\partial_{0} U\right)^{2}+g^{2} U^{4}\right], \quad \partial_{0} \partial_{0} U+2 g^{2} U^{3}=0 .
$$

Its numerical solution is shown in figure 1(left). The exact solution of the equation of motion for the "free" YM condensate is given by

$$
t=-\int_{U_{0}}^{U} \frac{d U}{\sqrt{g^{2} U_{0}^{4}-g^{2} U^{4}}}, \quad U(0)=U_{0}, \quad U^{\prime}(0)=0 .
$$

According to this result the free YM condensate can exist only in a free non-stationary slowly-oscillating state. Indeed, to a good accuracy, the latter exhibits a non-linear oscillation pattern and can be approximated by a quasi-harmonic function with frequency of oscillations depending on their amplitude, e.g.

$$
U \simeq U_{0} \cos \left(\omega t+\phi_{0}\right), \quad \omega \equiv \frac{2 \pi}{T_{U}}=k g U_{0}, \quad k=\frac{2 \pi}{B\left(\frac{1}{4}, \frac{1}{2}\right)} \simeq 1.2
$$

where $T_{U}$ is the period of YM condensate oscillations, and $B(x, y)$ is the Euler beta function. The maximal error of this approximation is limited by $\Delta U / U \lesssim 0.07$.

The energy spectrum of quasi-harmonic YM condensate fluctuations can be found in standard way from the Schrödinger steady-state equation and is shown in figure 1(right). Starting from the Hamiltonian density for the free (non-interacting) YM condensate (4.1) one arrives at the Schrödinger equation

$$
\left[\frac{1}{6} \frac{d^{2}}{d U^{2}}+\left(E-\frac{3}{2} g^{2} U^{4}\right)\right] \Psi=0 .
$$

It straightforward to show that the free YM condensate spectrum corresponds to a potential well of the fourth power. Numerical calculation provides us with the first few energy levels in the spectrum (see also, ref. [31]), e.g.

$$
E_{n}=\widetilde{E}_{n} \frac{g^{2 / 3}}{3^{1 / 3}}, \quad \widetilde{E}_{0} \simeq 0.5, \quad \widetilde{E}_{1} \simeq 1.9, \quad \widetilde{E}_{2} \simeq 3.7, \quad \widetilde{E}_{3}=5.8, \quad \widetilde{E}_{4}=8.13, \quad \ldots
$$

For practical use, it is convenient to come up with an approximate analytic formula for the lower end of this spectrum, e.g. in the following form

$$
\widetilde{E}_{n}=\left(1.06+0.395 n^{0.53}\right)(n+0.5), \quad n=0,1,2, \ldots
$$

The maximal error of this formula for the first thirty energy levels does not exceed $4 \%$. 

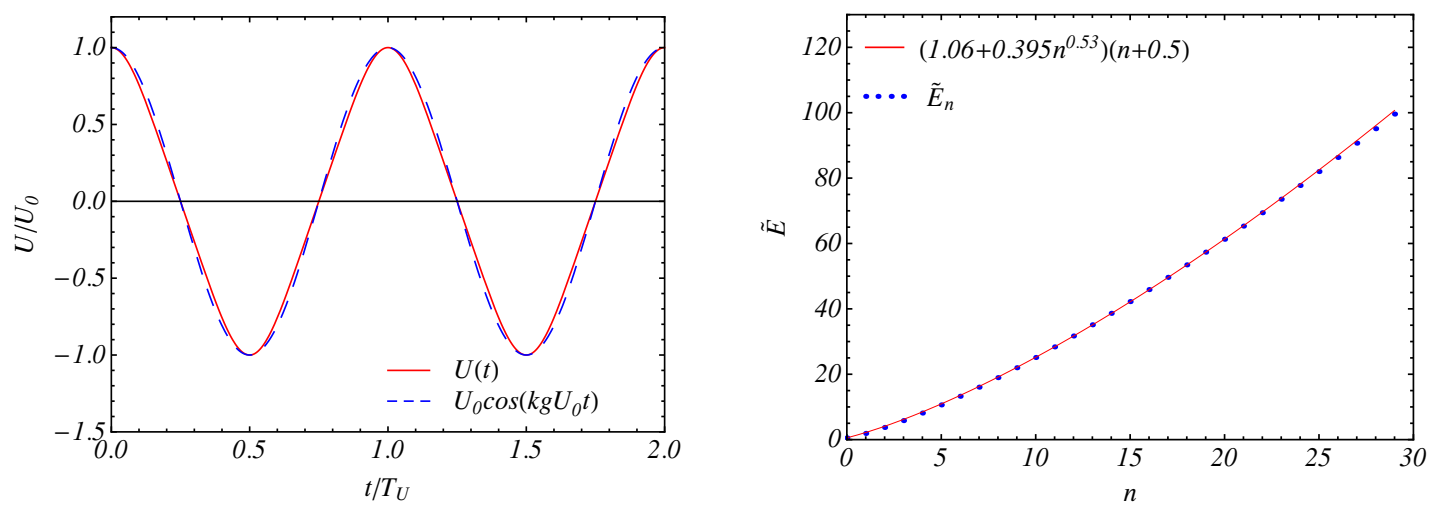

Figure 1. Numerical solution of the YM equation of motion (4.1) for the time dependence of free YM condensate potential, $U=U(t)$, and its analytical approximation (4.3) for a fixed initial phase $\phi_{0}=0$ (left) and numerical result for the energy spectrum of free YM condensate, $E_{n}$, and its continuous analytical approximation (4.6) (right). This plot is given in terms of dimensionless quantities and thus does not depend on initial value of the condensate $U_{0}$.

\subsection{Condensate and wave dynamics in the linear approximation}

Consider now the physically interesting and realistic case of the YM wave modes interacting with the YM condensate. We start with the linear (in waves) approximation where only interactions between YM wave modes and YM condensate are taken into account while interactions between different wave modes are not included. In practice, this situation corresponds to a YM system in the beginning of its real time evolution with very few wave modes such that the interactions between waves are negligibly small compared to "wavecondensate" interactions. Let us consider dynamics of such a linearized system in detail.

\subsubsection{First-order Yang-Mills equations of motion}

The all-order YM equation of motion (2.4) for the YM condensate, $U=U(t)$, interacting with the wave modes, $\widetilde{A}_{i k}$, can be written as follows

$$
\begin{aligned}
& -\delta_{l k}\left(\partial_{0} \partial_{0} U+2 g^{2} U^{3}\right)+\left(-\partial_{0} \partial_{0} \widetilde{A}_{l k}+\partial_{i} \partial_{i} \widetilde{A}_{l k}\right. \\
& -\partial_{i} \partial_{k} \widetilde{A}_{l i}-g e_{l m k} \partial_{i} \widetilde{A}_{m i} U-2 g e_{l i p} \partial_{i} \widetilde{A}_{p k} U \\
& \left.-g e_{l m i} \partial_{k} \widetilde{A}_{m i} U+g^{2} \widetilde{A}_{k l} U^{2}-g^{2} \widetilde{A}_{l k} U^{2}-2 g^{2} \delta_{l k} \widetilde{A}_{i i} U^{2}\right)+\left(-g e_{l m p} \partial_{i} \widetilde{A}_{m i} \widetilde{A}_{p k}\right. \\
& -2 g e_{l m p} \widetilde{A}_{m i} \partial_{i} \widetilde{A}_{p k}-g e_{l m p} \partial_{k} \widetilde{A}_{m i} \widetilde{A}_{p i}+g^{2} \widetilde{A}_{l i} \widetilde{A}_{i k} U+g^{2} \widetilde{A}_{l i} \widetilde{A}_{k i} U+g^{2} \widetilde{A}_{i k} \widetilde{A}_{i l} U \\
& \left.\quad-2 g^{2} \widetilde{A}_{i i} \widetilde{A}_{l k} U-g^{2} \delta_{l k} \widetilde{A}_{p i} \widetilde{A}_{p i} U\right)+g^{2}\left(\widetilde{A}_{l i} \widetilde{A}_{p k} \widetilde{A}_{p i}-\widetilde{A}_{p i} \widetilde{A}_{p i} \widetilde{A}_{l k}\right)=0 .
\end{aligned}
$$

The constraint equation (2.6) linearized in waves reads

$$
\partial_{i} \partial_{0} \widetilde{A}_{l i}-g e_{l m i} \partial_{0} U \widetilde{A}_{m i}+g e_{l m i} \partial_{0} \widetilde{A}_{m i} U+g e_{l m p} \partial_{0} \widetilde{A}_{m i} \widetilde{A}_{p i}=0 .
$$

Note, the equation (4.7) is separable by averaging over the Heisenberg state vector. Also, as the matter of perturbation theory approach the zeroth-order YM condensate equation (4.1) has to be fulfilled in order to find the equations of motion for the YM wave modes to the first (linear) approximation. 
It is convenient to turn to Fourier transforms for $\widetilde{A}_{i k}$ modes and expand them over the tensor basis [27]. In terms of symmetric and antisymmetric parts, the tensor field $\widetilde{A}_{i k}$ reads

$$
\widetilde{A}_{i k}=\psi_{i k}+e_{i k l} \chi_{l} .
$$

Then, we expand the Fourier transforms of antisymmetric $\chi_{l}$ and symmetric $\psi_{i k}$ modes into the tensor basis as

$$
\chi_{l}^{\vec{p}}=s_{l}^{\sigma} \eta_{\sigma}^{\vec{p}}+n_{l} \lambda^{\vec{p}}
$$

and

$$
\psi_{i k}^{\vec{p}}=\psi_{\lambda}^{\vec{p}} Q_{i k}^{\lambda}+\varphi_{\sigma}^{\vec{p}}\left(n_{i} s_{k}^{\sigma}+n_{k} s_{i}^{\sigma}\right)+\left(\delta_{i k}-n_{i} n_{k}\right) \Phi^{\vec{p}}+n_{i} n_{k} \Lambda^{\vec{p}},
$$

respectively, where the coefficients satisfy the following conditions

$$
Q_{i k}^{\lambda}=Q_{k i}^{\lambda}, \quad Q_{i i}^{\lambda}=0, \quad p_{i} Q_{i k}^{\lambda}=0, \quad p_{k} s_{k}=0 .
$$

In eqs. (4.10) and (4.11), the 3-vectors $n_{i}$ and $s_{k}$ are the longitudinal and transverse unit vectors, respectively, and $\vec{p}$ is the corresponding Fourier 3 -momentum. In what follows, we omit the Fourier momentum index $\vec{p}$.

Next, let us rewrite the general equation of motion (4.7) (after a proper subtraction of the YM condensate equation (4.1)) through the new d.o.f.'s to the linear (in waves) approximation as follows

$$
\partial_{0} \partial_{0} \psi_{\lambda}+p^{2} \psi_{\lambda}+i g p U Q^{\lambda \gamma} \psi_{\gamma}=0
$$

and

$$
\begin{aligned}
& \partial_{0} \partial_{0} \Lambda+2 i g p \lambda U+2 g^{2} U^{2}(2 \Phi+\Lambda)=0, \\
& \partial_{0} \partial_{0} \Phi+p^{2} \Phi+2 i g p \lambda U+2 g^{2} U^{2}(2 \Phi+\Lambda)=0, \\
& \partial_{0} \partial_{0} \lambda+p^{2} \lambda-i g p(2 \Phi+\Lambda) U+2 g^{2} U^{2} \lambda=0, \\
& \partial_{0} \partial_{0} \phi_{\sigma}+\frac{p^{2}}{2} \phi_{\sigma}-\frac{p^{2}}{2} e^{\sigma \gamma} \eta_{\gamma}-i g p U e^{\gamma \sigma} \phi_{\gamma}=0, \\
& \partial_{0} \partial_{0} \eta_{\sigma}+\frac{p^{2}}{2} \eta_{\sigma}-\frac{p^{2}}{2} e^{\gamma \sigma} \phi_{\gamma}+i g p U e^{\gamma \sigma} \eta_{\gamma}+2 g^{2} U^{2} \eta_{\sigma}=0,
\end{aligned}
$$

where the following shorthand notations

$$
e^{\alpha \beta} \equiv e_{i k m} s_{i}^{\alpha} n_{k} s_{m}^{\beta}, \quad Q^{\lambda \gamma} \equiv e_{l i p} n_{i} Q_{l k}^{\lambda} Q_{k p}^{\gamma},
$$

are adopted. Therefore, one arrives at the system of nine equations of motion for nine d.o.f.'s. Finally, the equations of constraint (4.8) can be conveniently transformed to the following explicit form in terms of new d.o.f.'s

$$
\begin{aligned}
\partial_{0}\left(i p \partial_{0} \Lambda-2 g \lambda \partial_{0} U+2 g \partial_{0} \lambda U\right) & =0 \\
\partial_{0}\left(i p \partial_{0} \phi_{\gamma}+i p e^{\gamma \sigma} \partial_{0} \eta_{\sigma}-2 g \eta_{\gamma} \partial_{0} U+2 g \partial_{0} \eta_{\gamma} U\right) & =0 .
\end{aligned}
$$


It is straightforward to check that these two constraints are automatically satisfied for a solution of the system of YM equations (4.13) and (4.14) (see appendix B for consistency checks of the corresponding equations of motion). Note, in a non-gauged YM theory, these constraints do not explicitly contain time derivatives, i.e.

$$
\begin{aligned}
i p \partial_{0} \Lambda-2 g \lambda \partial_{0} U+2 g \partial_{0} \lambda U & =0, \\
i p \partial_{0} \phi_{\gamma}+i p e^{\gamma \sigma} \partial_{0} \eta_{\sigma}-2 g \eta_{\gamma} \partial_{0} U+2 g \partial_{0} \eta_{\gamma} U & =0,
\end{aligned}
$$

which are thus the first integrals of motion.

For further considerations, it is instructive to represent quadratic Lagrangian and Hamiltonian densities of the SU(2) YM wave modes interacting with the YM condensate $U=U(t)$ in terms of the new d.o.f. as follows

$$
\begin{aligned}
& \mathcal{L}_{\mathrm{YM}}^{\text {waves }}=\frac{1}{2}\left\{\partial_{0} \psi_{\lambda} \partial_{0} \psi_{\lambda}^{\dagger}+\partial_{0} \phi_{\sigma} \partial_{0} \phi_{\sigma}^{\dagger}+\partial_{0} \Phi \partial_{0} \Phi^{\dagger}+\frac{1}{2} \partial_{0} \Lambda \partial_{0} \Lambda^{\dagger}+\partial_{0} \eta_{\sigma} \partial_{0} \eta_{\sigma}^{\dagger}\right. \\
& +\partial_{0} \lambda \partial_{0} \lambda^{\dagger}-p^{2} \psi_{\lambda} \psi_{\lambda}^{\dagger}-\frac{p^{2}}{2} \phi_{\sigma} \phi_{\sigma}^{\dagger}-p^{2} \Phi \Phi^{\dagger}-\frac{p^{2}}{2} \eta_{\sigma} \eta_{\sigma}^{\dagger}-p^{2} \lambda \lambda^{\dagger} \\
& +\frac{p^{2}}{2} e^{\gamma \sigma}\left(\eta_{\sigma} \phi_{\gamma}^{\dagger}+\phi_{\gamma} \eta_{\sigma}^{\dagger}\right)-i g p U e^{\sigma \gamma} \eta_{\sigma} \eta_{\gamma}^{\dagger}+i g p U Q^{\lambda \gamma} \psi_{\lambda} \psi_{\gamma}^{\dagger} \\
& +i g p U e^{\sigma \gamma} \phi_{\sigma} \phi_{\gamma}^{\dagger}+i g p U\left(2 \Phi \lambda^{\dagger}-2 \lambda \Phi^{\dagger}+\Lambda \lambda^{\dagger}-\lambda \Lambda^{\dagger}\right) \\
& \left.-2 g^{2} U^{2} \eta_{\sigma} \eta_{\sigma}^{\dagger}-2 g^{2} U^{2} \lambda \lambda^{\dagger}-g^{2} U^{2}\left(4 \Phi \Phi^{\dagger}+2 \Phi \Lambda^{\dagger}+2 \Lambda \Phi^{\dagger}+\Lambda \Lambda^{\dagger}\right)\right\}, \\
& \mathcal{H}_{\mathrm{YM}}^{\text {waves }}=\frac{1}{2}\left\{\partial_{0} \psi_{\lambda} \partial_{0} \psi_{\lambda}^{\dagger}+\partial_{0} \phi_{\sigma} \partial_{0} \phi_{\sigma}^{\dagger}+\partial_{0} \Phi \partial_{0} \Phi^{\dagger}+\frac{1}{2} \partial_{0} \Lambda \partial_{0} \Lambda^{\dagger}+\partial_{0} \eta_{\sigma} \partial_{0} \eta_{\sigma}^{\dagger}\right. \\
& +\partial_{0} \lambda \partial_{0} \lambda^{\dagger}+p^{2} \psi_{\lambda} \psi_{\lambda}^{\dagger}+\frac{p^{2}}{2} \phi_{\sigma} \phi_{\sigma}^{\dagger}+p^{2} \Phi \Phi^{\dagger}+\frac{p^{2}}{2} \eta_{\sigma} \eta_{\sigma}^{\dagger}+p^{2} \lambda \lambda^{\dagger} \\
& -\frac{p^{2}}{2} e^{\gamma \sigma}\left(\eta_{\sigma} \phi_{\gamma}^{\dagger}+\phi_{\gamma} \eta_{\sigma}^{\dagger}\right)+i g p U e^{\sigma \gamma} \eta_{\sigma} \eta_{\gamma}^{\dagger}-i g p U Q^{\lambda \gamma} \psi_{\lambda} \psi_{\gamma}^{\dagger} \\
& -i g p U e^{\sigma \gamma} \phi_{\sigma} \phi_{\gamma}^{\dagger}-i g p U\left(2 \Phi \lambda^{\dagger}-2 \lambda \Phi^{\dagger}+\Lambda \lambda^{\dagger}-\lambda \Lambda^{\dagger}\right) \\
& \left.+2 g^{2} U^{2} \eta_{\sigma} \eta_{\sigma}^{\dagger}+2 g^{2} U^{2} \lambda \lambda^{\dagger}+g^{2} U^{2}\left(4 \Phi \Phi^{\dagger}+2 \Phi \Lambda^{\dagger}+2 \Lambda \Phi^{\dagger}+\Lambda \Lambda^{\dagger}\right)\right\} .
\end{aligned}
$$

The terms like $U^{3}, U^{3} \Phi, U^{2} \Phi$ etc do not appear in the "condensate + waves" Hamiltonian above since $U(t)$ is a spatially-homogeneous function such that any spacial derivatives of $U(t)$ which could give rise to these terms simply disappear. It is straightforward to check that the system of equations (4.13) and (4.14) can be obtained directly from eq. (4.20) or (4.21) in the usual way consistent with canonical quantisation (see appendix B). Finally, the complete effective SU(2) YM Hamiltonian density properly including the YM condensate dynamics can be represented in terms of $\mathcal{H}_{\mathrm{YM}}^{\text {waves }}(4.21)$ as follows

$$
\mathcal{H}_{\mathrm{YM}}=\mathcal{H}_{\mathrm{YMC}}+\sum_{\vec{p}} \mathcal{H}_{\mathrm{YM}}^{\text {waves }}
$$

which will be used below in studies of the dynamical properties of the "waves + condensate" system below. 


\subsubsection{Longitudinal Yang-Mills wave modes: free vs interacting case}

To start with, let us consider the limiting case of free YM field without taking into account its interactions with the YM condensate, i.e. setting $U=0$ in eqs. (4.13) and (4.14). In this case we have the following reduced system

$$
\begin{aligned}
& \partial_{0} \partial_{0} \Lambda=0, \quad \partial_{0} \partial_{0} \Phi+p^{2} \Phi=0, \quad \partial_{0} \partial_{0} \lambda+p^{2} \lambda=0, \quad \partial_{0} \partial_{0} \psi_{\lambda}+p^{2} \psi_{\lambda}=0, \\
& \partial_{0} \partial_{0} \phi_{\sigma}+\frac{p^{2}}{2} \phi_{\sigma}-\frac{p^{2}}{2} e^{\sigma \gamma} \eta_{\gamma}=0, \quad \partial_{0} \partial_{0} \eta_{\sigma}+\frac{p^{2}}{2} \eta_{\sigma}-\frac{p^{2}}{2} e^{\gamma \sigma} \phi_{\gamma}=0,
\end{aligned}
$$

and two constraints

$$
i p \partial_{0} \Lambda=0, \quad i p \partial_{0} \phi_{\gamma}+i p e^{\gamma \sigma} \partial_{0} \eta_{\sigma}=0,
$$

since the considered case with $U=0$ corresponds to a degenerate YM theory. Notably, now the constraints allow to eliminate three d.o.f., namely, $\Lambda$ and $\eta_{\alpha}$, which can be associated with three unphysical longitudinal polarisations of free gauge field $A_{\mu}^{a}$ such that

$$
\Lambda=0, \quad \eta_{\alpha}=e^{\alpha \beta} \phi_{\beta} .
$$

Thus, in the considering limiting case the constraints reduce the number of physical d.o.f.'s from nine down to six transverse ones. Note, such a reduction is not possible for non-zeroth interactions with the YM condensate, e.g. when $U \neq 0$ in eqs. (4.13) and (4.14). This is because in the general case the constraints (4.18) and (4.19) cannot be represented in the form of motion integrals as it used to take place in the standard case without the YM condensate such that the longitudinal d.o.f.'s cannot be eliminated anymore. This fact essentially means that interactions of the wave modes with the homogeneous YM condensate dynamically generate three additional d.o.f.'s $\Lambda$ and $\eta_{\alpha}$, such that both the longitudinal and transverse polarisations of interacting YM field have the status of physical d.o.f.'s and therefore must be treated on the same footing. The latter statement is in a good agreement with the canonical quantisation procedure (see appendices A and B) and is confirmed by numerical analysis of the complete system (4.13) and (4.14).

The effect of dynamical generation of the longitudinal modes in a YM medium and their dynamical role has been previously discussed in the literature (see e.g. ref. [24]). Noticeably enough, it turns out that even in the simple linearized model considered above the longitudinal modes acquire proper frequencies due to YM condensate-wave interactions. As long as one incorporates the homogeneous condensate in the initial YM Lagrangian, the canonical quantisation of longitudinal modes appears to be natural and theoretically consistent without introducing any "virtual" infinitesimal terms as in the degenerate case discussed in section 2.1 (see also appendix A). Physically, it means that the longitudinal (plasma) waves in the condensate get excited together with transverse ones and the both contribute to observable quantities and should be studied on the same footing.

\subsubsection{Evolution of the Yang-Mills wave modes}

Now, consider dynamics of the wave modes in the linear approximation given by the system of YM equations (4.1), (4.13) and (4.14) without taking into account "back reaction" effects of the wave modes to the YM condensate. In fact, eq. (4.13) is a closed system of 

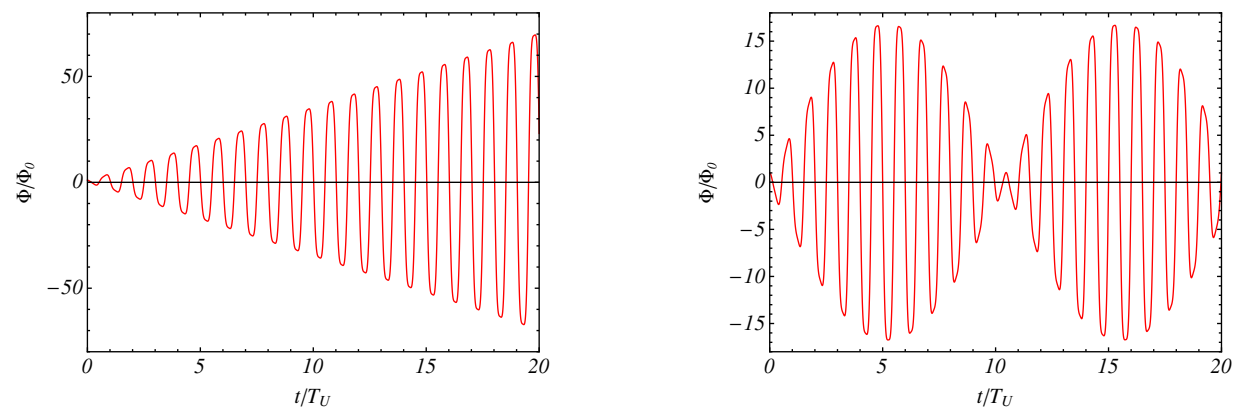

Figure 2. An example of normalized numerical solution of the system of YM equations (4.1), (4.13) and (4.14) for one of the wave modes, $\Phi=\Phi(t)$, in the case of monotonic growth of oscillations for $p=0$ (left), and in the resonance-like instability domain with harmonic impulses for a particular value $p=0.1 g U_{0}$ (right). Here and below, the initial values of all the wave modes $\left(\Phi_{0}, \lambda_{0}, \ldots\right)$ are fixed to be small in comparison to the amplitude of YM condensate oscillations.

two equations for two functions $\psi_{k}, k=1,2$, and thus can be analysed separately. By an appropriate choice of the frame of reference, the matrices $Q_{i j}^{\lambda}$ and vector $n_{i}$ can be represented in the following simple form

$$
Q_{i j}^{\lambda=1}=\left(\begin{array}{ccc}
1 & 0 & 0 \\
0 & -1 & 0 \\
0 & 0 & 0
\end{array}\right), \quad Q_{i j}^{\lambda=2}=\left(\begin{array}{lll}
0 & 1 & 0 \\
1 & 0 & 0 \\
0 & 0 & 0
\end{array}\right), \quad n_{i}=(0,0,1) .
$$

Further, introducing superpositions

$$
\psi_{1} \equiv \psi_{1}^{\prime}+\psi_{2}^{\prime}, \quad \psi_{2} \equiv i\left(\psi_{1}^{\prime}-\psi_{2}^{\prime}\right)
$$

equation (4.13) in the above basis falls apart into two independent equations for $\psi_{1,2}^{\prime}$

$$
\begin{aligned}
& \partial_{0} \partial_{0} \psi_{1}^{\prime}+\left(p^{2}+2 g p U\right) \psi_{1}^{\prime}=0, \\
& \partial_{0} \partial_{0} \psi_{2}^{\prime}+\left(p^{2}-2 g p U\right) \psi_{2}^{\prime}=0,
\end{aligned}
$$

which are recognized as Mathieu equations. The above transformation (4.24) has a direct analogy with transition from linearly polarised modes to circularly polarised ones, and is very convenient in the current setting. Above, $U=U(t)$ is a solution of YM condensate equation (4.3). Notably, the parametric resonance (or instability) domains are well known for this type of equations. In particular, for the tensor $\psi_{k}$ mode the first such parametric resonance instability domain can be found approximately as $0.15 g U_{0} \lesssim p \lesssim 4.55 g U_{0}$, where $U_{0} \equiv U\left(t=t_{0}\right)$ is an initial value of $\mathrm{YM}$ condensate. Other wave modes have different resonance-like instability domains which can be found numerically.

An analytical analysis of remaining equations (4.14) is less feasible due to the presence of quadratic term in YM condensate, $\sim U^{2}$. In addition, our numerical study has shown that one should not use the approximation (4.3) in this case such that dynamics of the wave modes becomes very different from the well-known picture of parametric resonance in the case of Mathieu equations. Nevertheless, exact numerical analysis of the complete system of equations reveals the existence of the resonance-like instability domains for all 
of the wave d.o.f. analogical to the rigorous parametric (Mathieu) resonance one of the $\psi_{k}$ mode.

As an example, in figure 2 we represent the normalised numerical solution for one of the wave modes, $\Phi=\Phi(t)$, for two distinct cases: a solution with monotonically growing amplitude for $p=0$ (left) and a solution from the parametric resonance-type instability domain with harmonic impulses for a particular value of momentum $p=0.1 g U_{0}$ (right). The observed growth of $\Phi$ amplitude or, equivalently, its energy is triggered by its interactions with the YM condensate. The same effect has been observed for all other modes as well. We thus conclude that the particles energy dynamically increases in the course of time evolution of "particles + condensate" system due to parametric resonance-like instability of numerical solutions of the non-linear YM equations.

Note, in all the numerical calculations the initial values of all the wave modes $\left(\Phi_{0}, \lambda_{0}, \ldots\right)$ are fixed to be small in comparison to the amplitude of YM condensate oscillations. This is in order to be consistent with the quasi-classical approximation. Under this key assumption the characteristic behaviour of all the numerical solutions in terms of the dimensionless quantities does not strongly depend on actual numerical values for the initial fields used in the calculations.

\section{3 "Back reaction" of the waves to the condensate}

Due to energy conservation the growth of energy of the wave modes observed in the previous section has to be followed by a certain redistribution of energy between YM condensate and wave modes. In order to take into account this effect consistently it is necessary to incorporate second-order contributions to the YM condensate equation for $U=U(t)(4.1)$ which account for interactions between condensate and particles as follows

$$
\begin{aligned}
\partial_{0} \partial_{0} U+2 g^{2} U^{3}+ & \frac{g^{2}}{6} U \sum_{\vec{p}}\left\langle 2 \eta_{\sigma} \eta_{\sigma}^{\dagger}+2 \lambda \lambda^{\dagger}+4 \Phi \Phi^{\dagger}+\Lambda \Lambda^{\dagger}+2 \Phi \Lambda^{\dagger}\right. \\
& \left.+2 \Lambda \Phi^{\dagger}+2 \eta_{\sigma}^{\dagger} \eta_{\sigma}+2 \lambda^{\dagger} \lambda+4 \Phi^{\dagger} \Phi+\Lambda^{\dagger} \Lambda+2 \Phi^{\dagger} \Lambda+2 \Lambda^{\dagger} \Phi\right\rangle \\
+\frac{i g}{12} \sum_{\vec{p}} p\left\langle 2 \Lambda^{\dagger} \lambda\right. & -2 \Lambda \lambda^{\dagger}+2 \Phi^{\dagger} \lambda-2 \Phi \lambda^{\dagger}+2 \lambda \Phi^{\dagger}-2 \lambda^{\dagger} \Phi \\
& -Q^{\lambda \sigma}\left(\psi_{\lambda} \psi_{\sigma}^{\dagger}-\psi_{\lambda}^{\dagger} \psi_{\sigma}\right)-e^{\sigma \gamma}\left(\phi_{\sigma} \phi_{\gamma}^{\dagger}-\phi_{\sigma}^{\dagger} \phi_{\gamma}\right)+\phi_{\sigma}^{\dagger} \eta_{\sigma}-\phi_{\sigma} \eta_{\sigma}^{\dagger} \\
& \left.+\eta_{\sigma}^{\dagger} \phi_{\sigma}-\eta_{\sigma} \phi_{\sigma}^{\dagger}+e^{\sigma \gamma}\left(\eta_{\sigma} \eta_{\gamma}^{\dagger}-\eta_{\sigma}^{\dagger} \eta_{\gamma}\right)\right\rangle=0 .
\end{aligned}
$$

Here, the averaging $\langle\ldots\rangle$ is performed over the Heisenberg vector state. Since equal wave modes with different momenta do not interact with each other then all their products disappear upon the averaging, so only products of different (interacting) modes remain. Besides, all linear and cubic terms in waves also disappear in the considering next-to-linear approximation as well.

The effective second-order Hamiltonian density incorporating the "back reaction" effect of the wave modes to the YM condensate can be represented as a sum of three components corresponding to the free YM condensate, $\mathcal{H}_{\mathrm{U}}$, free wave modes (or particles), $\mathcal{H}_{\text {particles }}$, 

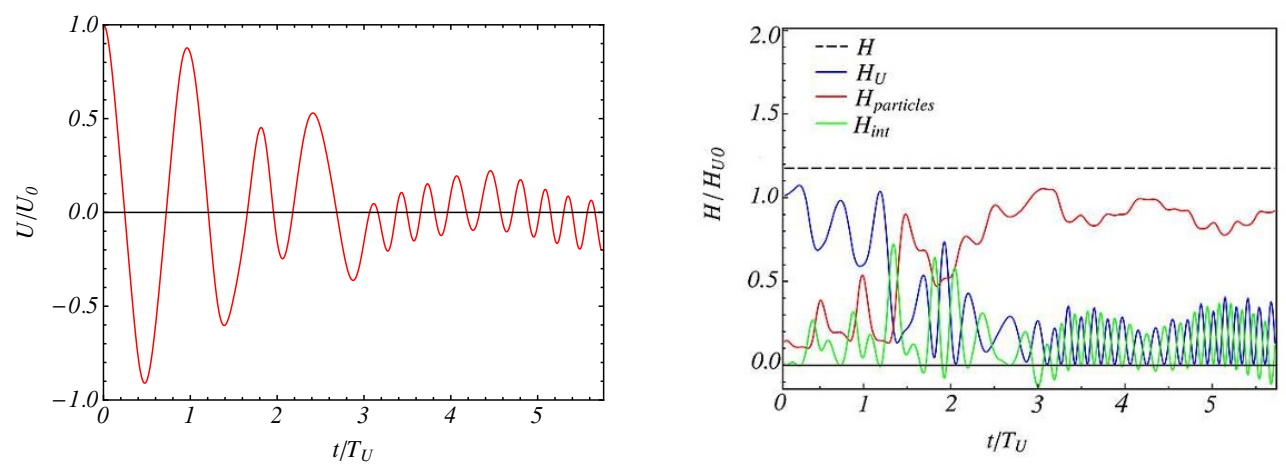

Figure 3. Time dependence of the YM condensate in the quasilinear approximation in the complete system of $\mathrm{SU}(2)$ wave modes (left), and the evolution of condensate $\mathcal{H}_{\mathrm{U}}, \mathrm{YM}$ wave modes (or particles) $\mathcal{H}_{\text {particles }}$, and interaction term $\mathcal{H}_{\text {int }}$ contributions to the total energy in the complete "condensate + waves" system (right). The illustrated numerical solutions are realistic in the quasiclassical limit of relatively small wave amplitudes, i.e. when $\left|\widetilde{A}_{i k}\right| \ll|U|$ corresponding to $t / T_{\mathrm{U}} \lesssim 1$. On the right panel, $\mathcal{H}_{U_{0}}$ is the initial energy of the YM condensate which is taken to be strongly dominating over the initial values of $\mathcal{H}_{\text {particles }}$ and $\mathcal{H}_{\text {int }}$ terms.

and the term accounting for interactions between these two subsystems, $\mathcal{H}_{\text {int }}$, respectively. The corresponding terms in the total effective Hamiltonian density $\mathcal{H}_{\text {YM }}$ given by eq. (4.22) are as follows

$$
\begin{aligned}
& \mathcal{H}_{\mathrm{U}}= \frac{3}{2}\left(\partial_{0} U \partial_{0} U+g^{2} U^{4}\right), \\
& \mathcal{H}_{\text {particles }}= \frac{1}{2} \sum_{\vec{p}}\left(\partial_{0} \psi_{\lambda} \partial_{0} \psi_{\lambda}^{\dagger}+\partial_{0} \phi_{\sigma} \partial_{0} \phi_{\sigma}^{\dagger}+\partial_{0} \Phi \partial_{0} \Phi^{\dagger}+\frac{1}{2} \partial_{0} \Lambda \partial_{0} \Lambda^{\dagger}+\partial_{0} \eta_{\sigma} \partial_{0} \eta_{\sigma}^{\dagger}\right. \\
&+\partial_{0} \lambda \partial_{0} \lambda^{\dagger}+p^{2} \psi_{\lambda} \psi_{\lambda}^{\dagger}+\frac{p^{2}}{2} \phi_{\sigma} \phi_{\sigma}^{\dagger}+p^{2} \Phi \Phi^{\dagger}+\frac{p^{2}}{2} \eta_{\sigma} \eta_{\sigma}^{\dagger}+p^{2} \lambda \lambda^{\dagger} \\
&\left.-\frac{p^{2}}{2} e^{\gamma \sigma}\left(\eta_{\sigma} \phi_{\gamma}^{\dagger}+\phi_{\gamma} \eta_{\sigma}^{\dagger}\right)\right) \\
& \mathcal{H}_{\text {int }}=\frac{1}{2} \sum_{\vec{p}}\left[\begin{array}{l}
i g p U e^{\sigma \gamma} \eta_{\sigma} \eta_{\gamma}^{\dagger}-i g p U Q^{\lambda \gamma} \psi_{\lambda} \psi_{\gamma}^{\dagger} \\
-i g p U e^{\sigma \gamma} \phi_{\sigma} \phi_{\gamma}^{\dagger}-i g p U\left(2 \Phi \lambda^{\dagger}-2 \lambda \Phi^{\dagger}+\Lambda \lambda^{\dagger}-\lambda \Lambda^{\dagger}\right) \\
\left.+2 g^{2} U^{2} \eta_{\sigma} \eta_{\sigma}^{\dagger}+2 g^{2} U^{2} \lambda \lambda^{\dagger}+g^{2} U^{2}\left(4 \Phi \Phi^{\dagger}+2 \Phi \Lambda^{\dagger}+2 \Lambda \Phi^{\dagger}+\Lambda \Lambda^{\dagger}\right)\right] .
\end{array}\right.
\end{aligned}
$$

It can be seen from these expressions that interaction term $\mathcal{H}_{\text {int }}$ is not sign-definite in distinction to positively-definite condensate $\mathcal{H}_{\mathrm{U}}$ and waves $\mathcal{H}_{\text {particles }}$ contributions.

In our numerical analysis and in all the plots in this paper we consider the complete system of all nine wave d.o.f. and YM condensate including interactions between them. We found that wave-condensate interactions lead to a decrease of amplitude of the YM condensate oscillations in time as is seen in figure 3 (left). An analogical picture of damping of the condensate oscillations is observed in the reduced (closed) system of $\Phi, \Lambda$ and $\lambda$ wave modes and the YM condensate. We have also calculated the energy evolution of particles and condensate shown in figure 3 (right). These plots clearly illustrate the energy transfer (swap) effect from the YM condensate to particles due to interactions between them. 


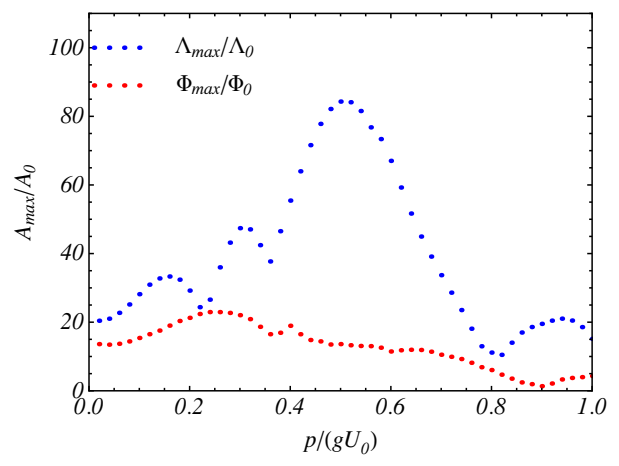

Figure 4. The particle momentum dependence of the ratio of wave amplitude (maximum over the period of oscillations) at a fixed final $t>t_{0}$ (when most of the energy of the system is concentrated in waves) to the corresponding initial amplitude at $t=t_{0}$ (when all energy of the system is concentrated in the YM condensate). The results are shown for transverse $\Phi$ and longitudinal $\Lambda$ modes.

One of the important issues in the YM theory relates to investigation of energy redistribution is the momentum spectrum of YM waves as a result of plasma self-interactions [2830]. Interactions between the YM condensate and wave modes (particles) lead to a redistribution of energy between the modes with different impulses which rather strongly depends on particles momentum due to parametric resonance-like instability of YM solutions. The latter happens because the interaction strength, and hence the energy transfer rate, depends on the amplitude of impulses which is different for different modes and particles momenta (for a given mode).

As an illustration of this effect, in figure 4 we show the particle momentum dependence (in dimensionless units) of the ratio of wave amplitude maximal over the period of oscillations at a fixed final $t>t_{0}$ to the corresponding initial amplitude at $t=t_{0}$ (when all energy of the system is concentrated in the YM condensate). The results are shown for transverse $\Phi$ and longitudinal $\Lambda$ modes and are qualitatively the same for all the other modes. We notice that an increase in wave amplitude due to parametric instability may be rather strong close to the peak regions in corresponding energy spectra. One therefore observes the ultra-relativistic particles production effect with momenta close to the resonant momenta $p \sim g U_{0}$ in a vicinity of maxima points in figure 4 .

As the main physical result to be emphasised here, we have found the significant energy swap effect between the YM condensate and particle-like modes of the ultra-relativistic YM plasma due to their interactions in quasilinear approximation. Due to energy conservation it is clear that the parametric resonance for the $\psi_{k}$ mode or a resonance-like instability for other modes in general is accompanied by an energy flow from the YM condensate to the waves. So the resonance-like instability of the quantum-wave solutions in the classical condensate is the physical reason of the energy swap effect. Note, that in figure 3 (right) we should restrict ourselves to maximal time scales of about one period of YM condensate fluctuations, $t / T_{\mathrm{U}} \lesssim 1$. At larger time scales $t / T_{\mathrm{U}}>1$ the amplitude of the wave fluctuations becomes comparable to the amplitude of condensate fluctuations so the considering quasilinear approximation breaks down there. Let us study sensitivity of our solutions with respect to higher-order corrections in detail. 


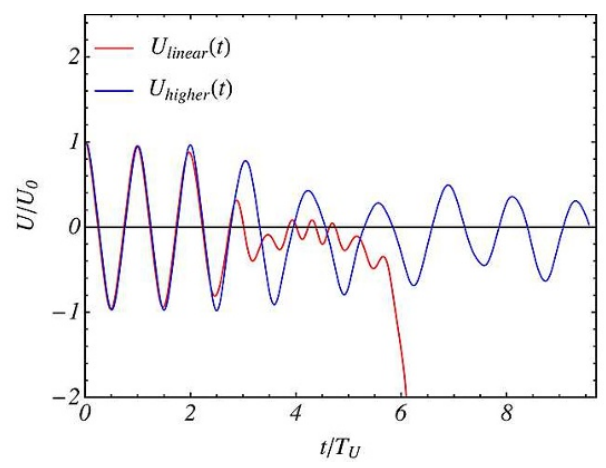

Figure 5. Time evolution of the YM condensate in the complete next-to-linear problem given by a numerical solution of eqs. (4.13), (4.14) and (4.25) (red line) and in the problem with extra higher order terms included (4.30) (blue line).

\subsection{Higher-order corrections}

So far we have considered the SU(2) YM wave dynamics in the classical YM condensate in the first (leading or linear) approximation, while the YM condensate dynamics — in the linear and next-to-linear (or quasi-linear) approximations. The range of applicability of our quasi-classical analysis is limited to small quantum-wave fluctuations $\widetilde{A}_{i k}$ in the condensate $U$ considered as a classical background, i.e. in the $\widetilde{A}_{i k} \ll U$ asymptotics.

It has been demonstrated above that the interactions between the wave modes and the condensate lead to an increase of energy accumulated by the wave modes at expense of a corresponding decrease of YM condensate energy. This means that as some point in evolution of the system the amplitude of wave modes becomes too large so that the initial approximation $\widetilde{A}_{i k} \ll U$ breaks down. Such a breakdown can be noticed in numerical results in the next-to-linear approximation, e.g. in YM condensate dynamics given by a numerical solution of eqs. (4.13), (4.14) and (4.25) illustrated in figure 5 by red line. It is clearly seen from this figure that at relatively large time scales increasing the time domain of effective energy swap from the condensate to waves, the YM condensate energy becomes singular and unbounded. The latter anomaly is due to breakdown of the nextto-linear approximation. In what follows, we show that inclusion of the principal part of the higher-order terms into the linear equations (4.13) and (4.14) for the wave modes allows to eliminate such anomalies and reveals qualitative stability of the energy swap effect under consideration.

The equations of motion for the wave $\widetilde{A}_{i k}$ modes in a given order are normally constructed after elimination of the equation of motion for the YM condensate from generic eq. (4.7). Denote the left side of eq. (4.7) as $Y_{l k}$ such that equations for the $\widetilde{A}_{l k}$ modes take the following form

$$
\widetilde{Y}_{l k}=Y_{l k}-\left\langle Y_{l k}\right\rangle=0
$$

where $\langle\ldots\rangle$ denotes averaging over the Heisenberg state vector as usual. For simplicity, we take into account the last two terms in eq. (4.7) only. Omitting all other higher-order 
terms in eq. (4.26) we have

$$
\begin{aligned}
& \partial_{0} \partial_{0} \widetilde{A}_{l k}-\partial_{i} \partial_{i} \widetilde{A}_{l k}+\partial_{i} \partial_{k} \widetilde{A}_{l i} \\
& +g e_{l m k} \partial_{i} \widetilde{A}_{m i} U+2 g e_{l i p} \partial_{i} \widetilde{A}_{p k} U+g e_{l m i} \partial_{k} \widetilde{A}_{m i} U \\
& -g^{2} \widetilde{A}_{k l} U^{2}+g^{2} \widetilde{A}_{l k} U^{2}+2 g^{2} \delta_{l k} \widetilde{A}_{i i} U^{2}+g^{2}\left(\widetilde{A}_{p i} \widetilde{A}_{p i} \widetilde{A}_{l k}-\widetilde{A}_{l i} \widetilde{A}_{p k} \widetilde{A}_{p i}\right)+\cdots=0 .
\end{aligned}
$$

Now let us multiply $\widetilde{Y}_{l k}(x, t)$ by $\widetilde{A}_{l k}$ in a different space-time point $\left(x^{\prime}, t^{\prime}\right)$ in order to make the terms of the fourth order such that

$$
\left\langle\widetilde{A}_{l k}\left(x^{\prime}, t^{\prime}\right) \widetilde{Y}_{l k}(x, t)\right\rangle=0,
$$

due to eq. (4.26). Here, each fourth-order term can then be transformed as follows

$$
\left\langle A_{1} A_{2} A_{3} A_{4}\right\rangle=\frac{1}{3}\left[\left\langle A_{1} A_{2}\right\rangle\left\langle A_{3} A_{4}\right\rangle+\left\langle A_{1} A_{3}\right\rangle\left\langle A_{2} A_{4}\right\rangle+\left\langle A_{1} A_{4}\right\rangle\left\langle A_{2} A_{3}\right\rangle\right] .
$$

Dropping $\widetilde{A}_{l k}\left(x^{\prime}, t^{\prime}\right)$ function and performing Fourier transform of the remaining terms we obtain

$$
\begin{aligned}
& \partial_{0} \partial_{0} \widetilde{A}_{l k}+p^{2} \widetilde{A}_{l k}-p^{2} n_{i} n_{k} \widetilde{A}_{l i}+i g p e_{l m k} n_{i} \widetilde{A}_{m i} U \\
& +2 i g p e_{l i p} n_{i} \partial_{i} \widetilde{A}_{p k} U+i g p e_{l m i} n_{k} \widetilde{A}_{m i} U-g^{2} \widetilde{A}_{k l} U^{2} \\
& +g^{2} \widetilde{A}_{l k} U^{2}+2 g^{2} \delta_{l k} \widetilde{A}_{i i} U^{2}-\frac{g^{2}}{12}\left[\widetilde{A}_{l i} \sum_{\vec{p}}\left\langle\widetilde{A}_{p i} \widetilde{A}_{p k}^{\dagger}+\widetilde{A}_{p i}^{\dagger} \widetilde{A}_{p k}\right\rangle\right. \\
& +\widetilde{A}_{p k} \sum_{\vec{p}}\left\langle\widetilde{A}_{l i} \widetilde{A}_{p i}^{\dagger}+\widetilde{A}_{l i}^{\dagger} \widetilde{A}_{p i}\right\rangle+\widetilde{A}_{p i} \sum_{\vec{p}}\left\langle\widetilde{A}_{l i} \widetilde{A}_{p k}^{\dagger}+\widetilde{A}_{l i}^{\dagger} \widetilde{A}_{p k}\right\rangle \\
& \left.\quad-2 \widetilde{A}_{p i} \sum_{\vec{p}}\left\langle\widetilde{A}_{p i} \widetilde{A}_{l k}^{\dagger}+\widetilde{A}_{p i}^{\dagger} \widetilde{A}_{l k}\right\rangle-\widetilde{A}_{l k} \sum_{\vec{p}}\left\langle\widetilde{A}_{p i} \widetilde{A}_{p i}^{\dagger}+\widetilde{A}_{p i}^{\dagger} \widetilde{A}_{p i}\right\rangle\right]+\cdots=0 .
\end{aligned}
$$

We notice here that the third-order terms have transformed to effective mass terms given by averages of various two operator products. In particular, figure 5 (blue line) illustrates that such higher-order effective mass terms eliminate formal singularities in the YM condensate such that the numerical solution stabilises and the energy swap effect discussed above remains at the qualitative level.

Certainly, this simplified analysis is not complete and is aimed only at illustrating that inclusion of the major part of higher-order terms significantly improves and stabilises the results of the next-to-linear model. In a fully consistent model one has to take into account contributions from all the higher-order terms both in the wave equations of motion and in the YM condensate equation simultaneously, which will be done elsewhere.

\section{Dynamics of the Yang-Mills plasma}

So far we have considered either pure condensate limit or interacting system "condensate + waves" in the small wave approximation. Now, it is worth to analyse another important 
limiting case of pure Yang-Mills (gluon) plasma composed of wave excitations only which corresponds to the case of vanishingly small condensate and large waves, i.e. $|U(t)| \ll\left|\widetilde{A}_{i k}\right|$.

The basic equation which describes dynamics of the pure gluon plasma in $\mathrm{SU}(3)$ theory has the following form

$$
\begin{aligned}
& \partial_{0} \partial_{0} \widetilde{A}_{a i}-\partial_{k} \partial_{k} \widetilde{A}_{a i}+\partial_{i} \partial_{k} \widetilde{A}_{a k} \\
& +2 g f_{a b c} \partial_{k} \widetilde{A}_{c i} \widetilde{A}_{b k}+g f_{a b c} \partial_{i} \widetilde{A}_{b k} \widetilde{A}_{c k}+g f_{a b c} \partial_{k} \widetilde{A}_{b k} \widetilde{A}_{c i} \\
& +g^{2} f_{c b a} f_{c b^{\prime} c^{\prime}} \widetilde{A}_{b k} \widetilde{A}_{b^{\prime} k} \widetilde{A}_{c^{\prime} i}=0,
\end{aligned}
$$

where $a=1, \ldots 8$. Multiplying this equation by $\widetilde{A}_{a^{\prime} i^{\prime}}\left(\overrightarrow{x^{\prime}}, t^{\prime}\right)$ and averaging the result over the state vector we obtain

$$
\begin{aligned}
& \left\langle\widetilde { A } _ { a ^ { \prime } i ^ { \prime } } ( \vec { x ^ { \prime } } , t ^ { \prime } ) \left(\partial_{0} \partial_{0} \widetilde{A}_{a i}(\vec{x}, t)-\partial_{k} \partial_{k} \widetilde{A}_{a i}(\vec{x}, t)+\partial_{i} \partial_{k} \widetilde{A}_{a k}(\vec{x}, t)\right.\right. \\
& +2 g f_{a b c} \partial_{k} \widetilde{A}_{c i}(\vec{x}, t) \widetilde{A}_{b k}(\vec{x}, t)+g f_{a b c} \partial_{i} \widetilde{A}_{b k}(\vec{x}, t) \widetilde{A}_{c k}(\vec{x}, t)+g f_{a b c} \partial_{k} \widetilde{A}_{b k}(\vec{x}, t) \widetilde{A}_{c i}(\vec{x}, t) \quad(5.2) \\
& \left.\quad+g^{2} f_{c b a} f_{c b^{\prime} c^{\prime}}\left(\widetilde{A}_{b k}(\vec{x}, t) m_{c^{\prime} i}^{b^{\prime} k}(t)+\widetilde{A}_{b^{\prime} k}(\vec{x}, t) m_{c^{\prime} i}^{b k}(t)+\widetilde{A}_{c^{\prime} i}(\vec{x}, t) m_{b^{\prime} k}^{b k}(t)\right)\right\rangle=0,
\end{aligned}
$$

where the effective mass matricies

$$
m_{b^{\prime} k^{\prime}}^{b k} \equiv \frac{1}{3}\left\langle\widetilde{A}_{b k} \widetilde{A}_{b^{\prime} k^{\prime}}\right\rangle=\frac{1}{12} \sum_{\vec{p}}\left\langle\widetilde{A}_{b k}^{r \vec{p}} \widetilde{A}_{b^{\prime} k^{\prime}}^{\dagger r \vec{p}}+\widetilde{A}_{b k}^{\dagger r \vec{p}} \widetilde{A}_{b^{\prime} k^{\prime}}^{r \vec{p}}\right\rangle .
$$

In the derivation of effective mass terms we implied that the fourth-order terms resulted from third-order ones in eq. (5.1) are transformed according to eq. (4.29). Dropping $\widetilde{A}_{a^{\prime} i^{\prime}}\left(\overrightarrow{x^{\prime}}, t^{\prime}\right)$ in eq. (5.2) we obtain

$$
\begin{aligned}
& \partial_{0} \partial_{0} \widetilde{A}_{a i}-\partial_{k} \partial_{k} \widetilde{A}_{a i}+\partial_{i} \partial_{k} \widetilde{A}_{a k} \\
& +2 g f_{a b c} \partial_{k} \widetilde{A}_{c i} \widetilde{A}_{b k}+g f_{a b c} \partial_{i} \widetilde{A}_{b k} \widetilde{A}_{c k}+g f_{a b c} \partial_{k} \widetilde{A}_{b k} \widetilde{A}_{c i} \\
& \quad+g^{2} f_{c b a} f_{c b^{\prime} c^{\prime}}\left(\widetilde{A}_{b k} m_{c^{\prime} i}^{b^{\prime} k}+\widetilde{A}_{b^{\prime} k} m_{c^{\prime} i}^{b k}+\widetilde{A}_{c^{\prime} i} m_{b^{\prime} k}^{b k}\right)=0 .
\end{aligned}
$$

\subsection{Effective gluon mass from wave self-interactions}

For simplicity, at this very first step here we would like to investigate the influence of the odd-power terms in waves, i.e. the first- and third-order terms in eq. (5.4) only. Let us divide eq. (5.4) into two equations for transverse and longitudinal modes using $\widetilde{A}_{a i}=\widetilde{A}_{a i \perp}+\widetilde{A}_{a i \|}$. For this purpose, one multiplies eq. (5.4) by $\widetilde{A}_{a^{\prime} i^{\prime} \perp}$ and $\widetilde{A}_{a^{\prime} i^{\prime} \|}$ and then averages over the state vector. For simplicity, the contributions from second order terms in eq. (5.1) can be omitted since being multiplied by $\widetilde{A}_{a^{\prime} i^{\prime} \perp}$ they are expected to be small upon averaging over the state vector in the "soft" limit $p \rightarrow 0$ in the beginning of time evolution. While this toy-model is very useful for understanding of generic features of the gluon plasma, in the realistic plasma case one should go beyond this approximation (see below).

Finally, one notices that the equation of motion for the transversely-polarised gluon plasma modes takes the form of the Klein-Gordon-Fock equation

$$
\partial_{0} \partial_{0} \widetilde{A}_{a i \perp}-\partial_{k} \partial_{k} \widetilde{A}_{a i \perp}+m_{\perp}^{2} \widetilde{A}_{a i \perp}=0
$$

with effective mass

$$
m_{\perp}^{2}=\frac{1}{12} g^{2}\left(\left\langle\widetilde{A}_{a i \|} \widetilde{A}_{a i \|}\right\rangle+\left\langle\widetilde{A}_{a i \perp} \widetilde{A}_{a i \perp}\right\rangle\right)
$$


Note, self-interactions of the plasma modes described by the cubic terms in eq. (5.1) naturally generate constituent mass of transversely polarised gluons (5.6).

In analogy to the transverse modes, dynamics of the longitudinal modes in the pure gluon plasma is described by

$$
\partial_{0} \partial_{0} \widetilde{A}_{a i \|}+m_{\|}^{2} \widetilde{A}_{a i \|}=0
$$

where the effective mass of longitudinal modes

$$
m_{\|}^{2}=\frac{1}{12} g^{2}\left(\left\langle\widetilde{A}_{a i \|} \widetilde{A}_{a i \|}\right\rangle+\left\langle\widetilde{A}_{a i \perp} \widetilde{A}_{a i \perp}\right\rangle\right),
$$

turns out to be equal to the mass of transverse modes,

$$
m^{2} \equiv m_{\|}^{2}=m_{\perp}^{2} .
$$

Note, both transverse and longitudinal modes contribute to the effective mass (5.9) due to their mutual interactions. The dispersion relations for transverse and longitudinal modes follow directly from eqs. (5.5) and (5.7),

$$
\varepsilon_{p, \lambda}^{2}=p^{2}+m^{2}, \quad \varepsilon_{p, 0}^{2}=m^{2} .
$$

The solutions of eqs. (5.5) and (5.7) can then be found in the following form

$$
\widetilde{A}_{i}^{a}=\sum_{\vec{p}, \eta=-1,0,1} \frac{1}{\sqrt{2 V \varepsilon_{p, \eta}}} e_{\vec{p}, \eta}^{i}\left(c_{\vec{p}, \eta}^{a} e^{-i\left(\varepsilon_{p, \eta} t-\vec{p} \vec{x}\right)}+c_{\vec{p}, \eta}^{a \dagger} e^{i\left(\varepsilon_{p, \eta} t-\vec{p} \vec{x}\right)}\right),
$$

where $e_{\vec{p}, \eta}^{i}$ is the plane wave polarisation vector. Substituting this solution into eq. (5.6) one arrives at

$$
m^{2}=\frac{1}{12 V} g^{2}\left(\sum_{\vec{p}, \lambda= \pm 1} \frac{N_{\vec{p}, \lambda}}{\varepsilon_{p, \lambda}}+\sum_{\vec{p}} \frac{N_{\vec{p}, 0}}{\varepsilon_{p, 0}}\right)+m_{0}^{2}
$$

where

$$
m_{0}^{2}=\frac{2}{3 V} g^{2} \sum_{\vec{p}}\left(\frac{1}{\varepsilon_{p, \lambda}}+\frac{1}{2 \varepsilon_{p, 0}}\right) .
$$

Here, $N_{\vec{p}, \lambda}$ and $N_{\vec{p}, 0}$ should be treated as the occupation numbers of longitudinal and transverse gluons, respectively,

$$
\left[c_{\vec{p}, \eta}^{\dagger a} c_{\vec{p}^{\prime}, \eta^{\prime}}^{a^{\prime}}\right]=-\delta_{\vec{p} \vec{p}^{\prime}} \delta_{\eta \eta^{\prime}} \delta_{a a^{\prime}}, \quad \sum_{a}\left\langle c_{\vec{p}, \eta}^{\dagger a} c_{\vec{p}^{\prime}, \eta^{\prime}}^{a}\right\rangle=\delta_{\vec{p} \vec{p}^{\prime}} \delta_{\eta \eta^{\prime}} N_{\vec{p}, \eta}, \quad \eta, \eta^{\prime}=-1,0,+1
$$

Applying the dimensional regularisation technique, one can represent the divergent sums over $\vec{p}$ in refs. (5.12) and (5.13) in $1+n$ dimensions where $n=3-2 \epsilon$ according to the following example

$$
\frac{g_{n}^{2} n}{V_{n}} \sum_{|\vec{p}|} \frac{1}{\varepsilon_{p}}=\frac{n g_{n}^{2} 2 \pi^{n / 2} l^{n-3}}{(2 \pi)^{n} \Gamma(n / 2)} \int_{0}^{\infty} \frac{p^{n-1} d p}{\varepsilon_{p}}=-\frac{g_{n}^{2} m^{2} n}{4 \pi(n-1)}\left(\frac{m^{2} l^{2}}{4 \pi}\right)^{\frac{n-3}{2}} \Gamma\left(\frac{3-n}{2}\right),
$$


where the dispersion relation $\varepsilon_{p}^{2}=p^{2}+m^{2}$ is employed and the parameter $l$ has been introduced to preserve the overall dimension. Repeating the same procedure for other relevant sums and then performing $\epsilon \rightarrow 0$ expansion one obtains the following final expressions

$$
\begin{aligned}
& \frac{g_{n}^{2} n}{V_{n}} \sum_{|\vec{p}|} \frac{1}{\varepsilon_{p}}=-\frac{3 g^{2}}{8 \pi^{2}} m^{2}\left(\frac{1}{\epsilon}-\ln \frac{m^{2}}{\mu^{2}}+O(\epsilon)\right), \\
& \frac{g_{n}^{2} n}{V_{n}} \sum_{|\vec{p}|} \varepsilon_{p}=-\frac{3 g^{2}}{32 \pi^{2}} m^{4}\left(\frac{1}{\epsilon}-\ln \frac{m^{2}}{\sqrt{e} \mu^{2}}+O(\epsilon)\right), \\
& \frac{g_{n}^{2} n}{V_{n}} \sum_{|\vec{p}|} \frac{|\vec{p}|^{2}}{\varepsilon_{p}}=\frac{9 g^{2}}{32 \pi^{2}} m^{4}\left(\frac{1}{\epsilon}-\ln \frac{e^{\frac{1}{6}} m^{2}}{\mu^{2}}+O(\epsilon)\right),
\end{aligned}
$$

where the renormalization scale is defined as

$$
\mu^{2}=\frac{4 \pi e^{\frac{1}{3}-c}}{l^{2}}, \quad c=0.5772157 .
$$

In what follows we assume that all the terms proportional to $1 / \varepsilon$ in eqs. (5.15)-(5.17) are cancelled in a complete remormalizable theory and thus can be discarded.

The form of dispersion relations does not enable us to renormalized the contributions from longitudinal modes, so let us leave them in the initial form and transform the vacuum terms induced by the transverse modes only. In this way, we end up with the following expression for renormalized mass

$$
m^{2}=\frac{1}{3}\left[\frac{1}{4 V} g^{2} \sum_{\vec{p}, \lambda= \pm 1} \frac{n_{\vec{p}, \lambda}}{\varepsilon_{p, \lambda}}+\frac{1}{4 \pi^{2}} g^{2} m^{2} \ln \frac{m^{2}}{\mu^{2}}+\frac{1}{4 V} g^{2} \sum_{\vec{p}} \frac{n_{\vec{p}, 0}}{\varepsilon_{p, 0}}+\frac{1}{V} g^{2} \sum_{\vec{p}} \frac{1}{\varepsilon_{p, 0}}\right] .
$$

A validation criterion for the effective mass result (5.19) is based on a consistency of the considered quantum field theory and thermodynamic approaches. Since we considered the gluon plasma in thermodynamic equilibrium, the same expression for effective mass should be derived from the minimality condition for thermodynamic potentials. For example, such a condition for the plasma energy reads

$$
\left(\frac{\partial\langle H\rangle}{\partial m}\right)_{S}=0
$$

Then, the time independence of entropy means that the occupation numbers are constant. Under this condition the expression for the plasma energy accounting for contributions from quadratic and quartic terms only takes the final form

$$
\langle H\rangle=\sum_{\vec{p}, \lambda= \pm 1} \varepsilon_{p, \lambda} n_{\vec{p}, \lambda}+8 \sum_{\vec{p}} \varepsilon_{p, \lambda}+\sum_{\vec{p}} \varepsilon_{p, 0} n_{\vec{p}, 0}+4 \sum_{\vec{p}} \varepsilon_{p, 0}-\frac{3 m^{4} V}{g^{2}} .
$$

It is straightforward to check that by substitution of eq. (5.21) into eq. (5.20) one obtains the same expression for the effective mass as the one in eq. (5.19) thus validating the above calculations. 


\subsection{Plasma equations with second- and third-order wave self-interactions}

Our goal now is to analyse the influence of the second order terms in eq. (5.4) to the gluon plasma dynamics. It will be explicitly demonstrated that these terms also contribute to the constituent gluon mass which then appears to be non-local in time.

Consider the YM wave field $\widetilde{A}_{a i}$ as the sum of "real" and "virtual" parts

$$
\widetilde{A}_{a i}=\widetilde{A}_{a i}^{r}+\widetilde{A}_{a i}^{v}
$$

such that the "virtual" part $\left|\widetilde{A}_{a i}^{v}\right| \ll\left|\widetilde{A}_{a i}^{r}\right|$ and the coupling constant $g \ll 1$ are assumed to be small to the same order, i.e. $\left|\widetilde{A}_{a i}^{v}\right| /\left|\widetilde{A}_{a i}^{r}\right| \sim g$, for simplicity.

In the first-order approximation, the equation for the "real" field $\widetilde{A}_{a i}^{r}$ has the following form

$$
\partial_{0} \partial_{0} \widetilde{A}_{a i}^{r}-\partial_{k} \partial_{k} \widetilde{A}_{a i}^{r}+\partial_{i} \partial_{k} \widetilde{A}_{a k}^{r}=0
$$

In the second-order approximation, the "real" and "virtual" parts satisfy the following equation up to the terms proportional to $g^{2}$

$$
\begin{aligned}
\partial_{0} \partial_{0} \widetilde{A}_{a i}^{r} & +\partial_{0} \partial_{0} \widetilde{A}_{a i}^{v}-\partial_{k} \partial_{k} \widetilde{A}_{a i}^{r}-\partial_{k} \partial_{k} \widetilde{A}_{a i}^{v}+\partial_{i} \partial_{k} \widetilde{A}_{a k}^{r}+\partial_{i} \partial_{k} \widetilde{A}_{a k}^{v} \\
& +2 g f_{a b c} \partial_{k} \widetilde{A}_{c i}^{r} \widetilde{A}_{b k}^{r}+2 g f_{a b c} \partial_{k} \widetilde{A}_{c i}^{r} \widetilde{A}_{b k}^{v}+2 g f_{a b c} \partial_{k} \widetilde{A}_{c i}^{v} \widetilde{A}_{b k}^{r}+g f_{a b c} \partial_{i} \widetilde{A}_{b k}^{r} \widetilde{A}_{c k}^{r} \\
& +g f_{a b c} \partial_{i} \widetilde{A}_{b k}^{r} \widetilde{A}_{c k}^{v}+g f_{a b c} \partial_{i} \widetilde{A}_{b k}^{v} \widetilde{A}_{c k}^{r}+g f_{a b c} \partial_{k} \widetilde{A}_{b k}^{r} \widetilde{A}_{c i}^{r}+g f_{a b c} \partial_{k} \widetilde{A}_{b k}^{r} \widetilde{A}_{c i}^{v} \\
& +g f_{a b c} \partial_{k} \widetilde{A}_{b k}^{v} \widetilde{A}_{c i}^{r}+g^{2} f_{c b a} f_{c b^{\prime} c^{\prime}}\left(\widetilde{A}_{b k}^{r} m_{c^{\prime} i}^{b^{\prime} k}+\widetilde{A}_{b^{\prime} k}^{r} m_{c^{\prime} i}^{b k}+\widetilde{A}_{c^{\prime} i}^{r} m_{b^{\prime} k}^{b k}\right)
\end{aligned}
$$

where the effective mass terms $m_{b^{\prime} k^{\prime}}^{b k}$ are found in eq. (5.3).

The equation for the "virtual" part is obtained by subtracting the first-order equation for the "real" one (5.23) and keeping the terms linear in $g$ only such that

$$
\begin{aligned}
\partial_{0} \partial_{0} \widetilde{A}_{a i}^{v} & -\partial_{k} \partial_{k} \widetilde{A}_{a i}^{v}+\partial_{i} \partial_{k} \widetilde{A}_{a k}^{v} \\
& +2 g f_{a b c} \partial_{k} \widetilde{A}_{c i}^{r} \widetilde{A}_{b k}^{r}+g f_{a b c} \partial_{i} \widetilde{A}_{b k}^{r} \widetilde{A}_{c k}^{r}+g f_{a b c} \partial_{k} \widetilde{A}_{b k}^{r} \widetilde{A}_{c i}^{r}=0 .
\end{aligned}
$$

The equation for the "real" part in the second (in $g$ ) approximation is then obtained by subtracting eq. (5.25) from eq. (5.24).

One immediately notices that the "virtual" part can be expressed via square of the "real" part from eq. (5.25). Performing Fourier transform of eq. (5.25) it appears to be exactly solvable by variational methods. Corresponding solutions for the "virtual" part expressed in terms of the "real" one read

$$
\begin{aligned}
& \widetilde{A}_{a i}^{v 0}(t)= \int_{t_{0}}^{t} t^{\prime} d t^{\prime} \frac{1}{V} \int d^{3} x^{\prime} g f_{a b c}\left(2 \partial_{k} \widetilde{A}_{c i}^{r}\left(x^{\prime}\right) \widetilde{A}_{b k}^{r}\left(x^{\prime}\right)\right. \\
&\left.+\partial_{i} \widetilde{A}_{b k}^{r}\left(x^{\prime}\right) \widetilde{A}_{c k}^{r}\left(x^{\prime}\right)+\partial_{k} \widetilde{A}_{b k}^{r}\left(x^{\prime}\right) \widetilde{A}_{c i}^{r}\left(x^{\prime}\right)\right) \\
&-\int_{t_{0}}^{t} d t^{\prime} \frac{1}{V} \int d^{3} x^{\prime} g f_{a b c}\left(2 \partial_{k} \widetilde{A}_{c i}^{r}\left(x^{\prime}\right) \widetilde{A}_{b k}^{r}\left(x^{\prime}\right)\right. \\
&\left.+\partial_{i} \widetilde{A}_{b k}^{r}\left(x^{\prime}\right) \widetilde{A}_{c k}^{r}\left(x^{\prime}\right)+\partial_{k} \widetilde{A}_{b k}^{r}\left(x^{\prime}\right) \widetilde{A}_{c i}^{r}\left(x^{\prime}\right)\right) t,
\end{aligned}
$$




$$
\begin{aligned}
& \widetilde{A}_{a \|}^{v \vec{p}}(t)=\int_{t_{0}}^{t} t^{\prime} d t^{\prime} \frac{1}{V} \int d^{3} x^{\prime} g f_{a b c} e^{-i \vec{p} x^{\prime}} n_{i}( 2 \partial_{k} \widetilde{A}_{c i}^{r}\left(x^{\prime}\right) \widetilde{A}_{b k}^{r}\left(x^{\prime}\right) \\
&\left.+\partial_{i} \widetilde{A}_{b k}^{r}\left(x^{\prime}\right) \widetilde{A}_{c k}^{r}\left(x^{\prime}\right)+\partial_{k} \widetilde{A}_{b k}^{r}\left(x^{\prime}\right) \widetilde{A}_{c i}^{r}\left(x^{\prime}\right)\right) \\
&-\int_{t_{0}}^{t} d t^{\prime} \frac{1}{V} \int d^{3} x^{\prime} g f_{a b c} e^{-i \vec{p} \vec{x}^{\prime}} n_{i}\left(2 \partial_{k} \widetilde{A}_{c i}^{r}\left(x^{\prime}\right) \widetilde{A}_{b k}^{r}\left(x^{\prime}\right)\right.\left.+\partial_{i} \widetilde{A}_{b k}^{r}\left(x^{\prime}\right) \widetilde{A}_{c k}^{r}\left(x^{\prime}\right)+\partial_{k} \widetilde{A}_{b k}^{r}\left(x^{\prime}\right) \widetilde{A}_{c i}^{r}\left(x^{\prime}\right)\right) t \\
& \widetilde{A}_{a \alpha}^{v \vec{p}}(t)=-\int_{t_{0}}^{t} d t^{\prime} \frac{1}{V} \int d^{3} x^{\prime} \frac{g}{2 i p} f_{a b c} e^{-i \vec{p} x^{\prime}} e^{i p\left(t-t^{\prime}\right)} s_{i}^{\alpha}\left(2 \partial_{k} \widetilde{A}_{c i}^{r}\left(x^{\prime}\right) \widetilde{A}_{b k}^{r}\left(x^{\prime}\right)\right. \\
&\left.+\partial_{i} \widetilde{A}_{b k}^{r}\left(x^{\prime}\right) \widetilde{A}_{c k}^{r}\left(x^{\prime}\right)+\partial_{k} \widetilde{A}_{b k}^{r}\left(x^{\prime}\right) \widetilde{A}_{c i}^{r}\left(x^{\prime}\right)\right) \\
&+\int_{t_{0}}^{t} d t^{\prime} \frac{1}{V} \int d^{3} x^{\prime} \frac{g}{2 i p} f_{a b c} e^{-i \vec{p} x^{\prime}} e^{i p\left(t^{\prime}-t\right)} s_{i}^{\alpha}\left(2 \partial_{k} \widetilde{A}_{c i}^{r}\left(x^{\prime}\right) \widetilde{A}_{b k}^{r}\left(x^{\prime}\right)\right. \\
&\left.+\partial_{i} \widetilde{A}_{b k}^{r}\left(x^{\prime}\right) \widetilde{A}_{c k}^{r}\left(x^{\prime}\right)+\partial_{k} \widetilde{A}_{b k}^{r}\left(x^{\prime}\right) \widetilde{A}_{c i}^{r}\left(x^{\prime}\right)\right) .
\end{aligned}
$$

Above, $\widetilde{A}_{a \|}^{v \vec{p}}(t)$ and $\widetilde{A}_{a \alpha}^{v \vec{p}}(t)$ are the longitudinal and transverse parts of $\widetilde{A}_{a i}^{v \vec{p}}$, $\widetilde{A}_{a i}^{v 0}(t)$ is the mode with zero momentum, and $\widetilde{A}_{c k}^{r}\left(x^{\prime}\right)=\widetilde{A}_{c k}^{r}\left(t^{\prime}, \overrightarrow{x^{\prime}}\right)$. For the initial conditions at $t=t_{0}$, we adopted that $\widetilde{A}_{a \|}^{v \vec{p}}\left(t_{0}\right)=\widetilde{A}_{a \alpha}^{v \vec{p}}\left(t_{0}\right)=\widetilde{A}_{a i}^{v 0}\left(t_{0}\right)=0$ for all the polarisations and momenta. Indeed, this condition agrees well will the initial procedure of splitting a single wave mode into two parts, the large "real" and small "virtual" ones, whose initial values must be consistent.

By a substitution of eqs. (5.27) and (5.28) into eq. (5.24), the second order terms can be transformed to the third-order ones over the "real" field which then evaluated by means of the same algorithm as used above. Finally, eq. (5.24) gets transformed to

$$
\begin{aligned}
\partial_{0} \partial_{0} \widetilde{A}_{a i}^{r \vec{q}}+q^{2} \widetilde{A}_{a i}^{r \vec{q}}-q_{i} q_{k} \widetilde{A}_{a k}^{r \vec{q}}+\int_{t 0}^{t} \widetilde{A}_{c^{\prime} i^{\prime}}^{r \vec{q}}\left(t^{\prime}\right) M_{a i c^{\prime} i^{\prime}}^{\vec{q}}\left(t, t^{\prime}\right) d t^{\prime} \\
+g^{2} f_{c b a} f_{c b^{\prime} c^{\prime}}\left(\widetilde{A}_{b k}^{r \vec{q}} m_{c^{\prime} i}^{b^{\prime} k}+\widetilde{A}_{b^{\prime} k}^{r \vec{q}} m_{c^{\prime} i}^{b k}+\widetilde{A}_{c^{\prime} i}^{r \vec{q}} m_{b^{\prime} k}^{b k}\right)=0,
\end{aligned}
$$

where $m_{b k}^{a i}$ are the contributions from third-order terms in wave modes given by eq. (5.3), and the second-order contribution reads

$$
\begin{aligned}
M_{a i c^{\prime} i^{\prime}}^{\vec{q}}\left(t, t^{\prime}\right) & \\
= & -\frac{1}{8} \sum_{\vec{p}}\left[\left(\hat{a}_{\vec{q}-\vec{p}}^{a c b^{\prime} i^{\prime} i^{\prime} k}\left(2 q_{k^{\prime}}-p_{k^{\prime}}\right)\left(q_{k}+p_{k}\right)\right.\right. \\
& \left.+\hat{a}_{\vec{q}-\vec{p}}^{a c c^{\prime} k^{\prime} k^{\prime} k}\left(q_{i^{\prime}}-2 p_{i^{\prime}}\right)\left(q_{k}+p_{k}\right)\right)\left\langle\widetilde{A}_{c i}^{r \vec{p}}(t) \widetilde{A}_{b^{\prime} k^{\prime}}^{\dagger r \vec{p}}\left(t^{\prime}\right)\right\rangle \\
& +\left(\hat{a}_{\vec{q}+\vec{p}}^{a c b^{\prime} i^{\prime} i^{\prime} k}\left(2 q_{k^{\prime}}+p_{k^{\prime}}\right)\left(q_{k}-p_{k}\right)+\hat{a}_{\vec{q}+\vec{p}}^{a c c^{\prime} b^{\prime} k^{\prime} k}\left(q_{i^{\prime}}+2 p_{i^{\prime}}\right)\left(q_{k}-p_{k}\right)\right)\left\langle\widetilde{A}_{c i}^{\dagger r \vec{p}}(t) \widetilde{A}_{b^{\prime} k^{\prime}}^{r \vec{p}}\left(t^{\prime}\right)\right\rangle \\
& +\hat{a}_{\vec{q}-\vec{p}^{a} k^{\prime} k^{\prime} k}^{a c c^{\prime}}\left(q_{k^{\prime}}-p_{k^{\prime}}\right)\left(q_{k}+p_{k}\right)\left\langle\widetilde{A}_{c i}^{r \vec{p}}(t) \widetilde{A}_{b^{\prime} i^{\prime}}^{\dagger r \vec{p}}\left(t^{\prime}\right)\right\rangle \\
& +\hat{a}_{\vec{q}+\vec{p}}^{a c c^{\prime} b^{\prime} k^{\prime} k}\left(q_{k^{\prime}}+p_{k^{\prime}}\right)\left(q_{k}-p_{k}\right)\left\langle\widetilde{A}_{c i}^{\dagger r \vec{p}}(t) \widetilde{A}_{b^{\prime} i^{\prime}}^{r \vec{p}}\left(t^{\prime}\right)\right\rangle \\
& +\left(\hat{a}_{\vec{q}-\vec{p}}^{a b b^{\prime} i^{\prime} i^{\prime} k}\left(q_{k^{\prime}}-p_{k^{\prime}}\right)\left(q_{i}-2 p_{i}\right)+\hat{a}_{\vec{q}-\vec{p}}^{a b b^{\prime} k^{\prime} i}\left(q_{i^{\prime}}-p_{i^{\prime}}\right)\left(2 q_{k}-3 p_{k}\right)\right.
\end{aligned}
$$




$$
\begin{aligned}
& \left.+\hat{a}_{\vec{q}-\vec{p}}^{a b c^{\prime} k^{\prime} k}\left(q_{i^{\prime}}-2 p_{i^{\prime}}\right)\left(q_{i}-2 p_{i}\right)+\hat{a}_{\vec{q}-\vec{p}}^{a b c^{\prime} i^{\prime} i^{\prime} i}\left(2 q_{k^{\prime}}-p_{k^{\prime}}\right)\left(2 q_{k}-3 p_{k}\right)\right)\left\langle\widetilde{A}_{b k}^{r \vec{p}}(t) \widetilde{A}_{b^{\prime} k^{\prime}}^{\dagger r \vec{p}}\left(t^{\prime}\right)\right\rangle \\
& +\left(\hat{a}_{\vec{q}+\vec{p}}^{a b b^{\prime} i^{\prime} i^{\prime} k}\left(q_{k^{\prime}}+p_{k^{\prime}}\right)\left(q_{i}+2 p_{i}\right)+\hat{a}_{\vec{q}+\vec{p}}^{a b \vec{p}^{\prime} b^{\prime} k^{\prime} i}\left(q_{i^{\prime}}+p_{i^{\prime}}\right)\left(2 q_{k}+3 p_{k}\right)\right. \\
& \left.+\hat{a}_{\vec{q}+\vec{p}}^{a b c^{\prime} k^{\prime} k^{\prime} k}\left(q_{i^{\prime}}+2 p_{i^{\prime}}\right)\left(q_{i}+2 p_{i}\right)+\hat{a}_{\vec{q}+\vec{p}}^{a b b^{\prime} i^{\prime} i^{\prime} i}\left(2 q_{k^{\prime}}+p_{k^{\prime}}\right)\left(2 q_{k}+3 p_{k}\right)\right)\left\langle\widetilde{A}_{b k}^{\dagger r \vec{p}}(t) \widetilde{A}_{b^{\prime} k^{\prime}}^{r \vec{p}}\left(t^{\prime}\right)\right\rangle \\
& +\left(\hat{a}_{\vec{q}-\vec{p}}^{a b c^{\prime} \vec{b}^{\prime} k^{\prime} i}\left(q_{k^{\prime}}-p_{k^{\prime}}\right)\left(2 q_{k}-3 p_{k}\right)+\hat{a}_{\vec{q}-\vec{p}}^{a b c^{\prime} \vec{b}^{\prime} k^{\prime} k}\left(q_{k^{\prime}}-p_{k^{\prime}}\right)\left(q_{i}-2 p_{i}\right)\right)\left\langle\widetilde{A}_{b k}^{r \vec{p}}(t) \widetilde{A}_{b^{\prime} i^{\prime}}^{\dagger r \vec{p}}\left(t^{\prime}\right)\right\rangle \\
& +\left(\hat{a}_{\vec{q}+\vec{p}}^{a b c^{\prime} k^{\prime} k^{\prime} i}\left(q_{k^{\prime}}+p_{k^{\prime}}\right)\left(2 q_{k}+3 p_{k}\right)\right. \\
& \left.\left.+\hat{a}_{\vec{q}+\vec{p}}^{a b b^{\prime} k^{\prime} k}\left(q_{k^{\prime}}+p_{k^{\prime}}\right)\left(q_{i}+2 p_{i}\right)\right)\left\langle\widetilde{A}_{b k}^{\dagger r \vec{p}}(t) \widetilde{A}_{b^{\prime} i^{\prime}}^{r \vec{p}}\left(t^{\prime}\right)\right\rangle\right]
\end{aligned}
$$

Here, the Fourier coefficients are given by

$$
\begin{aligned}
\hat{a}_{\vec{p}}^{a c b^{\prime} c^{\prime} i^{\prime} k} & =\frac{1}{3} f_{a b c} f_{b b^{\prime} c^{\prime}}\left(\frac{-g^{2}}{i p} e^{i p\left(t-t^{\prime}\right)} s_{i^{\prime}}^{\alpha} s_{k}^{\alpha}+\frac{g^{2}}{i p} e^{i p\left(t^{\prime}-t\right)} s_{i^{\prime}}^{\alpha} s_{k}^{\alpha}+2 g^{2} n_{i^{\prime}} n_{k}\left(t^{\prime}-t\right)\right), \\
\hat{a}_{0}^{a c b^{\prime} c^{\prime} i^{\prime} k} & =\frac{2}{3} f_{a b c} f_{b b^{\prime} c^{\prime}} g^{2} \delta_{i^{\prime} k}\left(t^{\prime}-t\right) .
\end{aligned}
$$

Equation (5.29) describes dynamics of the gluon plasma including the contribution of all the terms terms up to $g^{2}$ in eq. (5.1). One observes that the second-order self-interactions induce a time non-local contribution to the effective gluon mass compared to the local ones induced by the third-order self-interactions.

\subsection{Equations for condensate and waves with all wave self-interaction terms up to $g^{2}$ order}

All the relevant interaction terms up to $g^{2}$ order can be incorporated into the equations of motion for the YM "condensate + waves" system in an analogical way as was done above in section 5.2 for the pure gluon plasma case. Before separation of the YM condensate from YM fields, the YM equations of motion can be written in terms of "real" and "virtual" fields in the form of eqs. (5.24) and (5.25) where wave modes should replaced by complete fields everywhere, i.e. $\widetilde{A} \rightarrow A$. Correspondingly, the solutions for $A^{v}$ in terms of $A^{r}$ have exactly the same form as eqs. (5.26)-(5.28) but with replacements $\widetilde{A} \rightarrow A$. Using the latter expressions in the equation for the "real" part one completely excludes the "virtual" components from consideration and analyzes dynamics of the largest "real" part only.

Let us now consider for simplicity SU(2) theory and separate of the YM condensate and wave components in the "real" part in usual manner

$$
A_{i k}^{r}(t, \vec{x})=\delta_{i k} U(t)+\widetilde{A}_{i k}^{r}(t, \vec{x}) .
$$

Then one ends up with an integro-differential equation for the corresponding condensate

$$
\begin{aligned}
& \partial_{0} \partial_{0} U+2 g^{2} U^{3}+\frac{1}{3} \int_{t_{0}}^{t} d t^{\prime} U\left(t^{\prime}\right) M_{a a b b}^{0}\left(t, t^{\prime}\right)+\frac{g^{2}}{36} U \sum_{\vec{p}}\left\langle\widetilde{A}_{c k}^{r \vec{p} \dagger} \widetilde{A}_{c k}^{r \vec{p}}+\widetilde{A}_{c k}^{r \vec{p}} \widetilde{A}_{c k}^{r \vec{p} \dagger}\right. \\
&\left.+2 \widetilde{A}_{a a}^{r \vec{p} \dagger} \widetilde{A}_{i i}^{r \vec{p}}+2 \widetilde{A}_{a a}^{r \vec{p}} \widetilde{A}_{i i}^{r \vec{p} \dagger}-\widetilde{A}_{c k}^{r \vec{p} \dagger} \widetilde{A}_{k c}^{r \vec{p}}-\widetilde{A}_{c k}^{r \vec{p}} \widetilde{A}_{k c}^{r \vec{p} \dagger}\right\rangle=0,
\end{aligned}
$$

where $M_{a a b b}^{0}\left(t, t^{\prime}\right) \equiv M_{a a b b}^{\vec{q}=0}\left(t, t^{\prime}\right)$ matrix should be found from eq. (5.30) for $\vec{q}=0$. 
The equation for the YM waves takes the form

$$
\begin{aligned}
& \partial_{0} \partial_{0} \widetilde{A}_{a i}^{r \vec{q}}+q^{2} \widetilde{A}_{a i}^{r \vec{q}}-q_{i} q_{k} \widetilde{A}_{a k}^{r \vec{q}}+\int_{t 0}^{t} \widetilde{A}_{c^{\prime} i^{\prime}}^{r \vec{q}}\left(t^{\prime}\right) M_{a i c^{\prime} i^{\prime}}^{\vec{q}}\left(t, t^{\prime}\right) d t^{\prime} \\
& +\widetilde{A}_{c l}^{r \vec{q}}(t) \int_{t_{0}}^{t} d t^{\prime} \sum_{\vec{p}} N_{a i c l}^{\vec{p} \vec{q}}\left(t, t^{\prime}\right)+U(t) \int_{t_{0}}^{t} d t^{\prime} U\left(t^{\prime}\right) P_{a i b^{\prime} k^{\prime}}^{\vec{q}} \widetilde{A}_{b^{\prime} k^{\prime}}^{r \vec{q}}\left(t^{\prime}\right) \\
& +\frac{g^{2}}{3} U^{2}\left(\widetilde{A}_{a i}^{r \vec{q}}-\widetilde{A}_{i a}^{r \vec{q}}+2 \delta_{a i} \widetilde{A}_{k k}^{r \vec{q}}\right)+g^{2}\left(2 \widetilde{A}_{b k}^{r \vec{q}} m_{a i}^{b k}\right. \\
& \left.+\widetilde{A}_{a i}^{r \vec{q}} m_{b k}^{b k}-\widetilde{A}_{b k}^{r \vec{q}} m_{b i}^{a k}-\widetilde{A}_{a k}^{r \vec{q}} m_{b i}^{b k}-\widetilde{A}_{b i}^{r \vec{q}} m_{a k}^{b k}\right)=0
\end{aligned}
$$

where tensors $N_{a i c l}^{\vec{p} \vec{q}}\left(t, t^{\prime}\right)$ and $P_{a i b^{\prime} k^{\prime}}^{\vec{q}}$ (with coefficients $\hat{a}_{\vec{q}}, \hat{a}_{0}$ given by eq. (5.31), (5.32), respectively) are defined as follows

$$
\begin{aligned}
N_{a i c l}^{\vec{p} q}\left(t, t^{\prime}\right)= & \frac{1}{8}\left(2 q_{i} \hat{a}_{0}^{a c b^{\prime} c^{\prime} i^{\prime} l}-2 \delta_{l i} q_{k} \hat{a}_{0}^{a c b^{\prime} c^{\prime} i^{\prime} k}+q_{l} \hat{a}_{0}^{a c b^{\prime} c^{\prime} i^{\prime} i}\right)\left(2 p_{k^{\prime}}\left\langle\widetilde{A}_{c^{\prime} i^{\prime}}^{r \vec{p}} \widetilde{A}_{b^{\prime} k^{\prime}}^{r \vec{p} \dagger}\right\rangle\right. \\
& -2 p_{k^{\prime}}\left\langle\widetilde{A}_{c^{\prime} i^{\prime}}^{r \overrightarrow{p^{\prime}}} \widetilde{A}_{b^{\prime} k^{\prime}}^{r \vec{p}}\right\rangle+p_{i^{\prime}}\left\langle\widetilde{A}_{b^{\prime} k^{\prime}}^{r \vec{p}} \widetilde{A}_{c^{\prime} k^{\prime}}^{r \vec{p} \dagger}\right\rangle \\
& \left.-p_{i^{\prime}}\left\langle\widetilde{A}_{b^{\prime} k^{\prime}}^{r \vec{p} \dagger} \widetilde{A}_{c^{\prime} k^{\prime}}^{r \vec{p}}\right\rangle+p_{k^{\prime}}\left\langle\widetilde{A}_{b^{\prime} k^{\prime}}^{r \vec{p}} \widetilde{A}_{c^{\prime} i^{\prime}}^{r \vec{p} \dagger}\right\rangle-p_{k^{\prime}}\left\langle\widetilde{A}_{b^{\prime} k^{\prime}}^{r \vec{p} \dagger} \widetilde{A}_{c^{\prime} i^{\prime}}^{r \vec{p}}\right\rangle\right), \\
P_{a i b^{\prime} k^{\prime}}^{\vec{q}}\left(t, t^{\prime}\right)= & 2 q_{l} \delta_{k^{\prime} i}\left(q_{k} \hat{a}_{\vec{q}}^{a k l b^{\prime} i^{\prime} i}-\frac{1}{2} q_{i} \hat{a}_{\vec{q}}^{a k l b^{\prime} i^{\prime} k}-\frac{1}{2} q_{k} \hat{a}_{\vec{q}}^{a i l b^{\prime} i^{\prime} k}\right) \\
& +q_{i^{\prime}}\left(q_{k} \hat{a}_{\vec{q}}^{a k b^{\prime} k^{\prime} i^{\prime} i}-\frac{1}{2} q_{i} \hat{a}_{\vec{q}}^{a k b^{\prime} k^{\prime} i^{\prime} k}-\frac{1}{2} q_{k} \hat{a}_{\vec{q}}^{a i b^{\prime} k^{\prime} i^{\prime} k}\right) \\
& +q_{k^{\prime}}\left(q_{k} \hat{a}_{\vec{q}}^{a k b^{\prime} i^{\prime} i^{\prime} i}-\frac{1}{2} q_{i} \hat{a}_{\vec{q}}^{a k b^{\prime} i^{\prime} i^{\prime} k}-\frac{1}{2} q_{k} \hat{a}_{\vec{q}}^{a i b^{\prime} i^{\prime} i^{\prime} k}\right) .
\end{aligned}
$$

\section{Condensate and waves in a supersymmetric Yang-Mills theory}

Now we would like to extend our analysis to the $\mathcal{N}=4$ supersymmetric Yang-Mills theory with $\mathrm{SU}(2)$ which contains additional spinor, scalar and pseudo-scalar d.o.f. (see e.g. ref. [25]). The corresponding Lagrangian reads

$$
\begin{aligned}
\mathcal{L}_{\mathrm{SUSY}}= & -\frac{1}{4} F_{\mu \nu}^{a} F_{a}^{\mu \nu}+\frac{i}{2} \bar{\lambda}_{k^{\prime}}^{a} \gamma^{\mu} D_{\mu} \lambda_{k^{\prime}}^{a}+\frac{1}{2}\left(D_{\mu} C_{(i)}^{a}\right)^{2}+\frac{1}{2}\left(D_{\mu} B_{(i)}^{a}\right)^{2} \\
& -\frac{g}{2} \epsilon^{a b c} \bar{\lambda}_{k^{\prime}}^{a}\left(\alpha_{k^{\prime} l^{\prime}}^{(i)} C_{(i)}^{b}+\beta_{k^{\prime} l^{\prime}}^{(i)} \gamma_{5} B_{(i)}^{b}\right) \lambda_{l^{\prime}}^{c} \\
& +\frac{g^{2}}{4}\left[\left(\epsilon^{a b c} C_{(i)}^{b} C_{(j)}^{c}\right)^{2}+\left(\epsilon^{a b c} B_{(i)}^{b} B_{(j)}^{c}\right)^{2}+\left(\epsilon^{a b c} C_{(i)}^{b} B_{(j)}^{c}\right)^{2}\right] .
\end{aligned}
$$

Here $a, b, c=1 \ldots 3$ are the isotopic $\mathrm{SU}(2)$ indices, $i, j=1 \ldots 3$ numerate different types of scalar $C$ and pseudoscalar $B$ fields, $k^{\prime}, l^{\prime}=1 \ldots 4$ numerate different flavors of fermions $\lambda$. Next, we decompose additional supersymmetric modes into transverse and longitudinal components in momentum space as follows

$$
\begin{aligned}
C_{k}^{(i) \vec{p}} & =n_{k} K_{(i)}+s_{k}^{\alpha} M_{(i) \alpha}, \\
B_{k}^{(i) \vec{p}} & =n_{k} K_{(i)}^{\prime}+s_{k}^{\alpha} M_{(i) \alpha}^{\prime}, \\
\lambda_{k}^{k^{\prime} \vec{p}} & =n_{k} \vartheta^{k^{\prime}}+s_{k}^{\alpha} \zeta_{\alpha}^{k^{\prime}} .
\end{aligned}
$$


Also, we perform the corresponding tensor decompositions for the YM field $A_{\mu}^{a}$ which enters the covariant derivative $D_{\mu}$ and stress tensor $F_{\mu \nu}$ in the same way as is done above.

Next, let us rewrite the supersymmetric part of the Lagrangian density (6.1) in terms of new Fourier modes $K, M, \vartheta$ and $\zeta$. The $\mathcal{N}=4$ supersymmetric YM Lagrangian density (4.20) is given in terms of wave modes and condensate by

$$
\begin{aligned}
& \mathcal{L}_{\mathrm{SUSY}}=\frac{1}{2}\left\{\partial_{0} \psi_{\lambda} \partial_{0} \psi_{\lambda}^{\dagger}+\partial_{0} \phi_{\sigma} \partial_{0} \phi_{\sigma}^{\dagger}+\partial_{0} \Phi \partial_{0} \Phi^{\dagger}+\frac{1}{2} \partial_{0} \Lambda \partial_{0} \Lambda^{\dagger}+\partial_{0} \eta_{\sigma} \partial_{0} \eta_{\sigma}^{\dagger}\right. \\
&+ \partial_{0} \lambda \partial_{0} \lambda^{\dagger}-p^{2} \psi_{\lambda} \psi_{\lambda}^{\dagger}-\frac{p^{2}}{2} \phi_{\sigma} \phi_{\sigma}^{\dagger}-p^{2} \Phi \Phi^{\dagger}-\frac{p^{2}}{2} \eta_{\sigma} \eta_{\sigma}^{\dagger}-p^{2} \lambda \lambda^{\dagger} \\
&+\frac{p^{2}}{2} e^{\gamma \sigma}\left(\eta_{\sigma} \phi_{\gamma}^{\dagger}+\phi_{\gamma} \eta_{\sigma}^{\dagger}\right)-i g p U e^{\sigma \gamma} \eta_{\sigma} \eta_{\gamma}^{\dagger}+i g p U Q^{\lambda \gamma} \psi_{\lambda} \psi_{\gamma}^{\dagger} \\
&+i g p U e^{\sigma \gamma} \phi_{\sigma} \phi_{\gamma}^{\dagger}+i g p U\left(2 \Phi \lambda^{\dagger}-2 \lambda \Phi^{\dagger}+\Lambda \lambda^{\dagger}-\lambda \Lambda^{\dagger}\right) \\
&-2 g^{2} U^{2} \eta_{\sigma} \eta_{\sigma}^{\dagger}-2 g^{2} U^{2} \lambda \lambda^{\dagger}-g^{2} U^{2}\left(4 \Phi \Phi^{\dagger}+2 \Phi \Lambda^{\dagger}+2 \Lambda \Phi^{\dagger}+\Lambda \Lambda^{\dagger}\right) \\
&+\frac{1}{2} \partial_{0} K_{(i)} \partial_{0} K_{(i)}^{\dagger}+\frac{1}{2} \partial_{0} M_{(i) \alpha} \partial_{0} M_{(i) \alpha}^{\dagger}-\frac{1}{2} p^{2} K_{(i)} K_{(i)}^{\dagger}-\frac{1}{2} p^{2} M_{(i) \alpha} M_{(i) \alpha}^{\dagger} \\
&-i g p U e^{\beta \alpha} M_{(i) \alpha} M_{(i) \beta}^{\dagger}-g^{2} U^{2} K_{(i)} K_{(i)}^{\dagger} g^{2} U^{2} M_{(i) \alpha} M_{(i) \alpha}^{\dagger} \\
&+\frac{1}{2} \partial_{0} K_{(i)}^{\prime} \partial_{0} K_{(i)}^{\prime \dagger}+\frac{1}{2} \partial_{0} M_{(i) \alpha}^{\prime} \partial_{0} M_{(i) \alpha}^{\prime \dagger}-\frac{1}{2} p^{2} K_{(i)}^{\prime} K_{(i)}^{\prime \dagger}-\frac{1}{2} p^{2} M_{(i) \alpha}^{\prime} M_{(i) \alpha}^{\prime \dagger} \\
&-i g p U e^{\beta \alpha} M_{(i) \alpha}^{\prime} M_{(i) \beta}^{\prime \dagger}-g^{2} U^{2} K_{(i)}^{\prime} K_{(i)}^{\prime \dagger} g^{2} U^{2} M_{(i) \alpha}^{\prime} M_{(i) \alpha}^{\prime \dagger} \\
&+\frac{i}{2} \bar{\vartheta}^{k^{\prime}} \gamma_{0} \partial_{0} \vartheta^{k^{\prime}}+\frac{i}{2} \bar{\zeta}_{\alpha}^{k^{\prime}} \gamma_{0} \partial_{0} \zeta_{\alpha}^{k^{\prime}}-\frac{p}{2} n_{k} \bar{\vartheta}^{k^{\prime}} \gamma_{k} \vartheta^{k^{\prime}}-\frac{p}{2} n_{k} \bar{\zeta}_{\alpha}^{k^{\prime}} \gamma_{k} \zeta_{\alpha}^{k^{\prime}} \\
&\left.-\frac{i}{2} g U e_{i l m} n_{i} s_{m}^{\alpha} \bar{\vartheta}^{k^{\prime}} \gamma_{l} \zeta_{\alpha}^{k^{\prime}}-\frac{i}{2} g U e_{i l m} n_{m} s_{i}^{\alpha} \bar{\zeta}_{\alpha}^{k^{\prime}} \gamma_{l} \vartheta^{k^{\prime}}-\frac{i}{2} g U e_{i l m} s_{i}^{\alpha} s_{m}^{\beta} \bar{\zeta}_{\alpha}^{k^{\prime}} \gamma_{l} \zeta_{\beta}^{k^{\prime}}\right\}
\end{aligned}
$$

and the corresponding Hamiltonian density (4.21) has a form

$$
\begin{aligned}
& \mathcal{H}_{\mathrm{SUSY}}=\frac{1}{2}\left\{\partial_{0} \psi_{\lambda} \partial_{0} \psi_{\lambda}^{\dagger}+\partial_{0} \phi_{\sigma} \partial_{0} \phi_{\sigma}^{\dagger}+\partial_{0} \Phi \partial_{0} \Phi^{\dagger}+\frac{1}{2} \partial_{0} \Lambda \partial_{0} \Lambda^{\dagger}+\partial_{0} \eta_{\sigma} \partial_{0} \eta_{\sigma}^{\dagger}\right. \\
&+\partial_{0} \lambda \partial_{0} \lambda^{\dagger}+p^{2} \psi_{\lambda} \psi_{\lambda}^{\dagger}+\frac{p^{2}}{2} \phi_{\sigma} \phi_{\sigma}^{\dagger}+p^{2} \Phi \Phi^{\dagger}+\frac{p^{2}}{2} \eta_{\sigma} \eta_{\sigma}^{\dagger}+p^{2} \lambda \lambda^{\dagger} \\
&+\frac{p^{2}}{2} e^{\gamma \sigma}\left(\eta_{\sigma} \phi_{\gamma}^{\dagger}+\phi_{\gamma} \eta_{\sigma}^{\dagger}\right)+i g p U e^{\sigma \gamma} \eta_{\sigma} \eta_{\gamma}^{\dagger}-i g p U Q^{\lambda \gamma} \psi_{\lambda} \psi_{\gamma}^{\dagger} \\
&+i g p U e^{\sigma \gamma} \phi_{\sigma} \phi_{\gamma}^{\dagger}-i g p U\left(2 \Phi \lambda^{\dagger}-2 \lambda \Phi^{\dagger}+\Lambda \lambda^{\dagger}-\lambda \Lambda^{\dagger}\right) \\
&+ 2 g^{2} U^{2} \eta_{\sigma} \eta_{\sigma}^{\dagger}+2 g^{2} U^{2} \lambda \lambda^{\dagger}+g^{2} U^{2}\left(4 \Phi \Phi^{\dagger}+2 \Phi \Lambda^{\dagger}+2 \Lambda \Phi^{\dagger}+\Lambda \Lambda^{\dagger}\right) \\
&+\frac{1}{2} \partial_{0} K_{(i)} \partial_{0} K_{(i)}^{\dagger}+\frac{1}{2} \partial_{0} M_{(i) \alpha} \partial_{0} M_{(i) \alpha}^{\dagger}+\frac{1}{2} p^{2} K_{(i)} K_{(i)}^{\dagger}+\frac{1}{2} p^{2} M_{(i) \alpha} M_{(i) \alpha}^{\dagger} \\
&+i g p U e^{\beta \alpha} M_{(i) \alpha} M_{(i) \beta}^{\dagger}+g^{2} U^{2} K_{(i)} K_{(i)}^{\dagger}+g^{2} U^{2} M_{(i) \alpha} M_{(i) \alpha}^{\dagger} \\
&+\frac{1}{2} \partial_{0} K_{(i)}^{\prime} \partial_{0} K_{(i)}^{\prime \dagger}+\frac{1}{2} \partial_{0} M_{(i) \alpha}^{\prime} \partial_{0} M_{(i) \alpha}^{\prime \dagger}+\frac{1}{2} p^{2} K_{(i)}^{\prime} K_{(i)}^{\prime \dagger}+\frac{1}{2} p^{2} M_{(i) \alpha}^{\prime} M_{(i) \alpha}^{\prime \dagger}
\end{aligned}
$$




$$
\begin{aligned}
& +i g p U e^{\beta \alpha} M_{(i) \alpha}^{\prime} M_{(i) \beta}^{\prime \dagger}+g^{2} U^{2} K_{(i)}^{\prime} K_{(i)}^{\prime \dagger} g^{2} U^{2} M_{(i) \alpha}^{\prime} M_{(i) \alpha}^{\prime \dagger} \\
& +\frac{p}{2} n_{k} \bar{\vartheta}^{k^{\prime}} \gamma_{k} \vartheta^{k^{\prime}}+\frac{p}{2} n_{k} \bar{\zeta}_{\alpha}^{k^{\prime}} \gamma_{k} \zeta_{\alpha}^{k^{\prime}}+\frac{i}{2} g U e_{i l m} n_{i} s_{m}^{\alpha} \bar{\vartheta}^{k^{\prime}} \gamma_{l} \zeta_{\alpha}^{k^{\prime}} \\
& \left.+\frac{i}{2} g U e_{i l m} n_{m} s_{i}^{\alpha} \bar{\zeta}_{\alpha}^{k^{\prime}} \gamma_{l} \vartheta^{k^{\prime}}+\frac{i}{2} g U e_{i l m} s_{i}^{\alpha} s_{m}^{\beta} \bar{\zeta}_{\alpha}^{k^{\prime}} \gamma_{l} \zeta_{\beta}^{k^{\prime}}\right\}
\end{aligned}
$$

The equations of motion for the extra supersymmetric d.o.f. $K, M, \vartheta^{k^{\prime}}, \zeta_{\alpha}^{k^{\prime}}$ can be constructed in the standard way as Lagrange (or Hamilton) equations based upon eq. (6.2) (or eq. (6.3))

$$
\begin{aligned}
\partial_{0} \partial_{0} K_{(i)}+p^{2} K_{(i)}+2 g^{2} U^{2} K_{(i)} & =0, \\
\partial_{0} \partial_{0} M_{(i) \alpha}+p^{2} M_{(i) \alpha}+2 i g p U e^{\alpha \beta} M_{\beta}+2 g^{2} U^{2} M_{(i) \alpha} & =0, \\
\gamma_{0} \partial_{0} \vartheta^{k^{\prime}}+i p n_{j} \gamma_{j} \vartheta^{k^{\prime}}-g U e_{i k l} \gamma_{k} n_{i} s_{l}^{\alpha} \zeta_{\alpha}^{k^{\prime}} & =0, \\
\gamma_{0} \partial_{0} \zeta_{\alpha}^{k^{\prime}}+i p n_{j} \gamma_{j} \zeta_{\alpha}^{k^{\prime}}-g U e_{i k l} \gamma_{k} s_{i}^{\alpha} s_{l}^{\beta} \zeta_{\beta}^{k^{\prime}}-g U e_{i k l} \gamma_{k} n_{l} s_{i}^{\alpha} \vartheta^{k^{\prime}} & =0 .
\end{aligned}
$$

The equations for pseudoscalar modes $K^{\prime}$ and $M^{\prime}$ are the same as equations for $K(6.4)$ and $M$ (6.5), respectively. A numerical analysis of these equations in the linear approximation (with free YM condensate) has shown that qualitative behavior of the (pseudo)scalar modes is analogical to that of the YM wave modes discussed above. Finally, the equation of motion for the YM condensate in the next-to-linear approximation accounting for "back reaction" effects of the wave modes (including supersymmetric ones) to the condensate reads

$$
\begin{aligned}
& \partial_{0} \partial_{0} U+2 g^{2} U^{3}+\frac{g^{2}}{6} U \sum_{\vec{p}}\left\langle 2 \eta_{\sigma} \eta_{\sigma}^{\dagger}+2 \lambda \lambda^{\dagger}+4 \Phi \Phi^{\dagger}+\Lambda \Lambda^{\dagger}+2 \Phi \Lambda^{\dagger}\right. \\
& \left.+2 \Lambda \Phi^{\dagger}+2 \eta_{\sigma}^{\dagger} \eta_{\sigma}+2 \lambda^{\dagger} \lambda+4 \Phi^{\dagger} \Phi+\Lambda^{\dagger} \Lambda+2 \Phi^{\dagger} \Lambda+2 \Lambda^{\dagger} \Phi\right\rangle \\
& +\frac{i g}{12} \sum_{\vec{p}} p\left\langle 2 \Lambda^{\dagger} \lambda-2 \Lambda \lambda^{\dagger}+2 \Phi^{\dagger} \lambda-2 \Phi \lambda^{\dagger}+2 \lambda \Phi^{\dagger}-2 \lambda^{\dagger} \Phi\right. \\
& -Q^{\lambda \sigma}\left(\psi_{\lambda} \psi_{\sigma}^{\dagger}-\psi_{\lambda}^{\dagger} \psi_{\sigma}\right)-e^{\sigma \gamma}\left(\phi_{\sigma} \phi_{\gamma}^{\dagger}-\phi_{\sigma}^{\dagger} \phi_{\gamma}\right) \\
& \left.+\phi_{\sigma}^{\dagger} \eta_{\sigma}-\phi_{\sigma} \eta_{\sigma}^{\dagger}+\eta_{\sigma}^{\dagger} \phi_{\sigma}-\eta_{\sigma} \phi_{\sigma}^{\dagger}+e^{\sigma \gamma}\left(\eta_{\sigma} \eta_{\gamma}^{\dagger}-\eta_{\sigma}^{\dagger} \eta_{\gamma}\right)\right\rangle \\
& +\frac{g^{2}}{6} U \sum_{\vec{p}}\left\langle K_{(i)} K_{(i)}^{\dagger}+K_{(i)}^{\dagger} K_{(i)}+M_{(i) \alpha}^{\dagger} M_{(i) \alpha}+M_{(i) \alpha} M_{(i) \alpha}^{\dagger}\right\rangle \\
& +\frac{i g}{12} \sum_{\vec{p}} p\left\langle e^{\alpha \beta}\left(M_{(i) \alpha}^{\dagger} M_{(i) \beta}-M_{(i) \alpha} M_{(i) \beta}^{\dagger}\right)\right\rangle \\
& +\frac{g^{2}}{6} U \sum_{\vec{p}}\left\langle K_{(i)}^{\prime} K_{(i)}^{\prime \dagger}+K_{(i)}^{\prime \dagger} K_{(i)}^{\prime}+M_{(i) \alpha}^{\prime \dagger} M_{(i) \alpha}^{\prime}+M_{(i) \alpha}^{\prime} M_{(i) \alpha}^{\prime \dagger}\right\rangle \\
& +\frac{i g}{12} \sum_{\vec{p}} p\left\langle e^{\alpha \beta}\left(M_{(i) \alpha}^{\prime \dagger} M_{(i) \beta}^{\prime}-M_{(i) \alpha}^{\prime} M_{(i) \beta}^{\prime \dagger}\right)\right\rangle \\
& -\frac{i g}{6} \sum_{\vec{p}}\left\langle e_{l m p} n_{m} s_{p}^{\alpha} \overline{\bar{\vartheta}^{k^{\prime}}} \gamma_{l} \zeta_{\alpha}^{k^{\prime}}+e_{l m p} n_{p} s_{m}^{\alpha}{\overline{\zeta_{\alpha}}}^{k^{\prime}} \gamma_{l} \vartheta^{k^{\prime}}+e_{l m p} s_{m}^{\alpha} s_{p}^{\beta} \bar{\zeta}_{\alpha}^{k^{\prime}} \gamma_{l} \zeta_{\beta}^{k^{\prime}}\right\rangle=0 .
\end{aligned}
$$


Taking into consideration only additional scalar and pseudoscalar fields in numerical analysis we notice that the qualitative picture of YM condensate dynamics shown in figures 3 is not changed. Also, energy of the extra d.o.f.'s grows effectively due to the energy swap effect in the parametric resonance-like instability region similarly to the other YM wave modes.

As a validation test of our quasi-classical approach the canonical quantization of the YM wave modes in the classical YM condensate has been performed in appendix B where the quantum Hamilton equations in commutators have been constructed and are shown to be consistent with the YM equations of motion (4.13), (4.14) and (6.4)-(6.7). Note, a consistent analysis of equations (6.6) and (6.7) for the spinor modes $\vartheta^{k^{\prime}}, \zeta_{\alpha}^{k^{\prime}}$ can only be performed in the framework of quantum field theory approach, which is planned for further studies.

\section{Cosmological evolution of the Yang-Mills condensate}

Until now, we have discussed the properties of the YM condensates in trivial Minkowski background. It is instructive to extend this consideration to a realistic case of flat Friedmann universe since these findings can be useful e.g. in studies of the cosmological QCD phase transition, QCD vacuum compensation to the Dark Energy, particle production mechanisms after Inflation etc.

\subsection{Free condensate case}

To start with, let us neglect the wave modes and study evolution of the homogeneous YM condensate $U=U(t)$ in the $\mathrm{SU}(2)$ theory in the early Universe disregarding all other forms of matter. The 4-interval in the flat Friedmann model is

$$
d s^{2}=a(\eta)^{2}\left(d \eta^{2}-d x^{2}-d y^{2}-d z^{2}\right),
$$

where $\eta$ is the conformal time related to the cosmological time as

$$
d t=a d \eta
$$

The corresponding metric is

$$
g_{\mu \nu}=a^{2} g_{\mu \nu}^{M},
$$

where $g_{\mu \nu}^{M}$ is the Minkowski metric. The corresponding Einstein equations with conformal metric (7.3) and energy-momentum tensor of the classical Yang-Mills fields read [34-36]

$$
\begin{aligned}
& \frac{1}{\varkappa}\left(R_{\mu}^{\nu}-\frac{1}{2} \delta_{\mu}^{\nu} R\right)=\frac{1}{g_{\mathrm{YM}}^{2}} \frac{1}{\sqrt{-g}}\left(-F_{\mu \lambda}^{a} F_{a}^{\nu \lambda}+\frac{1}{4} \delta_{\mu}^{\nu} F_{\sigma \lambda}^{a} F_{a}^{\sigma \lambda}\right), \quad \sqrt{-g}=a^{4}(\eta), \\
& \left(\frac{\delta^{a b}}{\sqrt{-g}} \partial_{\nu} \sqrt{-g}-f^{a b c} A_{\nu}^{c}\right) \frac{F_{b}^{\mu \nu}}{\sqrt{-g}}=0, \quad F_{\mu \nu}^{a}=\partial_{\mu} A_{\nu}^{a}-\partial_{\nu} A_{\mu}^{a}+f^{a b c} A_{\mu}^{b} A_{\nu}^{c},
\end{aligned}
$$

where the gluon vacuum polarisation effects are not included in the considered free YM condensate case. As usual, raising and lowering the Lorenz indices are done by the Minkowski metric $g_{\mu \nu(\mathrm{M})}$. 
Neglecting the wave modes, the classical Einstein-Yang-Mills equations (7.4) for the condensate $U=U(\eta)$ and the scale factor $a=a(\eta)$ are reduced to

$$
\frac{3}{\varkappa} \frac{a^{\prime 2}}{a^{4}}=\frac{3}{2 g_{\mathrm{YM}}^{2} a^{4}}\left(U^{\prime 2}+U^{4}\right), \quad U^{\prime \prime}+2 U^{3}=0,
$$

The exact general solution of this system for the conformal evolution of the YM condensate corresponds to the non-linear oscillation whose frequency depends on initial value of the condensate $U_{0}=U(0)$, namely

$$
\begin{array}{rlrl}
U^{\prime 2}+U^{4} & =C^{4}, & \int_{U_{0}}^{U} \frac{d U}{\sqrt{C^{4}-U^{4}}} & =\eta, \\
U(\eta) & \simeq U_{0} \cos \left(\frac{6}{5} U_{0} \eta\right), & C & =U_{0}, \\
U^{\prime}(0)=0,
\end{array}
$$

where $U_{0}, C$ are the integration constants. We notice that the YM condensate in the Friedmann Universe behaves as an ultra-relativistic medium with energy density $\varepsilon_{\mathrm{YM}} \sim$ $1 / a^{4}$ and the equation of state $p_{\mathrm{YM}}=\varepsilon_{\mathrm{YM}} / 3[37]$.

\subsection{Quasi-free condensate and vacuum polarisation effects}

Let us discuss the conformal time evolution of the quasi-free YM condensate $U=U(\eta)$ in the SU(2) theory in the Friedmann Universe incorporating the vacuum polarisation effects. The corresponding YM Lagrangian accounting the one-loop vacuum polarisation reads [38]:

$$
L_{\mathrm{YM}}=-\frac{11}{128 \pi^{2}} \frac{F_{\mu \nu}^{a} F_{a}^{\mu \nu}}{\sqrt{-g}} \ln \left(\frac{J}{\Lambda_{\mathrm{QCD}}^{4}}\right), \quad J=\frac{1}{\xi^{4}} \frac{\left|F_{\alpha \beta}^{a} F_{a}^{\alpha \beta}\right|}{\sqrt{-g}},
$$

where parameter $\xi$ reflects an ambiguity in normalisation of the invariant $J$. The corresponding Einstein-Yang-Mills equations now read [17]

$$
\begin{aligned}
& \frac{1}{\varkappa}\left(R_{\mu}^{\nu}-\frac{1}{2} \delta_{\mu}^{\nu} R\right)= T_{\mu}^{\nu, \text { mat }}+\bar{\Lambda} \delta_{\mu}^{\nu}+\frac{11}{32 \pi^{2}} \frac{1}{\sqrt{-g}}\left[\left(-F_{\mu \lambda}^{a} F_{a}^{\nu \lambda}\right.\right. \\
&\left.\left.+\frac{1}{4} \delta_{\mu}^{\nu} F_{\sigma \lambda}^{a} F_{a}^{\sigma \lambda}\right) \ln \frac{e\left|F_{\alpha \beta}^{a} F_{a}^{\alpha \beta}\right|}{\sqrt{-g}\left(\xi \Lambda_{\mathrm{QCD}}\right)^{4}}-\frac{1}{4} \delta_{\mu}^{\nu} F_{\sigma \lambda}^{a} F_{a}^{\sigma \lambda}\right], \\
&\left(\frac{\delta^{a b}}{\sqrt{-g}} \partial_{\nu} \sqrt{-g}-f^{a b c} A_{\nu}^{c}\right)\left(\frac{F_{b}^{\mu \nu}}{\sqrt{-g}} \ln \frac{e\left|F_{\alpha \beta}^{a} F_{a}^{\alpha \beta}\right|}{\sqrt{-g}\left(\xi \Lambda_{\mathrm{QCD}}\right)^{4}}\right)=0,
\end{aligned}
$$

where $e \simeq 2.71$ is the base of the natural logarithm; $\Lambda_{\mathrm{QCD}}$ is the QCD energy scale; $T_{\mu}^{\nu, \text { mat }}=(\varepsilon+p) u_{\mu} u^{\nu}-\delta_{\mu}^{\nu} p$ is the energy-momentum tensor of usual matter and radiation components; $\bar{\Lambda}$ is the constant vacuum energy density.

In the considered limit of vanishing YM waves, the components of the total energymomentum tensor read

$$
\begin{aligned}
& T_{0}^{0, \text { tot }}=T_{0}^{0, \text { mat }}+\bar{\Lambda}+\frac{33}{64 \pi^{2}} \frac{1}{a^{4}}\left[\left(U^{\prime 2}+U^{4}\right) \ln \frac{6 e\left|U^{\prime 2}-U^{4}\right|}{a^{4}\left(\xi \Lambda_{\mathrm{QCD}}\right)^{4}}+U^{\prime 2}-U^{4}\right] \\
& T_{0}^{\beta, \text { tot }}=T_{0}^{\beta, \text { mat }} \\
& T_{\alpha}^{\beta, \text { tot }}=T_{\alpha}^{\beta, \text { mat }}+\bar{\Lambda} \delta_{\alpha}^{\beta}+\frac{11}{32 \pi^{2}} \frac{1}{a^{4}} \delta_{\alpha}^{\beta}\left[-\frac{1}{2}\left(U^{\prime 2}+U^{4}\right) \ln \frac{6 e\left|U^{\prime 2}-U^{4}\right|}{a^{4}\left(\xi \Lambda_{\mathrm{QCD}}\right)^{4}}+\frac{3}{2}\left(U^{\prime 2}-U^{4}\right)\right] .
\end{aligned}
$$


The trace of the Einstein equations and the equation of motion of the YM condensate are written as follows

$$
\begin{aligned}
& \frac{6}{\varkappa} \frac{a^{\prime \prime}}{a^{3}}=\varepsilon-3 p+4 \bar{\Lambda}+T_{\mu}^{\mu, \mathrm{YM}}, \quad T_{\mu}^{\mu, \mathrm{YM}}=\frac{33}{16 \pi^{2}} \frac{1}{a^{4}}\left(U^{\prime 2}-U^{4}\right), \\
& \frac{\partial}{\partial \eta}\left(U^{\prime} \ln \frac{6 e\left|U^{\prime 2}-U^{4}\right|}{a^{4}\left(\xi \Lambda_{\mathrm{QCD}}\right)^{4}}\right)+2 U^{3} \ln \frac{6 e\left|U^{\prime 2}-U^{4}\right|}{a^{4}\left(\xi \Lambda_{\mathrm{QCD}}\right)^{4}}=0,
\end{aligned}
$$

respectively. It turns out that the $(0,0)$ Einstein equation

$$
\frac{3}{\varkappa} \frac{a^{\prime 2}}{a^{4}}=\varepsilon+\bar{\Lambda}+\frac{33}{64 \pi^{2}} \frac{1}{a^{4}}\left[\left(U^{\prime 2}+U^{4}\right) \ln \frac{6 e\left|U^{\prime 2}-U^{4}\right|}{a^{4}\left(\xi \Lambda_{\mathrm{QCD}}\right)^{4}}+U^{\prime 2}-U^{4}\right]
$$

is the exact first integral of the system of equations (7.11) and (7.12), while the exact first integral of second equation (7.12) is

$$
U^{\prime 2}-U^{4}=a^{4} \frac{\left(\xi \Lambda_{\mathrm{QCD}}\right)^{4}}{6 e} .
$$

Applying this relation in eq. (7.10), one arrives to

$$
\begin{aligned}
& T_{0}^{0, \text { tot }}=T_{0}^{0, \text { mat }}+\bar{\Lambda}+\frac{33}{64 \pi^{2}} \frac{\left(\xi \Lambda_{\mathrm{QCD}}\right)^{4}}{6 e} \\
& T_{\alpha}^{\beta, \text { tot }}=T_{\alpha}^{\beta, \text { mat }}+\left(\bar{\Lambda}+\frac{33}{64 \pi^{2}} \frac{\left(\xi \Lambda_{\mathrm{QCD}}\right)^{4}}{6 e}\right) \delta_{\alpha}^{\beta} .
\end{aligned}
$$

Therefore, the quasi-free YM condensate incorporating the vacuum polarisation effects already as one loop exhibits drastically different behaviour w.r.t. to the free condensate limit. Firstly, its contribution to the total energy density of the Universe appears to be constant and independent on the scale factor like it is expected for a vacuum-like medium with the equation of state $p_{\mathrm{YM}}=-\varepsilon_{\mathrm{YM}}$ compared to free condensate equation of state $p_{\mathrm{YM}}=\varepsilon_{\mathrm{YM}} / 3$. Secondly, it provides a positive contribution to the cosmological constant which would potentially allow to compensate large negative contributions to the vacuum energy density potentially coming from quantum-topological excitations contributing to $\bar{\Lambda}$, e.g. from the vacuum averaged bilinear terms in YM waves $\left\langle\widetilde{A}^{2}\right\rangle$ [17]. The latter argument is consedered to be critical for observed smallness of the $\Lambda$-term density $\Lambda_{\text {obs }}$ which should be provided by the condition

$$
\bar{\Lambda}+\frac{33}{64 \pi^{2}} \frac{\left(\xi \Lambda_{\mathrm{QCD}}\right)^{4}}{6 e} \equiv \Lambda_{\mathrm{obs}}
$$

such that the macroscopic expansion of the Universe is driven by the ordinary Friedmann equation

$$
\frac{3}{\varkappa} \frac{a^{\prime 2}}{a^{4}}=\varepsilon+\Lambda_{\mathrm{obs}} .
$$

A thorough analysis of cosmological evolution of the complete YM system "condensate + waves" incorporating vacuum polarisation effects is planned for future studies. 


\subsection{Super-Yang-Mills condensate decay in quasi-linear approximation}

Let us discuss now the effect of wave-condensate interactions in quasi-linear approximation in the expanding Universe. For this purpose, consider the Lagrangian of $\mathcal{N}=4$ supersymmetric $\mathrm{SU}(2)$ model in arbitrary metric $g_{\mu \nu}$ in the quadratic approximation in waves (in what follows, we denote determinant of spacetime metric as $g_{\text {det }}$ in order to not confuse it up with the coupling constant)

$$
\begin{gathered}
\mathcal{L} \sqrt{-g_{\mathrm{det}}}=\sqrt{-g_{\operatorname{det}}}\left(-\frac{1}{4} F_{\mu \nu}^{a} F_{\lambda \theta}^{a} g^{\lambda \mu} g^{\theta \nu}+\frac{i}{2} \bar{\lambda}_{k^{\prime}}^{a} \gamma_{\lambda} g^{\lambda \mu} D_{\mu} \lambda_{k^{\prime}}^{a}+\frac{1}{2}\left(D_{\lambda} C_{(i)}^{a}\right)\left(D_{\mu} C_{(i)}^{a}\right) g^{\lambda \mu}\right. \\
\left.+\frac{1}{2}\left(D_{\lambda} B_{(i)}^{a}\right)\left(D_{\mu} B_{(i)}^{a}\right) g^{\lambda \mu}\right) .
\end{gathered}
$$

This Lagrangian is not conformally invariant due to the presence of scalar and pseudo-scalar fields. Note, the conformal invariance can be easily restored by adding terms proportional to the scalar curvature $R$, i.e.

$$
\begin{aligned}
\mathcal{L} \sqrt{-g_{\mathrm{det}}}=\sqrt{-g_{\mathrm{det}}} & \left(-\frac{1}{4} F_{\mu \nu}^{a} F_{\lambda \theta}^{a} g^{\lambda \mu} g^{\theta \nu}+\frac{i}{2} \bar{\lambda}_{k^{\prime}}^{a} \gamma_{\lambda} g^{\lambda \mu} D_{\mu} \lambda_{k^{\prime}}^{a}+\frac{1}{2}\left(D_{\lambda} C_{(i)}^{a}\right)\left(D_{\mu} C_{(i)}^{a}\right) g^{\lambda \mu}\right. \\
& \left.+\frac{1}{2}\left(D_{\lambda} B_{(i)}^{a}\right)\left(D_{\mu} B_{(i)}^{a}\right) g^{\lambda \mu}+\frac{1}{12} R C_{(i)}^{a} C_{(i)}^{a}+\frac{1}{12} R B_{(i)}^{a} B_{(i)}^{a}\right)
\end{aligned}
$$

The corresponding theory is free of radiative corrections and hence vacuum polarisation effects considered above due to conformal symmetry. So this model can be convenient for studies of conformal time evolution of the YM condensate induced purely by its interactions with the wave modes, at least, at the first step.

It is instructive to introduce the following conventions

$$
A_{\mu}=A_{\mu}^{M}, \quad A^{\mu}=\frac{1}{a^{2}} A_{M}^{\mu}, \quad C_{(i)}^{a}=\frac{1}{a} C_{(i)}^{M a}, \quad B_{(i)}^{a}=\frac{1}{a} B_{(i)}^{M a}, \quad \lambda_{k^{\prime}}^{a}=\frac{1}{a} \lambda_{k^{\prime}}^{M a},
$$

Then performing transition to Friedman metric (7.3) we arrive at the same expression for Lagrangian as for Minkowski metric (with $a=1$ ) due to the conformal invariance of eq. (7.19). Indeed,

$$
\begin{aligned}
\mathcal{L} \sqrt{-g_{\mathrm{det}}}= & -\frac{1}{4} F_{\mu \nu}^{M a} F_{M a}^{\mu \nu}+\frac{i}{2} \bar{\lambda}_{k^{\prime}}^{M a} \gamma^{\mu} D_{\mu} \lambda_{k^{\prime}}^{M a}+\frac{1}{2}\left(D^{\mu} C_{(i)}^{M a}\right)\left(D_{\mu} C_{(i)}^{M a}\right) \\
& +\frac{1}{2}\left(D^{\mu} B_{(i)}^{M a}\right)\left(D_{\mu} B_{(i)}^{M a}\right),
\end{aligned}
$$

where time derivatives are taken over the conformal time $\eta$. So due to conformal invariance dynamics of the YM-fields in Friedman space corresponds to that in Minkowski metric, but in conformal time.

The Friedman equation allows to find a relation between conformal and cosmological times as

$$
\frac{3}{a^{4}}\left(\frac{\partial a}{\partial \eta}\right)^{2}=\frac{\kappa}{a^{4}}\left\langle T_{0}^{0^{M}}\right\rangle, \quad\left\langle T_{0}^{0^{M}}\right\rangle \equiv c=\text { const },
$$

where $T_{0}^{0^{M}}=a^{4} T_{0}^{0}$. The corresponding solution is

$$
a=\sqrt{\frac{\kappa c}{3}} \eta, \quad t=\frac{1}{2} \sqrt{\frac{\kappa c}{3}} \eta^{2} .
$$


Our analysis of quasilinear YM theory shows that the YM condensate decay time is inversely proportional to the initial amplitude of YM condensate oscillations $U_{0}$, i.e.

$$
\Delta \eta=\frac{\alpha}{g U_{0}}
$$

where the numerical coefficient $\alpha \approx 10$ for the wave modes $\Phi, \Lambda, \lambda$. Inclusion of other modes is problematic due to the limitations of quasi-linear theory (see above) but can be consistently performed by accounting for higher order effects in waves.

Staying within the quasi-linear approximation and assuming that all the energy was concentrated in the YM condensate in the initial moment of time then one has

$$
c=\frac{3}{2} g^{2} U_{0}^{4} .
$$

By substitution of eqs. (7.25) and (7.24) into eq. (7.23) one finds the cosmological time of YM condensate decay, approximately,

$$
\Delta t \simeq \sqrt{\frac{\kappa}{8}} \frac{\alpha^{2}}{g}, \quad \alpha \sim 10 .
$$

This provides us with the typical time scale for the YM condensate decay into particles which can be considered as an additional mechanism for particles production in the early Universe. An extension of cosmological dynamics of the YM system "condensate + waves" to an arbitrary YM theory accounting for higher order effects in wave modes will be done elsewhere.

\section{An overview of the two-condensate SU(4) model}

Let us consider a more complicated YM theory based on a large gauge group containing a few $\mathrm{SU}(2)$ subgroups. The simplest group of this type is $\mathrm{SU}(4)$. It contains two $\mathrm{SU}(2)$ gauge subgroups both isomorphic to the $\mathrm{SO}(3)$ spatial symmetry group which means that the YM field, described by local SU(4) gauge theory, contains two condensates. The main focus of this section is to discuss dynamical features of such a heterogenic vacuum system.

The generators of SU(4) gauge group can be written as

$$
\begin{aligned}
& \lambda^{1}=\left(\begin{array}{cccc}
0 & 1 & 0 & 0 \\
1 & 0 & 0 & 0 \\
0 & 0 & 0 & 0 \\
0 & 0 & 0 & 0
\end{array}\right), \quad \lambda^{2}=\left(\begin{array}{cccc}
0 & -i & 0 & 0 \\
i & 0 & 0 & 0 \\
0 & 0 & 0 & 0 \\
0 & 0 & 0 & 0
\end{array}\right), \quad \lambda^{3}=\left(\begin{array}{cccc}
1 & 0 & 0 & 0 \\
0 & -1 & 0 & 0 \\
0 & 0 & 0 & 0 \\
0 & 0 & 0 & 0
\end{array}\right), \\
& \lambda^{4}=\left(\begin{array}{cccc}
0 & 0 & 0 & 0 \\
0 & 0 & 0 & 0 \\
0 & 0 & 0 & 1 \\
0 & 0 & 1 & 0
\end{array}\right) \quad \lambda^{5}=\left(\begin{array}{cccc}
0 & 0 & 0 & 0 \\
0 & 0 & 0 & 0 \\
0 & 0 & 0 & -i \\
0 & 0 & i & 0
\end{array}\right), \quad \lambda^{6}=\left(\begin{array}{cccc}
0 & 0 & 0 & 0 \\
0 & 0 & 0 & 0 \\
0 & 0 & 1 & 0 \\
0 & 0 & 0 & -1
\end{array}\right) \text {, } \\
& \lambda^{7}=\left(\begin{array}{cccc}
0 & 0 & 1 & 0 \\
0 & 0 & 0 & 0 \\
1 & 0 & 0 & 0 \\
0 & 0 & 0 & 0
\end{array}\right), \quad \lambda^{8}=\left(\begin{array}{cccc}
0 & 0 & -i & 0 \\
0 & 0 & 0 & 0 \\
i & 0 & 0 & 0 \\
0 & 0 & 0 & 0
\end{array}\right) \quad \lambda^{9}=\left(\begin{array}{cccc}
0 & 0 & 0 & 0 \\
0 & 0 & 1 & 0 \\
0 & 1 & 0 & 0 \\
0 & 0 & 0 & 0
\end{array}\right) \text {, }
\end{aligned}
$$




$$
\begin{aligned}
& \lambda^{10}=\left(\begin{array}{cccc}
0 & 0 & 0 & 0 \\
0 & 0 & -i & 0 \\
0 & i & 0 & 0 \\
0 & 0 & 0 & 0
\end{array}\right), \quad \lambda^{11}=\frac{1}{\sqrt{2}}\left(\begin{array}{cccc}
1 & 0 & 0 & 0 \\
0 & 1 & 0 & 0 \\
0 & 0 & -1 & 0 \\
0 & 0 & 0 & -1
\end{array}\right), \quad \lambda^{12}=\left(\begin{array}{llll}
0 & 0 & 0 & 1 \\
0 & 0 & 0 & 0 \\
0 & 0 & 0 & 0 \\
1 & 0 & 0 & 0
\end{array}\right) \\
& \lambda^{13}=\left(\begin{array}{cccc}
0 & 0 & 0 & -i \\
0 & 0 & 0 & 0 \\
0 & 0 & 0 & 0 \\
i & 0 & 0 & 0
\end{array}\right), \quad \lambda^{14}=\left(\begin{array}{cccc}
0 & 0 & 0 & 0 \\
0 & 0 & 0 & 1 \\
0 & 0 & 0 & 0 \\
0 & 1 & 0 & 0
\end{array}\right), \quad \lambda^{15}=\left(\begin{array}{cccc}
0 & 0 & 0 & 0 \\
0 & 0 & 0 & -i \\
0 & 0 & 0 & 0 \\
0 & i & 0 & 0
\end{array}\right)
\end{aligned}
$$

The generators $\lambda^{1}, \lambda^{2}, \lambda^{3}$ and $\lambda^{4}, \lambda^{5}, \lambda^{6}$ correspond to $\mathrm{SU}(2)$ subgroups, and structure constants are given by

$$
f_{a b c}=\frac{1}{4 i} \operatorname{Tr}\left(\left[\lambda_{a}, \lambda_{b}\right] \lambda_{c}\right) .
$$

In the Hamilton gauge, two different YM condensates $U_{i}=U_{i}(t), i=1,2$ corresponding to each $\mathrm{SU}(2)$ subgroup can be introduced in analogy with eq. (2.8), i.e.

$$
A_{i}^{a}=\delta_{1 i}^{a} U_{1}+\delta_{2 i}^{a} U_{2}+\widetilde{A}_{i}^{a}, \quad a=1 \ldots 15,
$$

where

$$
\delta_{1 i}^{a}=\left(\begin{array}{ccc}
1 & 0 & 0 \\
0 & 1 & 0 \\
0 & 0 & 1 \\
0 & 0 & 0 \\
0 & 0 & 0 \\
0 & 0 & 0 \\
. & . & . \\
0 & 0 & 0
\end{array}\right), \quad \delta_{2 i}^{a}=\left(\begin{array}{ccc}
0 & 0 & 0 \\
0 & 0 & 0 \\
0 & 0 & 0 \\
1 & 0 & 0 \\
0 & 1 & 0 \\
0 & 0 & 1 \\
. & . & . \\
0 & 0 & 0
\end{array}\right) .
$$

The equations of motion are given by general formula from the classical YM theory (2.4). The equations for free (non-interacting) condensates $U_{1}$ and $U_{2}$ can be easily extracted from eq. (2.4)

$$
\partial_{0} \partial_{0} U_{1}+2 g^{2} U_{1}^{3}=0, \quad \partial_{0} \partial_{0} U_{2}+2 g^{2} U_{2}^{3}=0 .
$$

Note, these equations do not contain mixed terms like $U_{1}^{n} U_{2}^{m}$ and coincide with eq. (4.1). This means that the condensates $U_{1}(t)$ and $U_{2}(t)$ in the SU(4) theory do not interact with each other directly. As we will demonstrate later, they can interact only by means of particle exchanges.

The linear equations of motion for the Fourier transformed wave modes $\widetilde{A}_{i}^{\vec{p} a}$ (we omit index $\vec{p}$ below) are

$$
\begin{aligned}
\partial_{0} \partial_{0} \widetilde{A}_{k}^{a}+p^{2} \widetilde{A}_{k}^{a}-p^{2} n_{k} n_{i} \widetilde{A}_{i}^{a}+2 i g p U_{1} f_{a i c} n_{i} \widetilde{A}_{k}^{c}+2 i g p U_{2} f_{a(i+3) c} n_{i} \widetilde{A}_{k}^{c} \\
\quad+i g p U_{1} f_{a b k} n_{i} \widetilde{A}_{i}^{b}+i g p U_{2} f_{a b(k+3)} n_{i} \widetilde{A}_{i}^{b}+i g p U_{1} f_{a b i} n_{k} \widetilde{A}_{i}^{b}+i g p U_{2} f_{a b(i+3)} n_{k} \widetilde{A}_{i}^{b}=0 .
\end{aligned}
$$

One can show by a direct calculation that equations for the wave modes corresponding to each of the SU(2) subgroups (with $a=1,2,3$ and $a=4,5,6$, respectively) coincide with 
analogical equations in the one-condensate model (4.7) (or with eqs. (4.13) and (4.14) in terms of the tensor basis modes).

Now let us investigate the quasi-linear "back reaction" effect of the wave modes to YM condensates. Including next (second) order in waves, the equation for the $U_{1}$ condensate reads

$$
\begin{gathered}
\partial_{0} \partial_{0} U_{1}+2 g^{2} U_{1}^{3}+\frac{i g}{12} \sum_{\vec{p}} p f_{k b c}\left\langle 2 n_{i} \widetilde{A}_{i}^{b \dagger} \widetilde{A}_{k}^{c}-2 n_{i} \widetilde{A}_{i}^{b} \widetilde{A}_{k}^{c \dagger}+n_{i} \widetilde{A}_{k}^{c \dagger} \widetilde{A}_{i}^{b}\right. \\
\left.-n_{i} \widetilde{A}_{k}^{c} \widetilde{A}_{i}^{b \dagger}+n_{k} \widetilde{A}_{i}^{b \dagger} \widetilde{A}_{i}^{c}-n_{k} \widetilde{A}_{i}^{b} \widetilde{A}_{i}^{c \dagger}\right\rangle+\frac{g^{2}}{12} U_{1} \sum_{\vec{p}}\left\langle f_{k b i} f_{b d e}\left(\widetilde{A}_{i}^{d} \widetilde{A}_{k}^{e \dagger}+\widetilde{A}_{i}^{d \dagger} \widetilde{A}_{k}^{e}\right)\right. \\
\left.+f_{k b c} f_{b d k}\left(\widetilde{A}_{i}^{d} \widetilde{A}_{i}^{c \dagger}+\widetilde{A}_{i}^{d \dagger} \widetilde{A}_{i}^{c}\right)+f_{k b c} f_{b i e}\left(\widetilde{A}_{k}^{e} \widetilde{A}_{i}^{c \dagger}+\widetilde{A}_{k}^{e \dagger} \widetilde{A}_{i}^{c}\right)\right\rangle \\
+\frac{g^{2}}{12} U_{2} \sum_{\vec{p}}\left\langle f_{k b(i+3)} f_{b d e}\left(\widetilde{A}_{i}^{d} \widetilde{A}_{k}^{e \dagger}+\widetilde{A}_{i}^{d \dagger} \widetilde{A}_{k}^{e}\right)+f_{k b c} f_{b d(k+3)}\left(\widetilde{A}_{i}^{d} \widetilde{A}_{i}^{c \dagger}+\widetilde{A}_{i}^{d \dagger} \widetilde{A}_{i}^{c}\right)\right. \\
\left.+f_{k b c} f_{b(i+3) e}\left(\widetilde{A}_{k}^{e} \widetilde{A}_{i}^{c \dagger}+\widetilde{A}_{k}^{e \dagger} \widetilde{A}_{i}^{c}\right)\right\rangle=0 .
\end{gathered}
$$

The corresponding equation for the second condensate $U_{2}$ has an analogical form. By a direct calculation it can be shown from eq. (8.6), analogical equation for $U_{2}$ and eq. (8.5) that the wave modes corresponding to the first and second $\mathrm{SU}(2)$ subgroup interact only with its own condensate $U_{1}$ and $U_{2}$, respectively, in the same way as they do in the onecondensate model considered above. Remarkably enough, other 27 modes corresponding $a=7 \ldots 15$ generators of $\mathrm{SU}(4)$ interact with both condensates at the same time. This means that interaction between $U_{1}$ and $U_{2}$ condensates is realised via particle exchanges only related to these remaining 27 wave modes. This effect is explicitly confirmed by a numerical analysis.

In general, time evolution of wave modes of the SU(4) theory and two YM condensates is analogical to the case of one-condensate system illustrated in figure 3, i.e. energy of the both condensates is transferred into the ultra-relativistic YM plasma. This study suggests that the observed effect of energy swap is a generic feature of YM dynamics.

\section{Summary}

Starting from the basic idea about an important dynamical role of the YM condensate (2.8), we have constructed a consistent quasi-classical approach based on Hamilton formulation and canonical quantisation of the wave modes in the classical YM condensate. This approach has been applied in numerical analysis of the system of YM wave modes (or particles after quantisation) in the ultra-relativistic gluon plasma interacting with the YM condensate (in the limit of small interactions between the wave modes) as well as in analytic investigation of generic YM "condensate + waves" dynamics with all the interaction terms included.

First, the quantum energy spectrum of free (no waves) YM condensate has been found from the stationary Schrödinger equation. It turned out that this spectrum corresponds 
to a potential well of the fourth power, and a convenient analytical approximation to the discrete numerical solution has been proposed.

Next, we have derived the YM equations of motion for the "condensate + waves" system in linear approximation (4.1), (4.13) and (4.14) in the $\mathrm{SU}(2)$ gauge theory and numerically investigated their solutions. As an important effect which removes degeneracy in the underlined gauge theory the interactions of wave modes with the YM condensate lead to dynamical generation of the longitudinal d.o.f.'s in the gluon plasma.

Also, in order to understand how condensate-waves interactions affect the energy balance between these two YM subsystems, we have investigated their dynamics in quasi-linear and next-to-linear approximations accounting for the "back reaction" effect of particles to the condensate (4.25). The higher order terms have also been studied and found to be important for late-time stability of the YM solutions. The results of numerical analysis are presented in figures 3 and 4 and demonstrate a characteristic energy swap effect, namely, an effective energy transfer from initially large condensate fluctuations to the YM wave modes. This effect of condensate decay can thus be considered as a possible source for heating up the ultra-relativistic gluon plasma as well as potentially gives rise to particle production mechanisms in the hot cosmological plasma in the early Universe.

At the next step, the equations for pure gluon plasma (without the YM condensate) in the $\mathrm{SU}(3)$ gauge theory have been derived in different approximations. The thirdorder interaction terms in waves dynamically induce a local constituent gluon mass. The corresponding renormalized expression appears to be consistent with thermodynamic predictions. The second-order interactions initiate the dynamical gluon mass terms in a time non-local form. In addition, the exact integro-differential equations for the condensate and waves evolution incorporating all the higher-order interaction terms have been derived for the $\mathrm{SU}(2)$ theory.

It has been shown that dynamics of waves and condensate in the extended $\mathcal{N}=4$ supersymmetric YM theory is analogical to the one of pure YM theory discussed above. In particular, it has been indicated that interaction of the supersymmetric (pseudo)scalar wave modes with the YM condensate leads to a similar energy swap effect between them. A similar energy swap effect has also been found in the heterogenic system of two interacting YM condensates in the SU(4) gauge theory. These findings strongly suggest that the observed dynamics in energy balance of the interacting YM "wave + condensate" system is a general phenomenon and a specific property inherent to a variety of YM theories with condensate.

We also considered conformal time dynamics of free non-interacting YM condensate in the Friedmann Universe. The non-linear oscillations of the condensate behaving as radiation matter in the course of Universe expansion appears to be unstable w.r.t. quantum corrections. In order to address the latter issue the leading vacuum polarisation effects at one-loop have been incorporated. Such a quasi-free condensate contribution to the energy density dramatically changes its behavior to a positive constant in time. This behaviour is consistent with the vacuum equation of state and thus can be used to compensate large negative topological vacua terms [17]. The latter would potentially offer a consistent way to address an observable smallness of the cosmological constant but a self-consistent analysis 
of the waves with the vacuum polarisation effects will be critical for that. As a toy-model void of radiative corrections, the Super-Yang-Mills theory with condensate interacting with small waves has been considered in the Friedmann Universe and the condensate decay time has been estimated in quasi-linear approximation. The latter approach once applied to a realistic interacting gauge theory could provide an access to typical time scale for particle production, in particular, during the QCD transition epoch and/or in the preheating stage of a gauge-field driven inflation in Cosmology. These important aspects will be discussed elsewhere.

Finally, we have investigated the gauge dependence of the YM condensate and waves. It turned out that it is possible to establish a connection between the results in the Hamilton gauge and those in arbitrary gauge by the method of absorbing the gauge dependence into a coordinate transformation. In this framework the YM condensate in arbitrary gauge can be considered as a functional of the complete YM solution in the Hamilton gauge and the gauge-fixing function. Our thorough analysis of non-degenerate YM dynamics performed in the Hamilton gauge has appeared to be justified as the most straightforward and convenient since these results can be then, in principle, transformed into those in any given gauge.

To summarize, as one of the main results of this paper, the energy redistribution effect from the YM condensate to the YM wave modes in the case of initially small wave amplitudes has been found and investigated from the first principles of canonical quasiclassical YM theory in different settings and approximations. As was mentioned above, this effect can be of major importance for cosmological processes in the early Universe, in particular, in the processes of particle production during the preheating period after cosmic inflation which is planned for further studies. In addition, an extension of the quasi-classical approach to a full quantum field theory formalism (including fermion modes and vacuum polarisation effects) could become one of the next important steps in further theoretical understanding of interacting YM dynamics of "condensate + waves" systems.

\section{A The method of infinitesimal parameter}

The major difficulties of the canonical quantization of a free YM field (without the YM condensate) arise due to the presence of its time-retarded zero component in the Lagrangian (2.1). One of the ways to resolve this issue is based upon the method of asymptotic expansion in the configuration space elaborated in refs. $[32,33]$. Here, we advise an alternative method of infinitesimal parameter which is also applicable in the Hamilton gauge (2.3).

Let us construct the YM propagator in the form of chronologically ordered operator product averaged over the state vector. Consider a YM system containing a YM field $A_{\mu}^{a}$ and a charged multiplet of fermions interacting with $A_{\mu}^{a}$. The Lagrangian and Hamiltonian densities of such a system are

$$
\begin{aligned}
\mathcal{L} & =-\frac{1}{4} F_{\mu \nu}^{a} F_{a}^{\mu \nu}+i \bar{q}_{f} \gamma^{\mu}\left(\partial_{\mu} q_{f}-\frac{i}{2} g A_{\mu}^{a} \lambda^{a} q_{f}\right)-m_{f} \bar{q}_{f} q_{f}, \\
\mathcal{H} & =\frac{1}{2}\left(\left(F_{0 k}^{a}\right)^{2}+\left(F_{i k}^{a}\right)^{2}\right)-\frac{1}{2} g \bar{q}_{f} \gamma^{i} A_{i}^{a} \lambda^{a} q_{f}-i \bar{q}_{f} \gamma^{i} \partial_{i} q_{f}+m_{f} \bar{q}_{f} q_{f},
\end{aligned}
$$


respectively. Then, the corresponding Lagrange equations of motion read

$$
\begin{aligned}
\partial^{\mu} F_{\mu \nu}^{a}-g F_{\mu \nu}^{b} f_{a b c} A_{c}^{\mu}+\frac{1}{2} g \bar{q}_{f} \gamma_{\nu} \lambda^{a} q_{f} & =0, \\
i \gamma^{\mu} \partial_{\mu} q_{f}+\frac{1}{2} g \gamma^{\mu} A_{\mu}^{a} \lambda^{a} q_{f}-m_{f} q_{f} & =0, \\
-i \partial_{\mu} \bar{q}_{f} \gamma^{\mu}+\frac{1}{2} g \bar{q}_{f} \gamma^{\mu} A_{\mu}^{a} \lambda^{a}-m_{f} \bar{q}_{f} & =0 .
\end{aligned}
$$

The canonical quantization procedure is based upon the (anti)commutation relations between the field operators

$$
\begin{aligned}
& {\left[E_{i}^{a}(t, \vec{x}), A_{k}^{b}\left(t, \vec{x}^{\prime}\right)\right]_{-}=-i \delta_{i k} \delta_{a b} \delta\left(\vec{x}-\vec{x}^{\prime}\right),} \\
& {\left[q_{f}^{\alpha}(t, \vec{x}), P_{f^{\prime}}^{\beta}\left(t, \vec{x}^{\prime}\right)\right]_{+}=i \delta_{\alpha \beta} \delta_{f f^{\prime}} \delta\left(\vec{x}-\vec{x}^{\prime}\right),}
\end{aligned}
$$

where generalized momenta conjugated to the fields $A_{k}^{a}$ and $q_{f}^{\alpha}$ are found as

$$
\begin{aligned}
& E_{k}^{a}=\frac{\delta}{\delta\left(\partial_{0} A_{k}^{a}\right)} \int \mathcal{L} d^{3} x=F_{0 k}^{a}, \\
& P_{f}^{\alpha}=\frac{\delta}{\delta\left(\partial_{0} q_{f}^{\alpha}\right)} \int \mathcal{L} d^{3} x=i \bar{q}_{f}^{\alpha} \gamma^{0},
\end{aligned}
$$

respectively. Here, we kept color index $\alpha$ of a quark flavor $f$ for transparency, while it is often omitted in other places. The system of quantum equations of motion can be written in the Heisenberg representation

$$
\begin{array}{r}
\partial_{0} F_{0 k}^{a}=\partial_{i} F_{i k}^{a}-g F_{i k}^{b} f_{a b c} A_{i}^{c}-\frac{1}{2} g \bar{q}_{f} \gamma_{k} \lambda^{a} q_{f}, \\
i \gamma^{\mu} \partial_{\mu} q_{f}+\frac{1}{2} g \gamma^{i} A_{i}^{a} \lambda^{a} q_{f}-m_{f} q_{f}=0, \\
-i \partial_{\mu} \bar{q}_{f} \gamma^{\mu}+\frac{1}{2} g \bar{q}_{f} \gamma^{i} A_{i}^{a} \lambda^{a}-m_{f} \bar{q}_{f}=0, \\
\partial^{0}\left(\partial^{i} F_{i 0}^{a}-g f_{a b c} F_{i 0}^{b} A_{c}^{i}+\frac{1}{2} g \bar{q}_{f} \gamma_{0} \lambda^{a} q_{f}\right)=0,
\end{array}
$$

as well as in the interaction representation

$$
\partial_{0} \partial_{0} A_{i}^{a}-\partial_{k} \partial_{k} A_{i}^{a}+\partial_{i} \partial_{k} A_{k}^{a}=0, \quad i \gamma^{\mu} \partial_{\mu} q_{f}-m_{f} q_{f}=0, \quad i \partial_{\mu} \bar{q}_{f} \gamma^{\mu}+m_{f} \bar{q}_{f}=0 .
$$

Performing the Fourier transformation, one arrives at the system of equations for the longitudinal $A_{\vec{p} 0}^{a}$ and transverse components $A_{\vec{p} \lambda}^{a}, \lambda=1,2$ of the YM field in the following form

$$
\partial_{0} \partial_{0} A_{\vec{p} 0}^{a}=0, \quad \partial_{0} \partial_{0} A_{\vec{p} \lambda}^{a}+|\vec{p}|^{2} A_{\vec{p} \lambda}^{a}=0,
$$

respectively. The equation for the longitudinal mode does not have a wave solution, so it is impossible to take into account its contribution in the YM propagator constructed as vacuum average of the chronologically ordered operator product. 
In order to resolve this problem we can modify the Hamiltonian density by means of adding an extra small "virtual" term depending on an infinitesimal parameter $\zeta \ll 1$ and vanishing at $\zeta \rightarrow 0$, i.e.

$$
\mathcal{H}_{\text {mod }}=\int\left[\frac{1}{2}\left(\partial_{0} A_{k}^{a} \partial_{0} A_{k}^{a}+\partial_{l} A_{k}^{a} \partial_{l} A_{k}^{a}-\left(1-\zeta^{2}\right) \partial_{l} A_{k}^{a} \partial_{k} A_{l}^{a}\right)-i \bar{q}_{f} \gamma^{i} \partial_{i} q_{f}+m_{f} \bar{q}_{f} q_{f}\right] d^{3} x .
$$

Due to such a modification, the equation of motion for the longitudinal component becomes

$$
\partial_{0} \partial_{0} A_{\vec{p} 0}^{a}+\zeta^{2}|\vec{p}|^{2} A_{\vec{p} 0}^{a}=0
$$

such that it acquires an infinitesimal frequency. This modified equation enables us to incorporate the longitudinal mode into the YM propagator which is given by (in the limit $\zeta \rightarrow 0$ )

$$
D_{i k}^{a b}\left(x-x^{\prime}\right)=\frac{\delta_{a b}}{(2 \pi)^{4}} \int\left(\delta_{i k}-\frac{p_{i} p_{k}}{p_{0}^{2}+i \epsilon}\right) \frac{d^{4} p}{p^{2}-p_{0}^{2}-i \epsilon} e^{-i p\left(x-x^{\prime}\right)},
$$

where $p_{0}$ is the zeroth component of the YM field momentum $p$. Then the fermion propagator takes the standard form, i.e.

$$
D_{f f^{\prime}}\left(x-x^{\prime}\right)=\frac{\delta_{f f^{\prime}}}{(2 \pi)^{4}} \int\left(\gamma_{\mu} p^{\mu}+m_{f}\right) \frac{d^{4} p}{p^{2}-m_{f}^{2}+i \epsilon} e^{-i p\left(x-x^{\prime}\right)} .
$$

Note, the formulae (A.6) and (A.7) coincide with the corresponding Green functions constructed for the initial (non-modified) YM equations of motion (A.3), thus, validating the proposed method.

\section{B Canonical quantization of the Yang-Mills wave modes}

Let us now perform canonical quantisation of the YM wave modes in the classical YM condensate and therefore construct the quasi-classical YM theory. For this purpose, as the matter of the Bohr's correspondence principle we introduce operators instead of field functions in the Hamiltonian density of, for example, the $\mathcal{N}=4$ supersymmetric YM theory (6.3). Then we impose (anti)commutation relations to the field operators for each wave mode as follows

$$
\begin{aligned}
P_{\Phi} & =\frac{1}{2} \partial_{0} \Phi^{\dagger}, & {\left[P_{\Phi}, \Phi\right]_{-} } & =-i, \\
P_{\Lambda} & =\frac{1}{4} \partial_{0} \Lambda^{\dagger}, & {\left[P_{\Lambda}, \Lambda\right]_{-} } & =-i, \\
P_{\lambda} & =\frac{1}{2} \partial_{0} \lambda^{\dagger}, & {\left[P_{\lambda}, \lambda\right]_{-} } & =-i, \\
P_{\phi}^{\sigma} & =\frac{1}{2} \partial_{0} \phi_{\sigma}^{\dagger}, & {\left[P_{\phi}^{\sigma}, \phi_{\gamma}\right]_{-} } & =-i \delta_{\sigma \gamma}, \\
P_{\eta}^{\sigma} & =\frac{1}{2} \partial_{0} \eta_{\sigma}^{\dagger}, & {\left[P_{\eta}^{\sigma}, \eta_{\gamma}\right]_{-} } & =-i \delta_{\sigma \gamma}, \\
P_{\psi}^{\sigma} & =\frac{1}{2} \partial_{0} \psi_{\sigma}^{\dagger}, & {\left[P_{\psi}^{\sigma}, \psi_{\gamma}\right]_{-} } & =-i \delta_{\sigma \gamma}, \\
P_{K}^{(i)} & =\frac{1}{4} \partial_{0} K_{(i)}^{\dagger}, & {\left[P_{K}^{(i)}, K_{(j)}\right]_{-} } & =-i \delta_{i j},
\end{aligned}
$$




$$
\begin{aligned}
P_{M}^{(i) \alpha} & =\frac{1}{4} \partial_{0} M_{(i) \alpha}^{\dagger}, & {\left[P_{M}^{(i) \alpha}, M_{(j) \beta}\right]_{-} } & =-i \delta_{i j} \delta_{\alpha \beta}, \\
P_{\vartheta}^{k^{\prime}} & =\frac{i}{4} \bar{\vartheta}^{k^{\prime}} \gamma_{0}, & {\left[\vartheta^{l^{\prime}}, P_{\vartheta}^{k^{\prime}}\right]_{+} } & =i \delta_{l^{\prime} k^{\prime}}, \\
P_{\zeta}^{k^{\prime} \alpha} & =\frac{i}{4} \bar{\zeta}_{\alpha}^{k^{\prime}} \gamma_{0}, & {\left[\zeta_{\alpha}^{l^{\prime}}, P_{\zeta}^{k^{\prime} \beta}\right]_{+} } & =i \delta_{l^{\prime} k^{\prime}} \delta_{\alpha \beta} .
\end{aligned}
$$

Commutation relations for pseudoscalar modes $K^{\prime}$ and $M^{\prime}$ are the same as for scalar ones $K$ and $M$, respectively. In addition, analogical formulas for Hermitian conjugate modes $\Phi^{\dagger}, \Lambda^{\dagger}$, etc should be added.

Finally, quantum Hamilton equations in commutators can be constructed in the standard way. For example, for the $\Phi$ mode we have

$$
\partial_{0} \Phi=i[\mathcal{H}, \Phi]_{-}, \quad \partial_{0} P_{\Phi}=i\left[\mathcal{H}, P_{\Phi}\right]_{-} .
$$

Such equations written for all wave modes coincide with the corresponding equations of motion which were constructed previously (4.13), (4.14) and (6.4)-(6.7). The latter is an important validation test of our calculations.

\section{Acknowledgments}

This work was supported in part by the Crafoord Foundation (Grant No. 20120520). R. P. is grateful to the "Beyond the LHC" Program at Nordita (Stockholm) for support and hospitality during completion of this work.

Open Access. This article is distributed under the terms of the Creative Commons Attribution License (CC-BY 4.0), which permits any use, distribution and reproduction in any medium, provided the original author(s) and source are credited.

\section{References}

[1] A.A. Belavin, A.M. Polyakov, A.S. Schwartz and Y. Tyupkin, Pseudoparticle solutions of the Yang-Mills equations, Phys. Lett. B 59 (1975) 85 [INSPIRE].

[2] T. Schäfer and E.V. Shuryak, Instantons in QCD, Rev. Mod. Phys. 70 (1998) 323 [hep-ph/9610451] [INSPIRE].

[3] V. Gogohia and H. Toki, Topological structure of chiral QCD vacuum, Phys. Rev. D 61 (2000) 036006 [Erratum ibid. D 63 (2001) 079901] [hep-ph/9908301] [INSPIRE].

[4] V. Gogohia and G. Kluge, Determination of the quantum part of the truly nonperturbative Yang-Mills vacuum energy density in the covariant gauge $Q C D$, Phys. Rev. D 62 (2000) 076008 [Erratum ibid. D 63 (2001) 099902] [hep-ph/0002003] [INSPIRE].

[5] J.-P. Blaizot, F. Gelis, J.-F. Liao, L. McLerran and R. Venugopalan, Bose-Einstein condensation and thermalization of the quark gluon plasma, Nucl. Phys. A 873 (2012) 68 [arXiv:1107.5296] [inSPIRE]. 
[6] J.-P. Blaizot, J. Liao and L. McLerran, Gluon transport equation in the small angle approximation and the onset of Bose-Einstein condensation, Nucl. Phys. A 920 (2013) 58 [arXiv: 1305.2119] [INSPIRE].

[7] X.-G. Huang and J. Liao, Glasma evolution and Bose-Einstein condensation with elastic and inelastic collisions, arXiv:1303.7214 [INSPIRE].

[8] D.V. Gal'tsov and E.A. Davydov, Yang-Mills condensates in cosmology, Int. J. Mod. Phys. Conf. Ser. 14 (2012) 316 [arXiv:1112.2943] [INSPIRE].

[9] D.V. Galtsov and M.S. Volkov, Yang-Mills cosmology: cold matter for a hot universe, Phys. Lett. B 256 (1991) 17 [INSPIRE].

[10] V.V. Dyadichev, D.V. Gal'tsov, A.G. Zorin and M.Y. Zotov, Non-Abelian Born-Infeld cosmology, Phys. Rev. D 65 (2002) 084007 [hep-th/0111099] [INSPIRE].

[11] D.V. Gal'tsov and E.A. Davydov, Cosmological models with gauge fields, Proc. Steklov Inst. Math. 272 (2011) 119 [arXiv: 1012.2861] [INSPIRE].

[12] E. Elizalde, A.J. Lopez-Revelles, S.D. Odintsov and S.Y. Vernov, Cosmological models with Yang-Mills fields, Phys. Atom. Nucl. 76 (2013) 996 [arXiv:1201.4302] [InSPIRE].

[13] A. Maleknejad and M.M. Sheikh-Jabbari, Gauge-flation: inflation from non-Abelian gauge fields, Phys. Lett. B 723 (2013) 224 [arXiv:1102.1513] [INSPIRE].

[14] A. Maleknejad and M.M. Sheikh-Jabbari, Non-Abelian gauge field inflation, Phys. Rev. D 84 (2011) 043515 [arXiv:1102.1932] [INSPIRE].

[15] F.R. Urban and A.R. Zhitnitsky, The QCD nature of dark energy, Nucl. Phys. B 835 (2010) 135 [arXiv:0909.2684] [INSPIRE].

[16] R. Pasechnik, V. Beylin and G. Vereshkov, Dark energy from graviton-mediated interactions in the QCD vacuum, JCAP 06 (2013) 011 [arXiv: 1302.6456] [INSPIRE].

[17] R. Pasechnik, V. Beylin and G. Vereshkov, Possible compensation of the QCD vacuum contribution to the dark energy, Phys. Rev. D 88 (2013) 023509 [arXiv:1302.5934] [INSPIRE].

[18] L.D. Faddeev and A.A. Slavnov, Gauge fields. Introduction to quantum theory, $2^{\text {nd }}$ edition, Addison-Wesley, U.S.A. (1991).

[19] L.D. Faddeev and V.N. Popov, Feynman diagrams for the Yang-Mills field, Phys. Lett. B 25 (1967) 29 [InSPIRE].

[20] J.D. Jackson, Classical electrodynamics, $3^{\text {rd }}$ edition, Wiley, U.S.A. (1999).

[21] D.J. Griffiths, Introduction to electrodynamic, $3^{\text {rd }}$ edition, Prentice Hall, U.S.A. (1999).

[22] J.-P. Blaizot and E. Iancu, Non-Abelian plane waves in the quark-gluon plasma, Phys. Lett. B 326 (1994) 138 [hep-ph/9401323] [INSPIRE].

[23] V.M. Bannur, Relativistic longitudinal nonAbelian oscillations in quark anti-quark plasma, Pramana 59 (2002) 671.

[24] B. Schenke, Collective phenomena in the non-equilibrium quark-gluon plasma, arXiv:0810.4306 [INSPIRE].

[25] F. Gliozzi, J. Scherk and D.I. Olive, Supersymmetry, supergravity theories and the dual spinor model, Nucl. Phys. B 122 (1977) 253 [INSPIRE].

[26] N.N. Bogoliubov and D.V. Shirkov, Quantum fields, Addison-Wesley, U.S.A. (1982). 
[27] L.D. Landau and E.M. Lifshitz, The classical theory of fields, Course of Theoretical Physics Series, $4^{\text {th }}$ edition volume 2, Butterworth-Heinemann, U.K. (1980).

[28] A. Kurkela and G.D. Moore, UV cascade in classical Yang-Mills theory, Phys. Rev. D 86 (2012) 056008 [arXiv:1207.1663] [InSPIRE].

[29] J. Berges, S. Schlichting and D. Sexty, Over-populated gauge fields on the lattice, Phys. Rev. D 86 (2012) 074006 [arXiv:1203.4646] [InSPIRE].

[30] J. Berges, K. Boguslavski, S. Schlichting and R. Venugopalan, Universal attractor in a highly occupied non-Abelian plasma, Phys. Rev. D 89 (2014) 114007 [arXiv:1311.3005] [INSPIRE].

[31] A. Hautot and A. Magnus, Calculation of the eigenvalues of Schrdinger equations by an extension of Hill's method, J. Comp. Appl. Math. 5 (1979) 3.

[32] I.A. Batalin and G.A. Vilkovisky, Gauge algebra and quantization, Phys. Lett. B 102 (1981) 27 [INSPIRE].

[33] I.A. Batalin and G.A. Vilkovisky, Quantization of gauge theories with linearly dependent generators, Phys. Rev. D 28 (1983) 2567 [Erratum ibid. D 30 (1984) 508] [INSPIRE].

[34] J. Cervero and L. Jacobs, Classical Yang-Mills fields in a Robertson-Walker universe, Phys. Lett. B 78 (1978) 427 [INSPIRE].

[35] Y. Hosotani, Exact solution to the Einstein Yang-Mills equation, Phys. Lett. B 147 (1984) 44 [INSPIRE].

[36] B.K. Darian and H.P. Kunzle, Cosmological Einstein Yang-Mills equations, J. Math. Phys. 38 (1997) 4696 [gr-qc/9610026] [INSPIRE].

[37] J.A.R. Cembranos, A.L. Maroto and S.J.N. Jareño, Isotropy theorem for cosmological Yang-Mills theories, Phys. Rev. D 87 (2013) 043523 [arXiv: 1212.3201] [INSPIRE].

[38] S.G. Matinyan and G.K. Savvidy, Vacuum polarization induced by the intense gauge field, Nucl. Phys. B 134 (1978) 539 [InSPIRE]. 